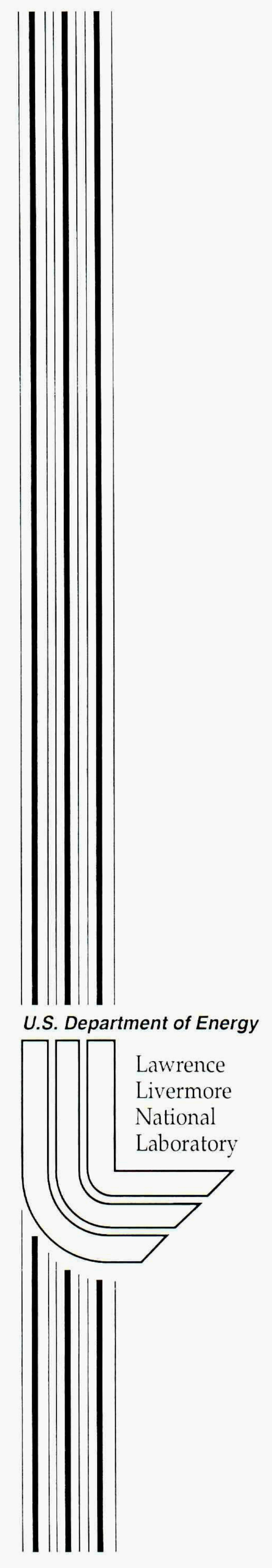

UCRL-ID-148081

\title{
Physics Advanced Technologies Directorate 2001 Annual Report
}

R. Jacobs

May 9, 2002 


\section{Physics and Advanced} Technologies

. 2001 Annual Report
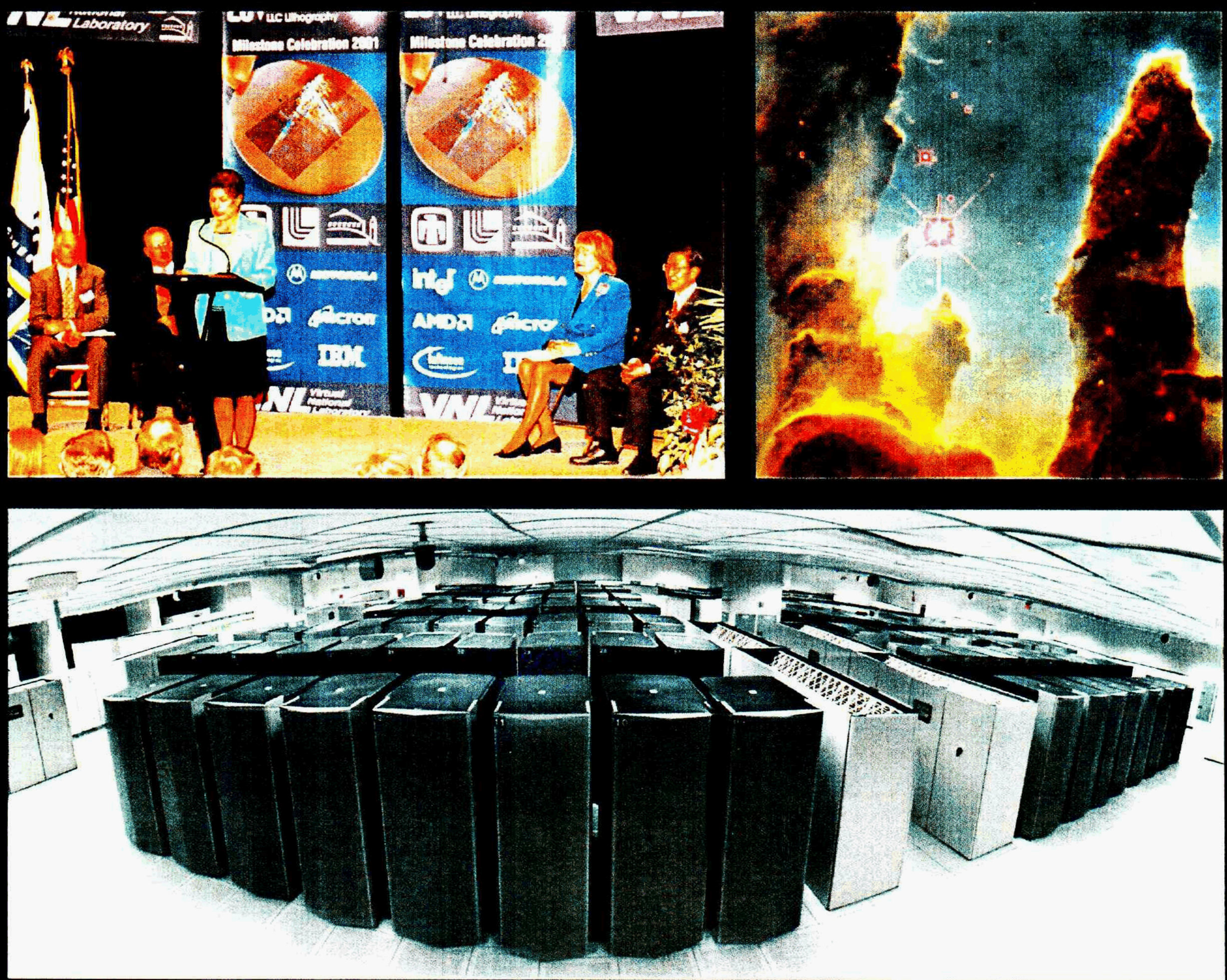

L4 


\section{Front cover}

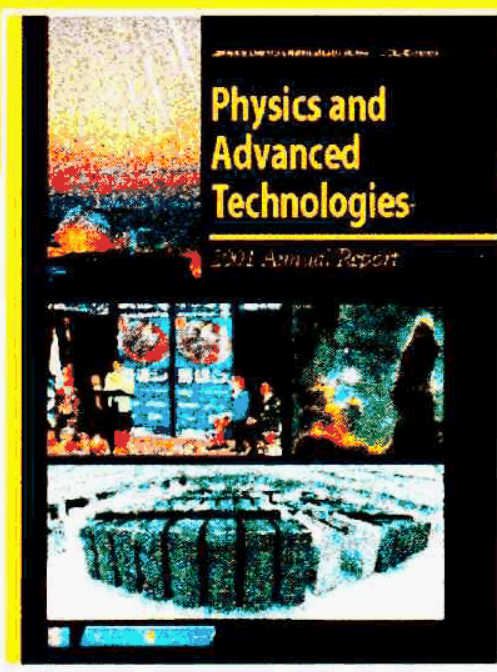

Top

Shown in this photo of the Keck "virtual" guide star is the orange laser beam emerging from the dome of the Keck II Telescope atop 14,000-foot Mauna Kea volcano in Hawaii. In this 20 -minute time exposure, motions of the stars show as streaks in the sky, and the hazard lights of the observatory appear in the road in the foreground. Physics and Advanced Technologies Directorate scientists helped install the guide star and are using it in a search for extra solar planets and other investigations. (Photo credit: John McDonald, Canada-France-Hawaii Telescope Facility.)

\section{Middle Left}

Dr. Miriam John of Sandia National Laboratories (SNL) welcomed researchers from the national laboratories and members of industry and government to the EUVL Milestone Celebration on April 11,2001. The Celebration marked the completion of the first full-scale prototype lithography machine for making computer chips using extreme ultraviolet light - a technology pioneered by PAT scientists and advanced in collaboration with industry, SNL, and Lawrence Berkeley National Laboratory.

\section{Middle Right}

Thanks to analysis done by PAT physicists, scientists may be closer to under standing the formation of the Eagle Nebula. Dramatic images of the Eagle Nebula and its towering Pillars of Creation, which reach almost 6 trillion miles high, were captured by the Hubble Space Telescope. (Photo credit: Jeff Hester and Paul Scowen and NASA.)

\section{Bottom}

From the U.S. Department of Energy's Advanced Simulation and Computing (ASCI), PAT researchers are using ASCI White, the world's largest and most powerful supercomputer, in Stockpile Stewardship projects. In addition to it use to simulate nuclear weapons tests, ASCI White will provide a platform for medical simulations, genetic computing, global climate modeling, aerospace and automotive design, and astrophysicist research, such as the Djehuty Project.

\section{Back cover}

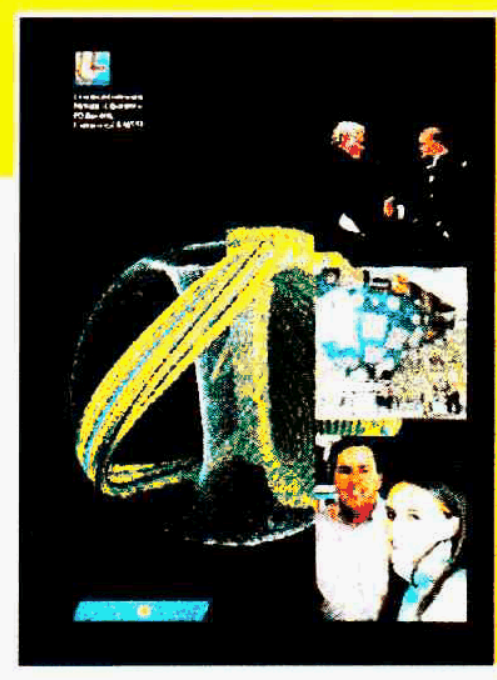

Top

Former LLNL physicist and current Stanford professor Robert B. Laughlin (left) is congratulated by Swedish King Carl XVI Gustaf as a co-winner of the 1998 Nobel Prize in Physics. Along with Horst L. Stoermer of Columbia University and Daniel C. Tsui of Princeton University, Laughlin was recognized for the discovery of a new form of "quantum fluid" that represents "yet another breakthrough in our understanding of quantum physics and to the development of new theoretical concepts of significance in many branches of modern physics." Laughlin's early work was begun and advanced while he was in the predecessor organization to the PAT Directorate. He is the first National Laboratory embloyee to win the Nobel Prize.

\section{Middle}

Shown is the National Ignition Facility target chamber where NIF's 192 laser beams will converge upon a fusion-fuelfilled target the size of a BB. PAT scientists will strongly participate in understanding NIF's high-energy-density and fusion regimes with direct applications to Stockpile Stewardship, energy research, science, and astrophysics.

\section{Middle Background}

This computer simulation from PAT's Fusion Energy Program, relevant to magnetic fusion energy research, shows a magnetic field line (white) wrapping around a torus, or doughnut-shaped configuration of plasma.

\section{Bottom}

Jenny Peyser, who suffers from Type-I diabetes, may benefit from the PAT-led glucose monitoring technology being developed by an award-winning research team that includes her father, physicist Tom Peyser. (Photo credit: Margaret Kaye.)

May 2002

UCRL-ID-148081
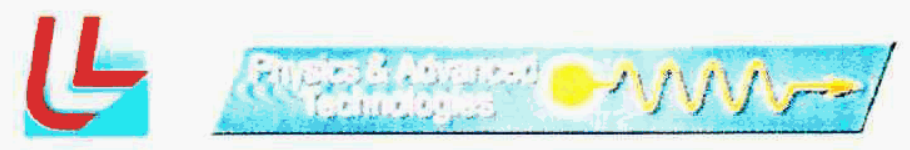

X1235

\section{(}

\author{
Produced by \\ Technical Editor \\ Ralph Jacobs \\ Publication Editor \\ Gloria Cannon \\ Design and Production \\ John Danielson \\ Jim McInnis \\ Compositors \\ Janet Tanaka \\ Sherry Emmons \\ Kathy O'Connor \\ Proofreaders \\ Emmeline Chen \\ Nona Sanford
}

\section{DISCLAIMER}

This document was prepared as an account of work sponsored by an agency of the United States Government. Neither the United States Government nor the University of California nor any of their employees, makes any warranty, express or implied, or assumes any legal liability or responsibility for the accuracy, completeness, or usefulness of any information, apparatus, product, or process disclosed, or represents that its use would not infringe privately owned rights. Reference herein to any specific commercial product, process, or service by trade name, trademark, manufacturer, or otherwise, does not necessarily constitute or imply its endorsement, recommendation, or favoring by the United States Government or the University of California. The views and opinions of authors expressed herein do not necessarily state or reflect those of the United States Government or the University of California, and shall not be used for advertising or product endorsement purposes.

This work was performed under the auspices of the U.S Department of Energy by University of California, Lawrence Livermore National Laboratory under Contract W-7405-Eng-48.

$$
\begin{aligned}
& \text { This report has been reproduced } \\
& \text { directly from the best available copy. }
\end{aligned}
$$

Available to DOE and DOE contractors from the Office of Scientific and Technical Information P.O. Box 62, Oak Ridge, TN 37831

Prices available from (615) 576-8401, FTS 626-8401 Available to the public from the National Technical Information Service

U.S. Department of Commerce 5285 Port Royal Rd. Springfield, VA 2216 


\section{DISCLAIMER}

This document was prepared as an account of work sponsored by an agency of the United States Government. Neither the United States Government nor the University of California nor any of their employees, makes any warranty, express or implied, or assumes any legal liability or responsibility for the accuracy, completeness, or usefulness of any information, apparatus, product, or process disclosed, or represents that its use would not infringe privately owned rights. Reference herein to any specific commercial product, process, or service by trade name, trademark, manufacturer, or otherwise, does not necessarily constitute or imply its endorsement, recommendation, or favoring by the United States Government or the University of California. The views and opinions of authors expressed herein do not necessarily state or reflect those of the United States Government or the University of California, and shall not be used for advertising or product endorsement purposes.

This work was performed under the auspices of the U. S. Department of Energy by the University of California, Lawrence Livermore National Laboratory under Contract No. W-7405-Eng-48.

This report has been reproduced directly from the best available copy.

Available electronically at http://www.doc.gov/bridge

Available for a processing fee to U.S. Department of Energy

And its contractors in paper from

U.S. Department of Energy

Office of Scientific and Technical Information

P.O. Box 62

Oak Ridge, TN 37831-0062

Telephone: (865) 576-8401

Facsimile: (865) 576-5728

E-mail: reports@adonis.osti.gov

Available for the sale to the public from

U.S. Department of Commerce

National Technical Information Service

5285 Port Royal Road

Springfield, VA 22161

Telephone: (800) 553-6847

Facsimile: (703) 605-6900

E-mail: orders@ntis.fedworld.gov

Online ordering: http://www.ntis.gov/ordering.htm

OR

Lawrence Livermore National Laboratory

Technical Information Department's Digital Library

http://www.llnl.gov/tid/Library.html 


\section{Physics and Advanced Technologies Directorate 2001 Annual Report Contents}

Introduction .1

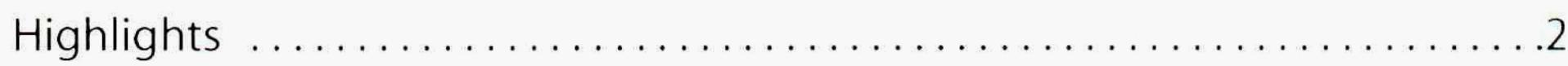

High-Energy-Density Physics and Astrophysics Division (V Division) $\ldots \ldots 6$ Condensed Matter Physics Division(H Division) $\ldots \ldots \ldots \ldots \ldots \ldots \ldots \ldots \ldots \ldots$

Nuclear, Particle, and Accelerator Physics Division (N Division) ..........16

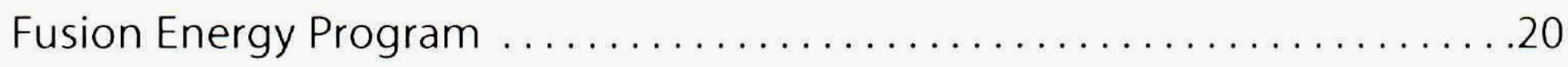

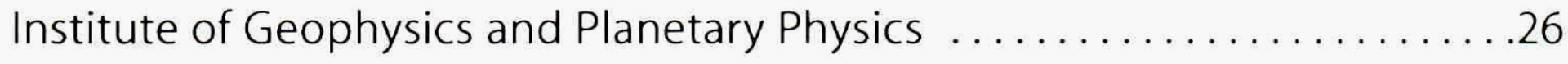

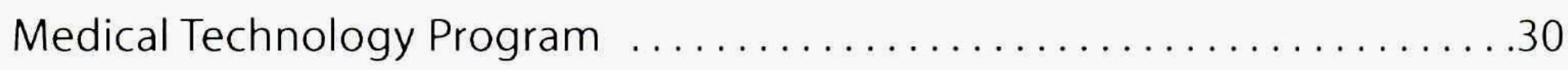

Extreme Ultraviolet Lithography Program $\ldots \ldots \ldots \ldots \ldots \ldots \ldots \ldots \ldots \ldots \ldots \ldots \ldots$

Imaging and Advanced Detectors Division (I Division) . .............40

Advanced Interceptor Technology Program $\ldots \ldots \ldots \ldots \ldots \ldots \ldots$

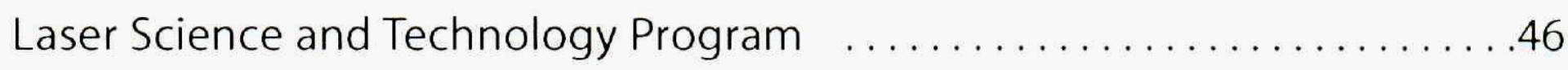

Institute for Laser Science and Applications ...................... 50

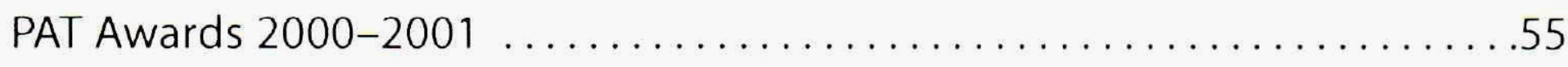

Research Retrospectives: Fusion Energy, Astrophysics, X-Ray Lasers . . . . . 64 

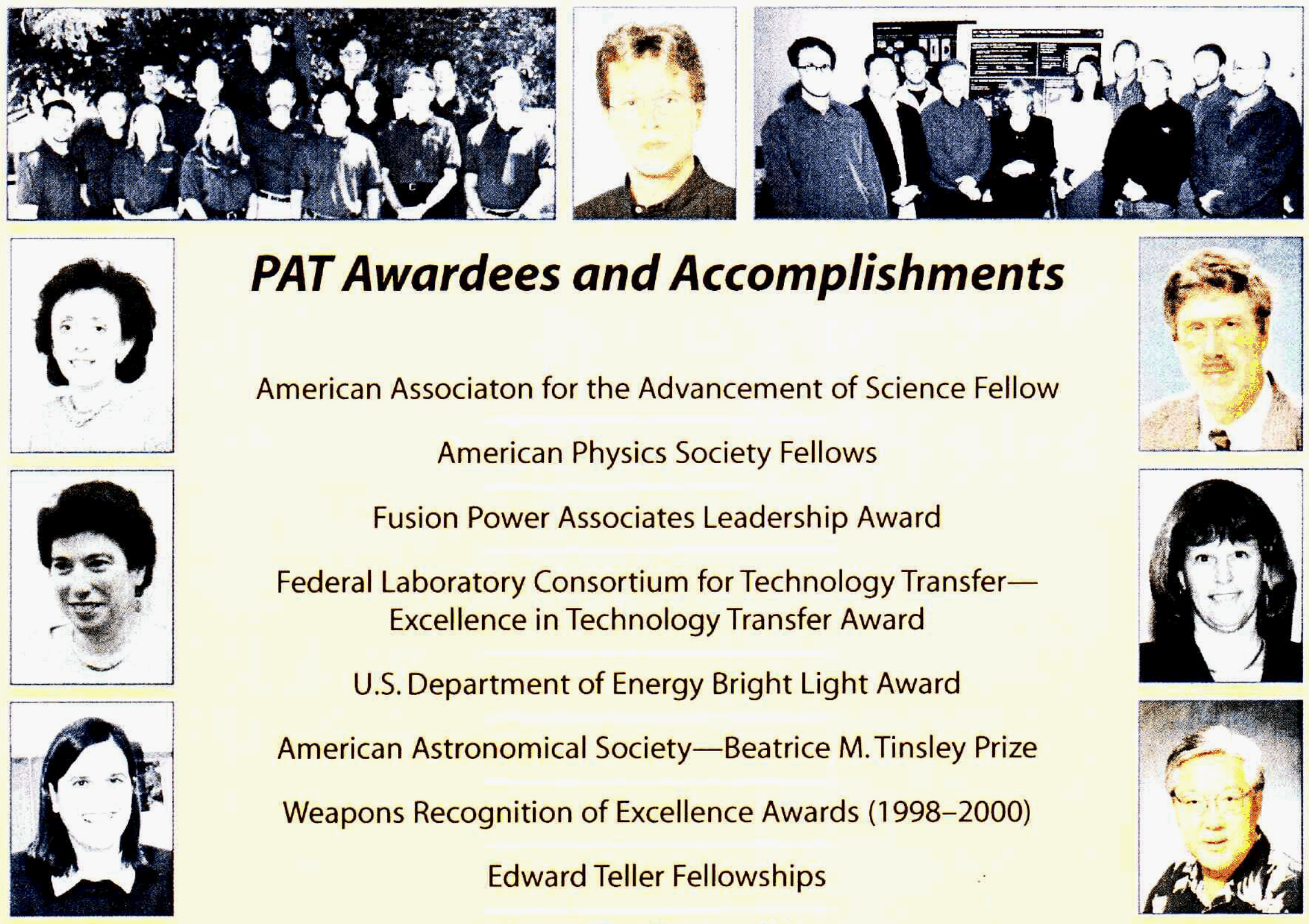

\section{PAT Awardees and Accomplishments}

American Associaton for the Advancement of Science Fellow American Physics Society Fellows

Fusion Power Associates Leadership Award

Federal Laboratory Consortium for Technology TransferExcellence in Technology Transfer Award

U.S. Department of Energy Bright Light Award

American Astronomical Society-Beatrice M. Tinsley Prize Weapons Recognition of Excellence Awards (1998-2000)

Edward Teller Fellowships

Lybrand Certificate of Merit
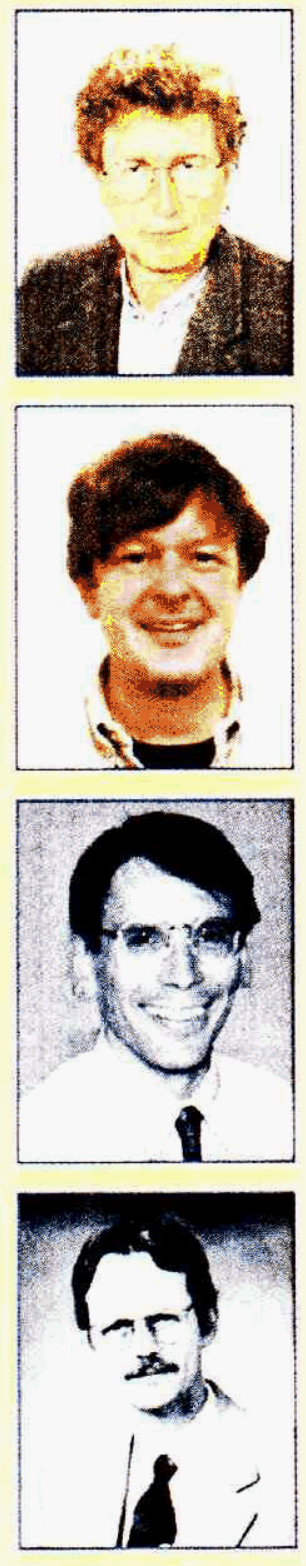

Use of Superconducting Tunnel Junction Detectors Featured in Synchrotron Radiation News

Unveiling of 10-Kilowatt Laser

Three-Year Extension of EUVL

PAT Awarded Two of LLNL's Eight University of California Campus-Laboratory Collaborative Projects and Exchanges

"Smart Probe" Development and Testing

Massive Compact Halo Objects Collaboration Findings

Guide Star Installed at Keck Observatory

New Defense Advanced Research Projects Agency Initiative

$$
\text { Publication of First Results from B Factory }
$$
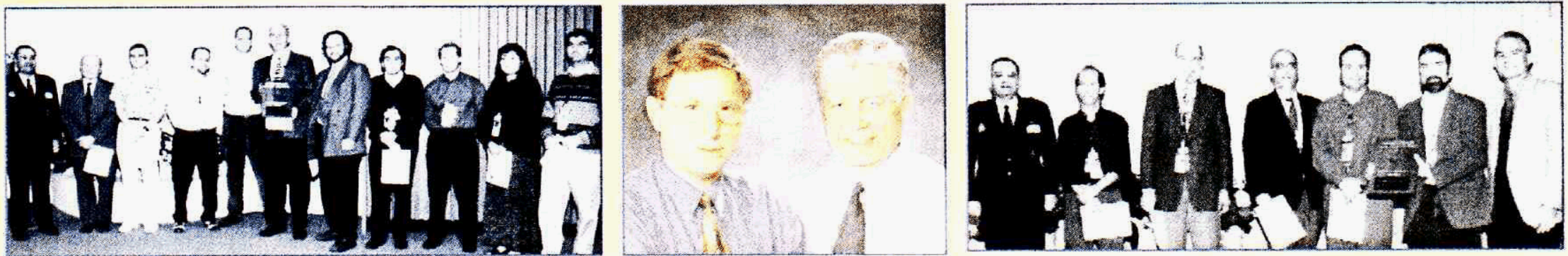


\section{Introduction}

\author{
By William H. Goldstein
}

The Physics and Advanced Technologies (PAT) Directorate was created in July 2000 by Bruce Tarter, Director of Lawrence Livermore National Laboratory (LLNL). The Director called for the new organization to execute and support programs that apply cutting-edge physics and advanced technology to develop integrated solutions to problems in national security, fusion energy, information science, health care, and other national grand challenges.

When I was appointed a year later as the PAT Directorate's first Associate Director, I initiated a strategic planning project to develop a vision, mission, and long-term goals for the Directorate. We adopted the goal of becoming a leader in frontier physics and technology for twenty-first-century national security missions: Stockpile Stewardship, homeland security, energy independence, and the exploration of space. Our mission is to:

- Help ensure the scientific excellence and vitality of the major LLNL programs through its leadership role in performing basic and applied multidisciplinary research and development with programmatic impact, and by recruiting and retaining science and technology leaders

- Create future opportunities and directions for LLNL and its major programs by growing new program areas and cuttingedge capabilities that are synergistic with, and supportive of, its national security mission
- Provide a direct conduit to the academic and high-tech industrial sectors for LLNL and its national security programs, through which the Laboratory gains access to frontier science and technology, and can impact the science and technology communities

- Leverage unique Laboratory capabilities, to advance the state of knowledge about our universe.

This inaugural PAT Annual Report begins a series that will chronicle our progress towards fulfilling this mission. I believe the report demonstrates that the PAT Directorate has a strong base of capabilities and accomplishments on which to build in meeting its goals. Some of the highlights include:

- Leadership of the Laboratory's Physical Data Research Program that provides fundamental physics information for the Stockpile Stewardship Program.

- Development of the handheld Microbead Immunoassay Dipstick System that will allow relatively untrained firstresponders to run sophisticated onsite diagnostics for pathogens, including those associated with biowarfare agents, by using a simple, onestep measurement.

- Major advances in target design for inertial fusion energy research using both laser and ion-beam drivers.

- Development of the Advanced Technology Kill Vehicle concept for use as a high-performance interceptor in a broad range of missile defense programs.
Over the course of the past decade, the Laboratory has seen its major program evolve from weapons research, development, and testing, to Stockpile Stewardship. Today, the country's national security priorities are changing rapidly: nuclear security is becoming a broader set of missions, and the Laboratory is being asked to contribute to a range of new mission areas from countering bioterrorism to ensuring information security. As we embark on the twenty-first century, the new PAT Directorate is poised to help lead the Laboratory's response to the country's changing national security needs.

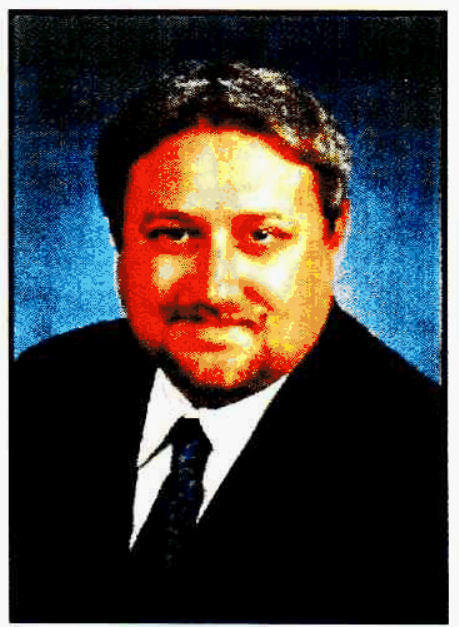

William H. Goldstein is Associate Director, Physics and Advanced Technologies

Directorate 


\section{Highlights from Physics and Advanc}

\section{High-Energy-Density Physics and Astrophysics (V Division)}

- V Division researchers conducted a series of scaled experiments at the Omega Laser facility (located at the University of Rochester) to investigate laser absorption and conversion to $\mathrm{x}$ rays, plasma fillings and hole closures, and laser-plasma instabilities in Inertial Confinement Fusion (ICF) targets. These experiments will test physics models in V Division design codes and improve target designs for National Ignition Facility (NIF) experiments in support of the Stockpile Stewardship Program.

- Detailed Term Accounting computation methodology was developed to analyze transport and spectral emission characteristics of mid- $Z$ plasmas at astrophysically redundant low densities and temperatures. This approach will be compared to statistical predictions to analyze high-energy-density experiments on NIF and Sandia's Z machine.

- The Djehuty Project developed a threedimensional (3-D) stellar evolution code operating on massively parallel computing machines using the best available physical data (e.g., opacities and equations of state [EOS]) as well as new algorithms. The project successfully executed the first ever 3-D simulation of a whole star, including convection processes.

\section{Condensed Matter Physics (H Division)}

- For the Stockpile Stewardship Program, H Division expanded and improved its library of EOS tables used by the Advanced Simulation and Computing (ASCI) simulation codes.

- In collaboration with LLNL's Center for Applied Scientific Computing, $H$ Division researchers produced the first Quantum Molecular Dynamics
(QMD) simulation of a shock wave in liquid deuterium. QMD is a method in which the motion of individual atoms is traced using forces determined by electronic structure methods. Analysis yields estimates for the pressure and the density in the medium.

- Positron annihilation spectroscopy was used as a nondestructive evaluation technique to determine the effects of aging on plutonium in the stockpile.

\section{Nuclear, Particle, and Accelerator Physics (N Division)}

- N Division achieved a major goal for the Physical Data Research Program in support of the Stockpile Stewardship Program-a precise (within 10\%) measurement of the ${ }^{239} \mathrm{Pu}(\mathrm{n}, 2 \mathrm{n}){ }^{238} \mathrm{Pu}$ cross section. The cross section is used in one major diagnostic in the interpretation of underground nuclear tests.

- Very-high-energy protons, used as a radiographic probe, were investigated as a technique for the Advanced Hydrodynamics Facility, which could be a major facility for the Stockpile Stewardship Program. Also, quantitative measurements of the dynamic behavior of materials under shock were conducted using high-energy protons as a radiographic probe.

- Under the direction of $\mathrm{N}$ Division physicists, LLNL is a member of a fourlaboratory consortium (Stanford Linear Accelerator Center [SLAC], Fermi National Accelerator Laboratory [FNAL], Lawrence Berkeley National Laboratory [LBNL], and LLNL) in the design of a teraelectronvolt-scale electron-positron linear collider. This behemoth facility would consist of two opposing linacs, be 20 miles long, and cost around $\$ 5$ billion. The prime LLNL contribution is the development of a photon collider experiment within this project. 


\section{ed Technologies Directorate 2001}

Fusion Energy Program (FEP)

- FEP maintained a leading role in the ongoing collaborative research program on the DIII-D National Fusion Facility in San Diego, California. This effort is an integral part of the overall program of improvement of the tokamak concept, which has been used to advance the science of high-temperature plasmas in magnetic fusion research.

- The Sustained Spheromak Physics Experiment (SSPX) developed new and enabling diagnostics. The spheromak, an advanced and alternate plasmaconfinement concept, has an internal dynamo to create its confining magnetic field.

- The Heavy-Ion-Fusion Virtual National Laboratory (HIF-VNL) is a coordinated Inertial Fusion Energy (IFE) research program among Princeton Plasma Physics Laboratory, LBNL, and LLNL. It is intended to maximize IFE progress. The success of the coordinated program is evidenced by three new major experiments.

- The IFE Target Design Program made major advances in designing new targets and variations of older designs using both laser drivers (such as the NIF) and heavy-ion beams.

\section{Institute of Geophysics and Planetary Physics (IGPP)}

- IGPP researchers installed a prototype adaptive optics system, used to correct atmospheric aberrations in incoming light, at the University of California's Lick Observatory on Mount Hamilton near San Jose. Subsequently, LLNL researchers helped to develop and install an even larger adaptive optics system at the Keck Observatory atop
Mauna Kea in Hawaii (see front cover photo). Data quality surpasses that from the space-borne Hubble Telescope.

- IGPP scientists used the Keck adaptive optics system to investigate one of the fundamental mysteries of astronomy: the nature and origin of supermassive black holes in the centers of galaxies. Highresolution images show that galaxies and black holes may grow in time through the merging of smaller systems.

- IGPP scientists continued to study the Milky Way's structure and composition by using the database from the Massive Compact Halo Objects (MACHO)

Project. Key results were the direct detection and characterization of a lens toward the Large Magellanic Cloud (LMC), as reported in the journal Nature; the first direct detection of a gravitational microlens; and the use of Hubble Space Telescope data to support a halo location for most of the lenses seen toward the LMC.

\section{Medical Technology Program (MTP)}

- A handheld device, the Microbead Immunoassay Dipstick System (MIDS), will allow relatively untrained personnel, such as firefighters and paramedics, to run sophisticated, onsite diagnostics for pathogens using a simple, one-step measurement. This point-of-care medical diagnostic is also intended for use at a patient's bedside; in third-world countries, military operations, or space travel; and as field diagnostics for environmental sampling and detection of biowarfare agents.

- MTP's Glucose Sensor Project is developing an artificial biomechanical pancreas by combining existing glucose insulin pumps with a continuous glucose sensor that is under development. The glucose sensor will 


\section{Highlights from PAT Direct}

continuously monitor blood-sugar levels and communicate to the insulin pump when to release insulin into the bloodstream, allowing diabetics to maintain correct insulin/blood-sugar levels minute-to-minute throughout the day and night (see back cover photo).

- MTP researchers collaborated with BioLuminate, Inc., to develop, produce, and market a revolutionary diagnostic device for early breast-cancer diagnosis. The biopsy probe uses four distinct physical detection methods within each "smart needle" to improve diagnosis: optical scattering and absorption spectroscopy, optical coherence domain reflectometry, near-infrared fluorescence, and a spectroscopic impedance measurement capability.

\section{Extreme Ultraviolet Lithography (EUVL) Program}

- Under the sponsorship of an integratedcircuit manufacturing consortium led by Intel, LLNL, LBNL, and Sandia National Laboratories/California (SNL/California) built an engineering test stand that can print a 25 -millimetersquare image with 70-nanometer resolution and uses a soft $x$-ray wavelength of 13.4 nanometers.

- On April 11, 2001, more than 200 people attended the EUVL Milestone Celebration to recognize the first operation of the EUVL tool built by the three national laboratories. Dignitaries included (see front cover photo) Dr. Craig Barrett, Chief Executive Officer, Intel Corporation; General John Gordon, Undersecretary for Nuclear Energy; Dr. Miriam John, Vice President,
SNL/California; the Honorable Ellen Tauscher, U.S. House of Representatives (Democrat); and Sunlin Chou, Senior Vice President and General Manager for the Technology and Manufacturing Group, Intel Corporation.

- In October 2001, EUVL, LLNL's largestever Cooperative Research and Development Agreement (CRADA), was extended for another three years. The CRADA is centered in the PAT Directorate.

\section{Imaging and Advanced Detectors Division (I Division)}

- A collaborative team led by the PAT Directorate was selected for funding support (approximately $\$ 9.5$ million) as the Coherent Communications, Imaging and Targeting Program through the U.S. Department of Defense's Defense Advanced Research Projects Agency (DARPA). The team is developing powerful, new capabilities for secure, free-space communication links (at rates of multigigabits per second) and aberration-free, 3-D imaging and targeting at very long ranges (more than 1,000 kilometers) featuring innovative optics concepts and integration of micro-electromechanical-systems (MEMS) spatiallight modulators.

- Support from the National Science Foundation's Center for Adaptive Optics will allow I Division researchers and collaborators to test the use of MEMS deformable mirrors to correct aberrations of the eye. Over a two-year period, the team will develop and test clinical ophthalmic instruments using 


\section{orate 2001 continued}

MEMS adaptive optics in hopes of revolutionizing the diagnosis and treatment of diseases that cause blindness and the techniques for vision correction in the general population.

- Significant progress was achieved in the development of high-resolution and high-sensitivity detectors for use from the near-infrared spectral region to gamma-ray wavelengths. At gamma-ray energies, the detectors can measure the ratio of nuclear isotopes, providing high precision for nonproliferation and arms control applications.

\section{Advanced Interceptor Technology Program (AITP)}

- AITP successfully developed a concept - the Advanced Technology Kill Vehicle (ATKV) - for a highperformance interceptor for use in a broad range of missile defense programs.

\section{Laser Science and Technology (LS\&T) Program}

- With support of the U.S. Army's Space and Missile Defense Command and in collaboration with industrial partners, the LS\&T Program is developing a highaverage-power (100-kilowatt-class), diode-pumped, solid-state, heat-capacity laser technology for application in tactical, short-range air defense missions.
- LS\&T Program developed new frontend technology utilizing optical-parametric, chirped-pulse amplification (OPCPA) to replace the regenerative amplifier used in a variety of chirpedpulse amplifiers. Wavelength and pulse-energy levels obtained from this OPCPA are ideal for seeding kilojouleclass Nd:glass amplifiers, such as the NIF, Falcon Laser, and other highenergy, ultrashort-pulse laser systems.

\section{Institute for Laser Science and Applications (ILSA)}

- ILSA initiated a study of self-induced plasma smoothing to describe various temporal and spatial nonlinear processes that affect the coupling of intense laser light to targets such as those used in the Stockpile Stewardship Program and inertial confinement fusion.

- ILSA researchers used laser-produced plasma as a lens to focus gigaelectronvolt electrons and positrons at SLAC. The plasma lens has been proposed as a final focusing mechanism to achieve high luminosity for future high-energy particle colliders.

- ILSA researchers used Compton scattering as a source of tunable $x$-rays for application to protein crystallography. Together with recombinant DNA technology, x-ray protein crystallography establishes a new paradigm for drug design and synthesis. 


\section{High-Energy-Density Physics and Ast}

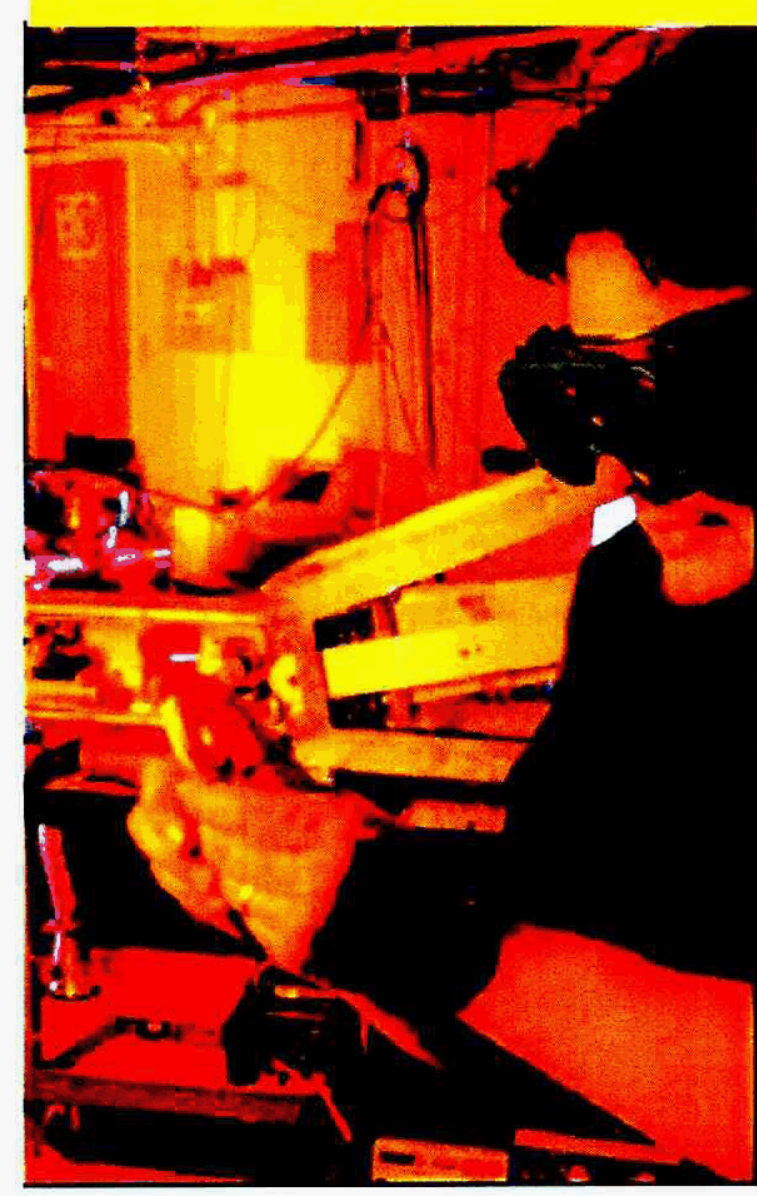

A PAT technician inspects an LLNLdesigned spectrometer undergoing calibration with a laser-plasma x-ray monochrometer.
The mission of the PAT Directorate's High-Energy-Density Physics and Astrophysics Division (known as $\mathrm{V}$ Division) is to advance the understanding of the physical properties of matter at the extreme conditions of temperature and density (up to stellar temperatures and pressures) where materials are significantly ionized. These physical properties include radiative opacity, equation of state (EOS), and atomic kinetics and spectroscopy of nonlocal thermodynamic equilibrium plasmas. These physical properties determine the creation, evolution, and energy balance of high-temperature plasmas (gases containing electrically charged particles).

\section{$\mathrm{V}$ Division's expertise in high-energy-} density physics supports its development of computer modeling of atomic processes that are crucial to major programs at LLNL, principally the Stockpile Stewardship Program and the National Ignition Facility (NIF) Programs Directorate. To develop quantitative models and databases, $V$ Division combines state-of-the-art theories with advanced computations. These models are tested through laboratory experiments on plasmas at known thermodynamic conditions and, in some cases, tested against observations of astronomical phenomena.

The high-energy-density research program of $\mathrm{V}$ Division shares areas of common interest with astrophysics. These common areas include the physical properties of opacities, spectroscopy, the EOSs of plasmas, the development and utilization of radiation hydrodynamic simulation codes, and imaging and spectroscopy instrumentation. Instrumentation also strongly overlaps with other LLNL programs in nonproliferation and intelligence. Because of these synergies,

$\mathrm{V}$ Division undertakes a broad-based collection of observational and computational astrophysics projects. These projects allow V Division to apply its expertise to other national programs, benefit from developments of a large international community that shares many common interests, and recruit talented young scientists who have expertise in areas important to Laboratory programs.

During the past year, $\mathrm{V}$ Division research activities have produced a number of important advances that are highlighted in the following sections.
Fig. 1. The new parallel high- $Z$ opacity code has been tested by comparisons with iron (Fe) opacity measurements at low density, including approximately 50 times more transitions than could be computed in a similar time on a serial computer. Understanding and computing the crossover between detailed term accounting and statistical opacity methods will contribute to the analysis of high-energy-density experiments.

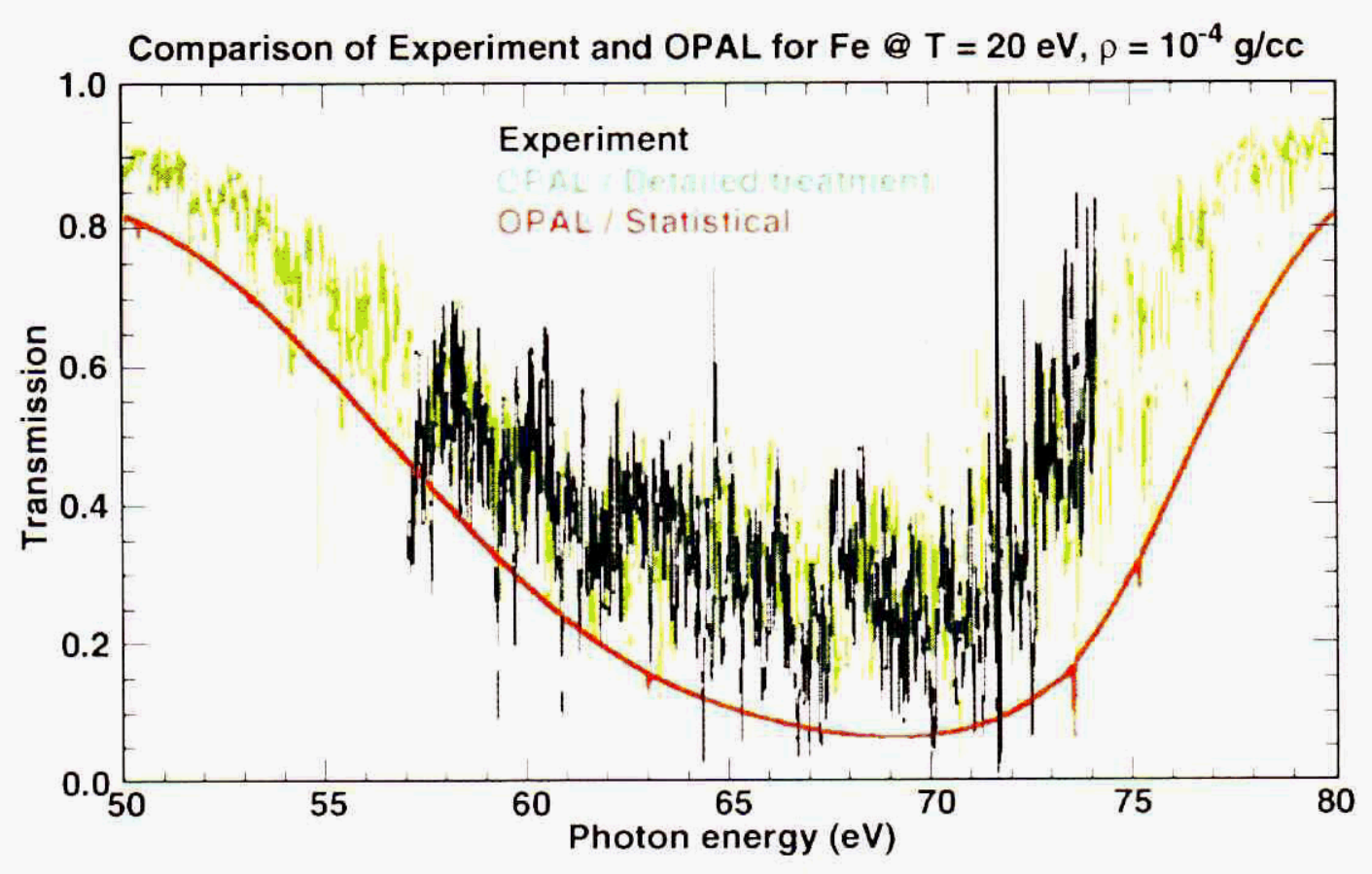




\section{rophysics Division (V Division)}

First-generation Detailed Term Accounting Opacity Code for Heavy Elements

The analysis of transport and spectral emission characteristics of plasmas with large ion-bound state populations is complicated by the presence of numerous closely spaced transitions. Two approaches have been applied to this situation. Detailed term accounting (DTA) attempts to explicitly compute the contributions of every significant transition. On the other hand, statistical approaches, such as the super transition array (STA) method, use theorems about the features of large transition arrays to assemble an approximate opacity model.

Computations and experiments for mid- $Z$ elements at astrophysically relevant low densities and temperatures have revealed that, for these conditions, the DTA approach is required because of the significant porosity between the distinct transitions. For example, Fig. 1 shows a comparison of Saturn pulsed power experimental and OPAL theoretical opacities for iron, demonstrating the need for a DTA treatment at mid-Z. Researchers are focusing attention on computing and understanding the comparison between detail and statistical predictions to analyze high-energy-density physics experiments to be done at the NIF and the Sandia National Laboratories (SNL) Z machine.

Based on previous successes with mid-Z DTA computations, V Division personnel attacked the problem of scaling DTA to higher Z. As $Z$ increases, the number of relevant quantum mechanical states and transitions explodes, necessitating scalable parallel computing. While the initial, working version of the code TOPAZ uses a simple form of parallelism, the ultimate scaling of this code to higher densities and temperatures will rely on significant innovation in the treatment of atomic structure.

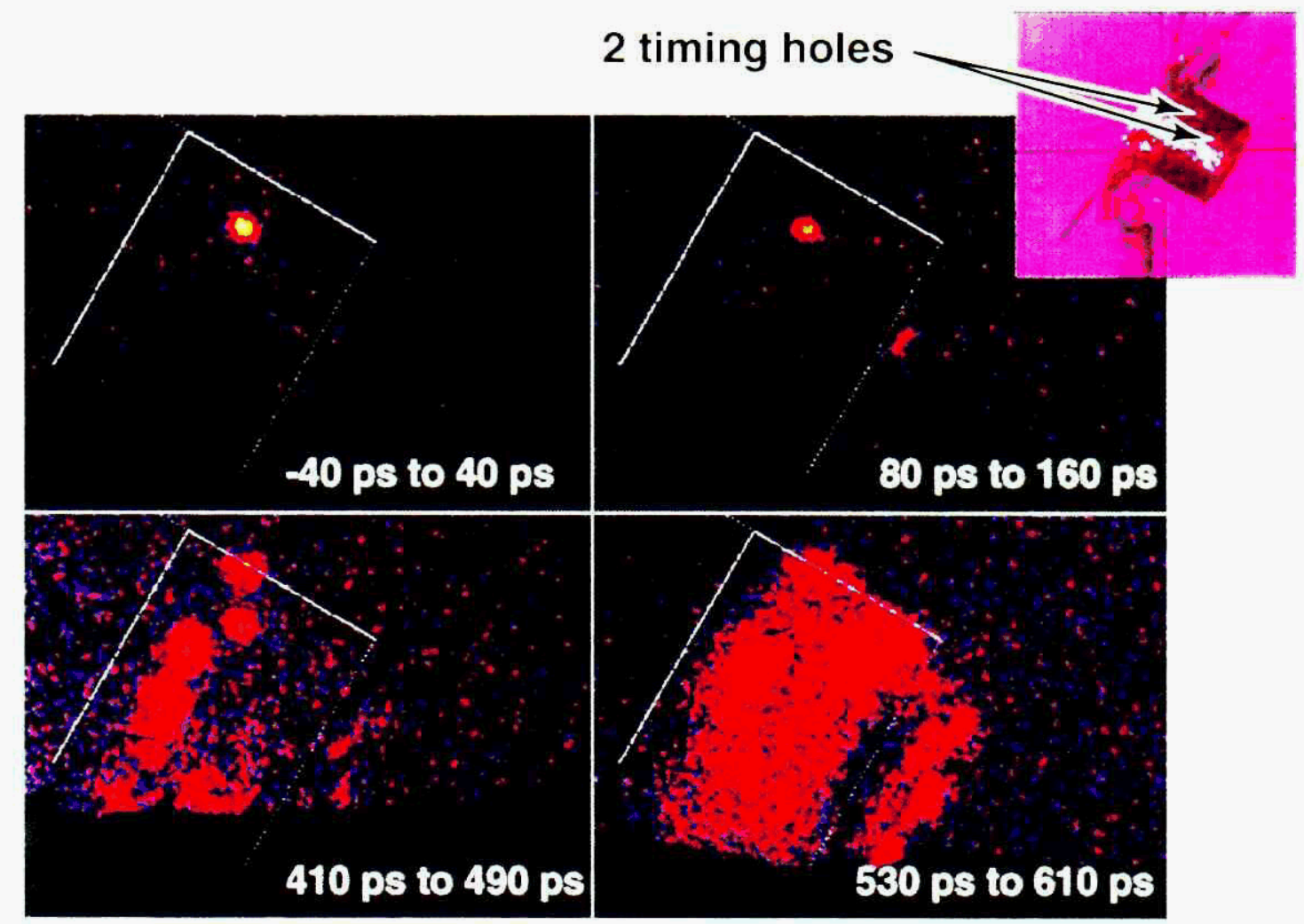

Development of High-temperature Holhraums and Diagnostic Techniques

The NIF will provide significant new capabilities for $\mathrm{V}$ Division research programs and will enable the performance of experiments at previously inaccessible hightemperature conditions. However, producing and utilizing these extremely high-energy-density regimes will require new experimental techniques and different target designs than previous experiments on the Nova Laser at LLNL and the Omega Laser at the University of Rochester in New York state. In particular, the planned opacity experiments require a better understanding of the performance of hightemperature hohlraums (fuel capsules).

These hohlraums are significantly outside the design parameters of the ignition hohlraums developed by the inertial confinement fusion (ICF) community. In order to understand the performance of these hotter hohlraums, V Division researchers are conducting a series of scaled experiments at the Omega Laser facility to investigate laser absorption and conversion to $x$-rays, plasma fillings and hole closures, and laser-plasma instabilities in these small hohlraums. As shown in Fig. 2, these experiments will be used
Fig. 2. Snapshots of small-scale hohlraum experiments measuring the radiation wave burning through the hohlraum wall provide data that can be used to infer the hohlraum temperature. 
Fig. 3. In the Isentropic Compression Experiments (ICE), a ramped current is used to generate a gently rising magnetic pressure wave to compress a sample without generating an abrupt shock wave. The Velocity Interferometry System for Any Reflector (VISAR) diagnostic measures the velocity of the sample interface as a function of time. The velocity profiles from two samples of varying thicknesses can be used to determine the equation of state (EOS) of the material. to test physics models in $\mathrm{V}$ Division design codes and to improve the target designs for the NIF experiments.

\section{Isentropic Compression Experiments Using Magnetic Field Drivers}

To date, most high-pressure measurements of EOS have been performed along the principal Hugoniot (that is, the set of states reached by single shocks of varying pressure). Critical steps are extending measurements to higher pressures and obtaining data away from the principal Hugoniot, especially because the largest differences between theoretical models occur at conditions where few data exist to guide the theories. In recent years, V Division personnel have collaborated with SNL to develop an application of the Z-pulse power facility to Isentropic Compression Experiments (ICE).

In these experiments, a large, ramped magnetic field shocklessly compresses samples to pressures in excess of 400 kilobar. Measurements from the Velocity Interferometry System for Any Reflector (VISAR), which is a laser interferometry technique to measure moving interfaces, are used to obtain velocitywave profiles that can be analyzed to yield EOS and strength data (Fig. 3). During the past year, this technique was applied to study basic science and programmatic problems. V Division investigators studied the phase diagram of iron and measured the EOS and properties of materials requested by the Secondary Design Program.

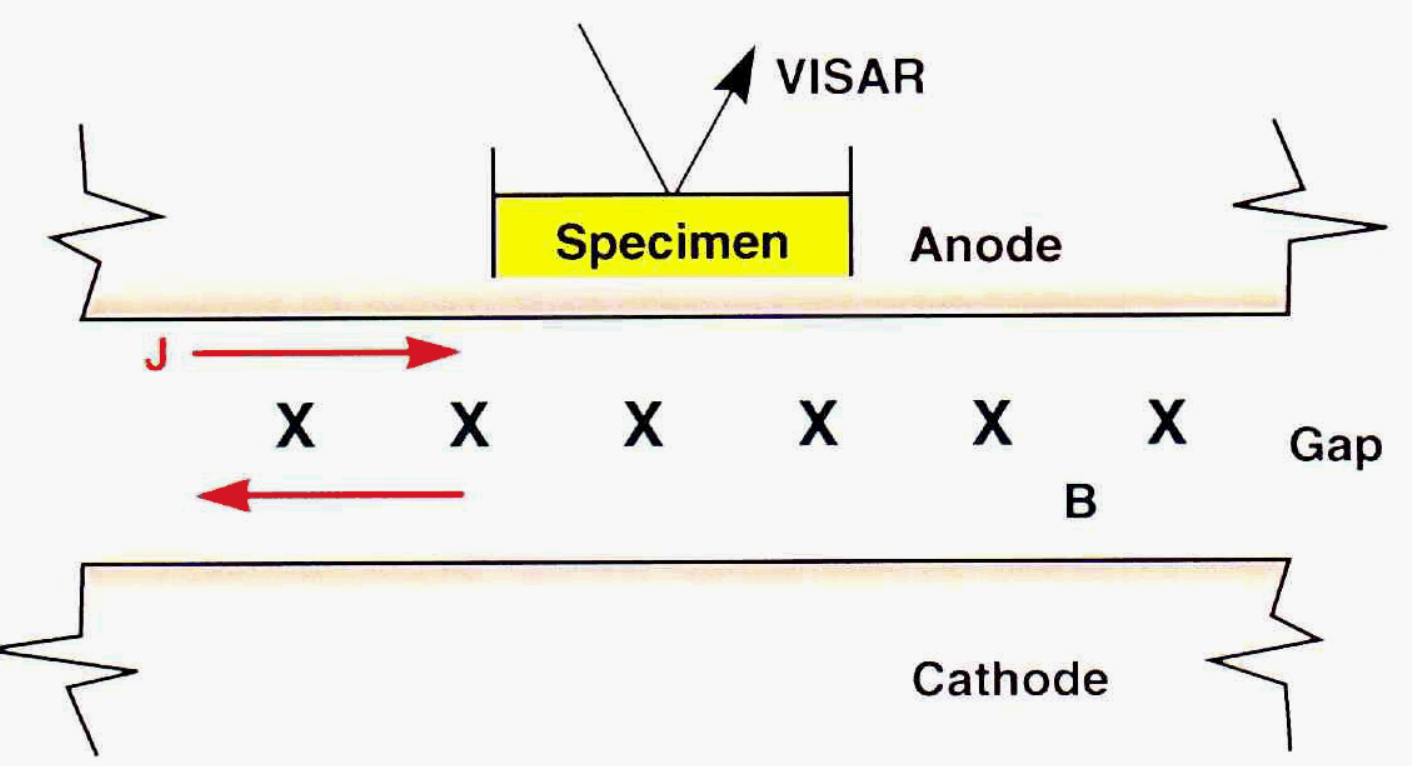

As part of a project conducted with Laboratory Directed Research and Development (LDRD) funds, a series of experiments studied these ultrahighintensity laser interactions. The results demonstrate that the latter production mechanism is the operative one. $V$ Division researchers are currently developing techniques to use these protons to volumetrically heat and radiograph samples of material to warm dense plasma conditions (Fig. 4). These techniques will be applied to the study of opacity and EOS. 


\section{$X$-ray Emissions from Comets}

$X$-ray satellite observations have firmly established comets as x-ray sources, a discovery that has far-reaching implications for understanding their interaction with the inner solar system. This discovery has opened up a new wavelength band for probing the solar windspace weather-in real time, studying interactions between comets and the sun, and understanding the composition of cometary atmospheres. However, because the charge transfer of solar-wind heavy ions, which have been proposed as the comet's main x-ray production mechanism, is poorly understood, models of charge-transfer-induced $x$-ray emission are based on best-guess estimates. Improving the understanding of these $x$-ray production mechanisms greatly benefits LLNL's core mission in high-temperature plasma and atomic physics and broadens LLNL's expertise in modeling short-wavelength radiation sources.

The goals of this project are to:

- Simulate, in a controlled laboratory setting, the conditions that cause comets to emit $x$-rays

- Produce the necessary experimental database for describing charge-transferinduced $x$-ray emissions in low-energy collisions of highly charged ions with atoms and molecules. One such result is shown in Fig. 5.

- Incorporate these results into models that accurately describe $x$-ray emissions by comets.

During the course of ongoing studies, several previously unexplored processes in the cometary $x$-ray models have been considered:

- The x-ray emission lines are a diagnostic of the solar-wind velocity because the relative magnitude of the various lines changes with the collision energy between the ion and the neutrals.
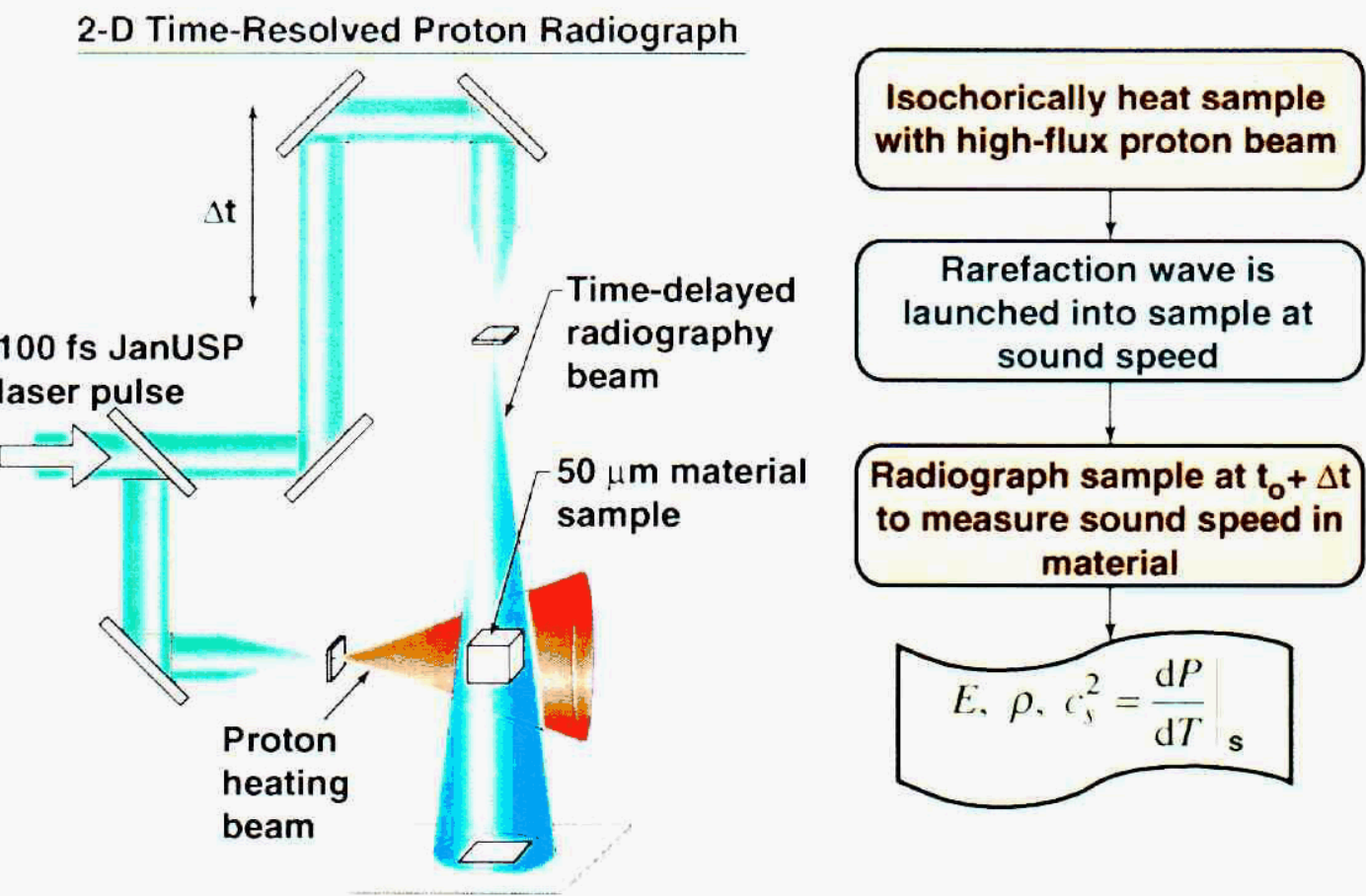

Fig. 4. The intense proton beam produced in high-intensity laser interactions is being used to heat and radiograph samples in order to study material properties of warm dense plasmas.

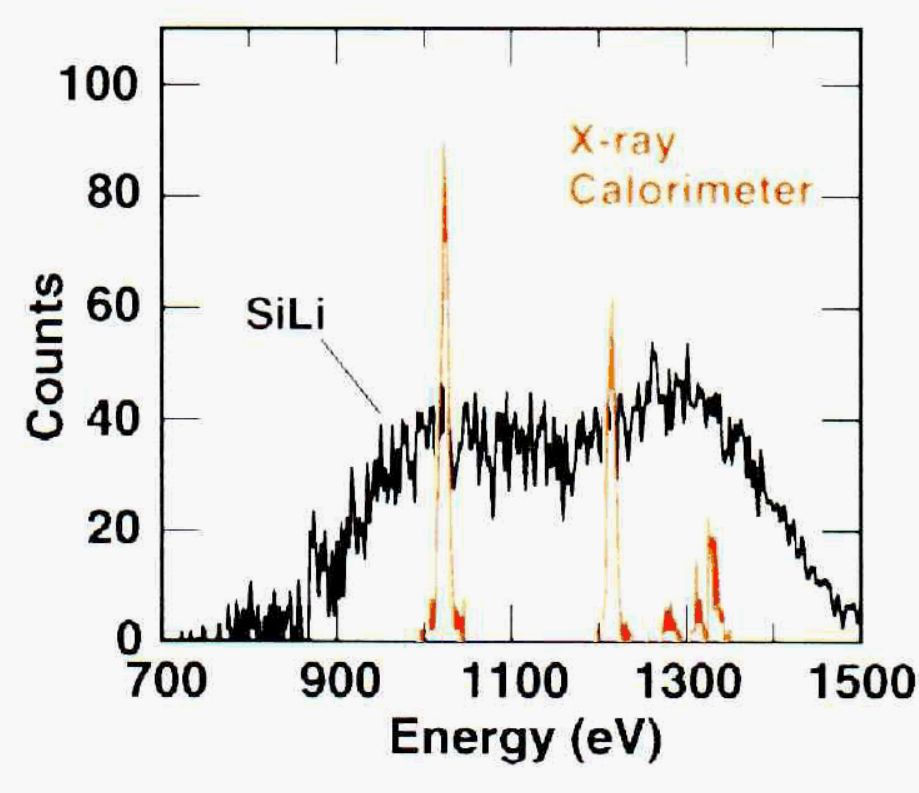

- Double- and multiple-electron capture can make up $50 \%$ of the capture processes. This capture rate changes the shape of the $x$-ray spectrum significantly, yet the process is completely absent in present cometary models.

- The detailed emission also depends on the chemical composition of the cometary gases. This knowledge may provide a diagnostic of the chemical composition of the comet head, thus increasing our understanding of primordial matter emitted by comets.
Fig. 5. The spectrum of charge-exchangeinduced K-shell $\mathrm{x}$-ray emission from $\mathrm{Ne}^{9+}$ measured by the highresolution

microcalorimeter (red curve) reveals a wealth of details for the emission process that cannot be discerned in measurements by a conventional solidstate $x$-ray detector (black curve). 
Fig. 6. A 3-D simulation using Djehuty shows the onset of convective flows in the core of a four solar mass star. A quantitative understanding of these flows is an outstanding problem in the theory of stellar evolution.

\section{Computational Modeling of Three-dimensional Hydrodynamic Processes in Stars}

Stars provide the fundamental quantitative units for measuring the universe. They are the foundation for determining distances and ages, and they are the factories driving the chemical evolution of the universe. Stars continue to be used as physics laboratories for constraining the properties of fundamental particles, such as cross sections and masses, as well as the properties of hot plasmas, including their opacity and EOS.

Current practices in stellar evolution employ one-dimensional calculations that quantitatively apply to only a minority of the observed stars (single, nonrotating stars, or well-detached binaries). Even in these systems, astrophysicists are dependent on models of complex, threedimensional (3-D) processes, such as convection. With the advent of massively parallel computers and the development of codes to use them, LLNI is uniquely situated to move the calculation of stellar properties to the next level of physical understanding. The Djehuty Project is a Strategic Initiative that has leveraged abilities from across the L aboratory, including the Defense and Nuclear Technologies and the PAT Directorates, the Center for Applied Scientific Computing, and Livermore Computing. The Djehuty Project

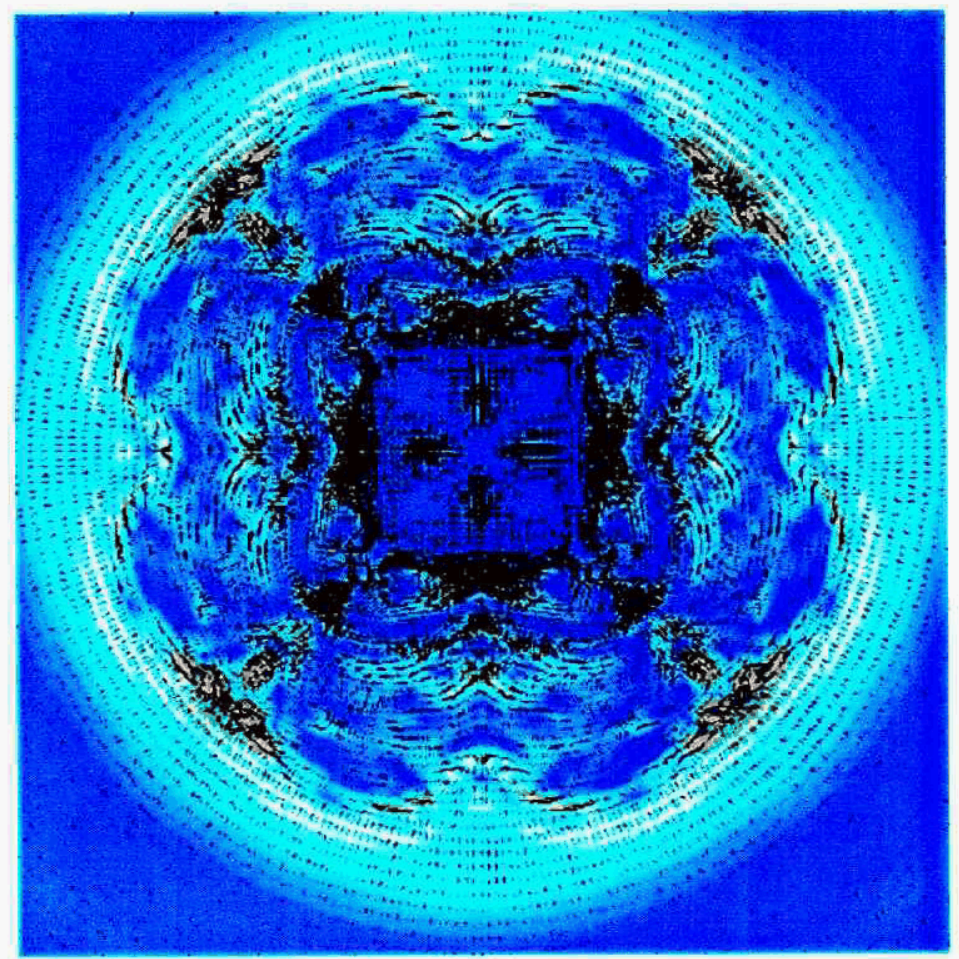

will develop a 3-D stellar evolution code operating on massively parallel machines (see front cover photo) with the best available physical data (e.g., opacities and EOS), as well as new algorithms (Fig. 6).

In September 2000, PAT researchers successfully executed a 3-D hydrodynamic simulation of a star on the TC $2 \mathrm{~K}$ computer system. This was the first ever $3-\mathrm{D}$ simulation of a whole star. The simulation was run on a single processor, using partial physics and an underresolved (400,000zone) mesh. This progress made possible a series of subsequent runs to study different mesh constructs, test and improve the accuracy of the 3-D models, and begin physics optimization.

The Djehuty Project now has an accurate EOS; astrophysical opacities for use with its radiative-diffusion transport package; and a nuclear-reaction network for hydrogen, helium, and carbon burning. The gravity implementation is currently complete for spherical stars only, but it is adequate for the first major 3-D science study of convective cores. Large (more than 50-million-zone) meshes needed for realistic stellar modeling have been constructed. Operations are moving from a parallel to a massively parallel environment, as the 3-D stellar models have now been decomposed and run in parallel operations, using up to 256 processors.

With the basic elements of Djehuty functional, LLNL investigators have made a number of extended parallel runs to address a long-standing, well-characterized discrepancy between one-dimensional stellar models and observed stars - an important validation step for presenting Djehuty to the astrophysical community. This testing has also permitted a number of code problems to be found and corrected. LLNL researchers have also begun to look at 3-D calculations of the Helium Flash, for which the time development of convection is critical, and of a nova explosion. 
Efforts have begun to document the code for publication to the astrophysical community, and preliminary results have been presented at a number of conferences. Algorithm development continues for both the physical processes and the data exploration necessary for studying rapidly rotating stars and binaries.

\section{Imaging Fourier Transform Spectrometer for Astrophysics}

The Imaging Fourier Transform

Spectrometer (IFTS) team completed the refurbishment of the IFTS instrument and fielded it five times this year-twice to the Lick Observatory near San Jose and three times to the Apache Point Observatory in the Sacramento Mountains of New Mexico. The Lick Observatory runs determined that the instrument functioned properly in the adverse environment of a mountaintop telescope. The Apache Point runs

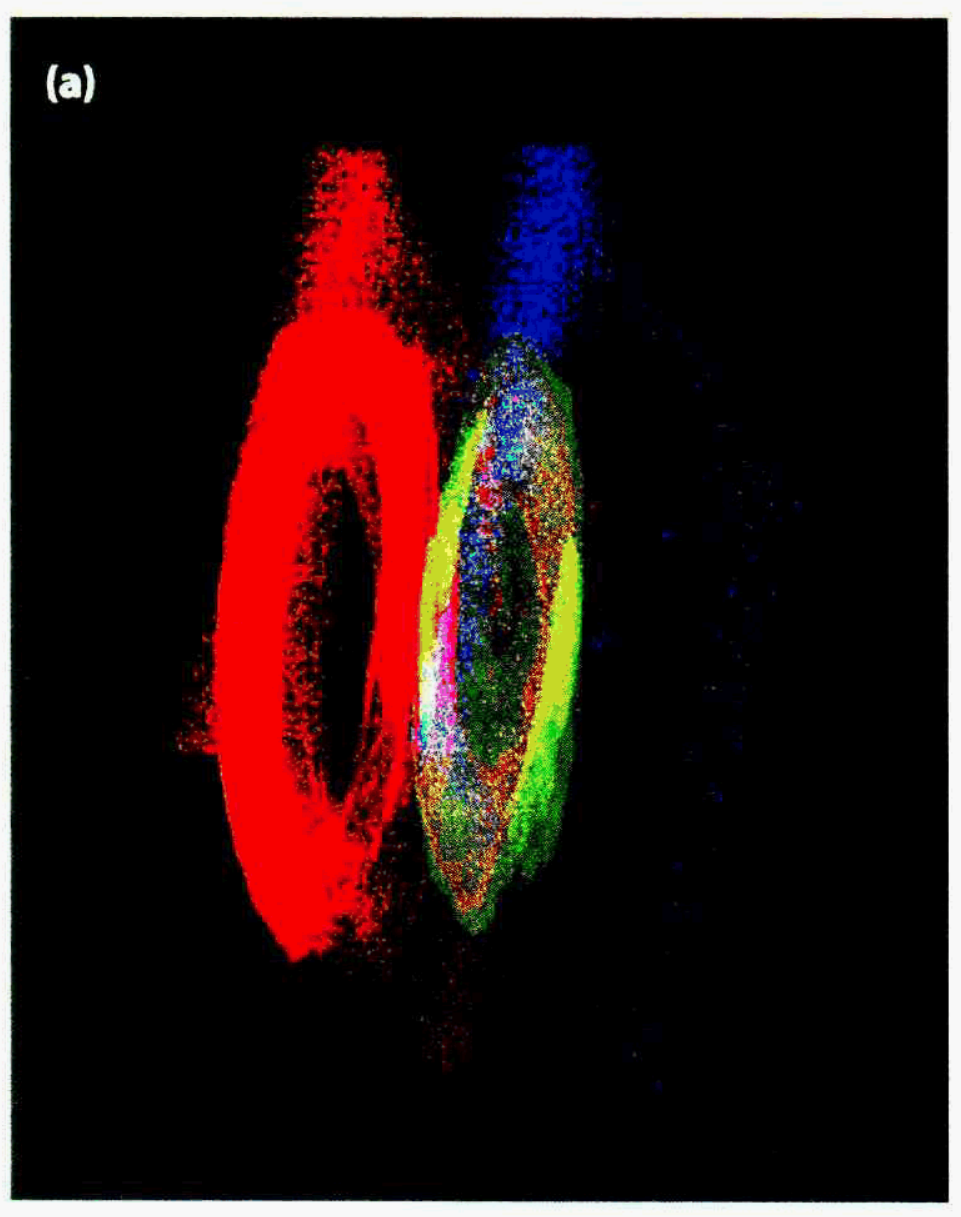

collected the first visible-band IFTS datacubes ever obtained. Fig. 7 shows a comparison of a distilled image to the same datacube in a $3-\mathrm{D}$ rendering.

L.LNL's IFTS instrument and its observations are being used to develop needed technologies and data-reduction techniques for step-scanned interferometry measured over long observation times. The IFTS instrument will also allow LLNL to pursue cutting-edge astrophysical observations, including imaging spectroscopythe best tool for investigating extended regions of varying chemical, thermal, and dynamical properties. The planned observations include studying the dynamics and formation of galaxies and galaxy clusters, thereby indirectly detecting their darkmatter components. V Division researchers are now proceeding to develop specialty tools for observation planning, data reduction, and data analysis.

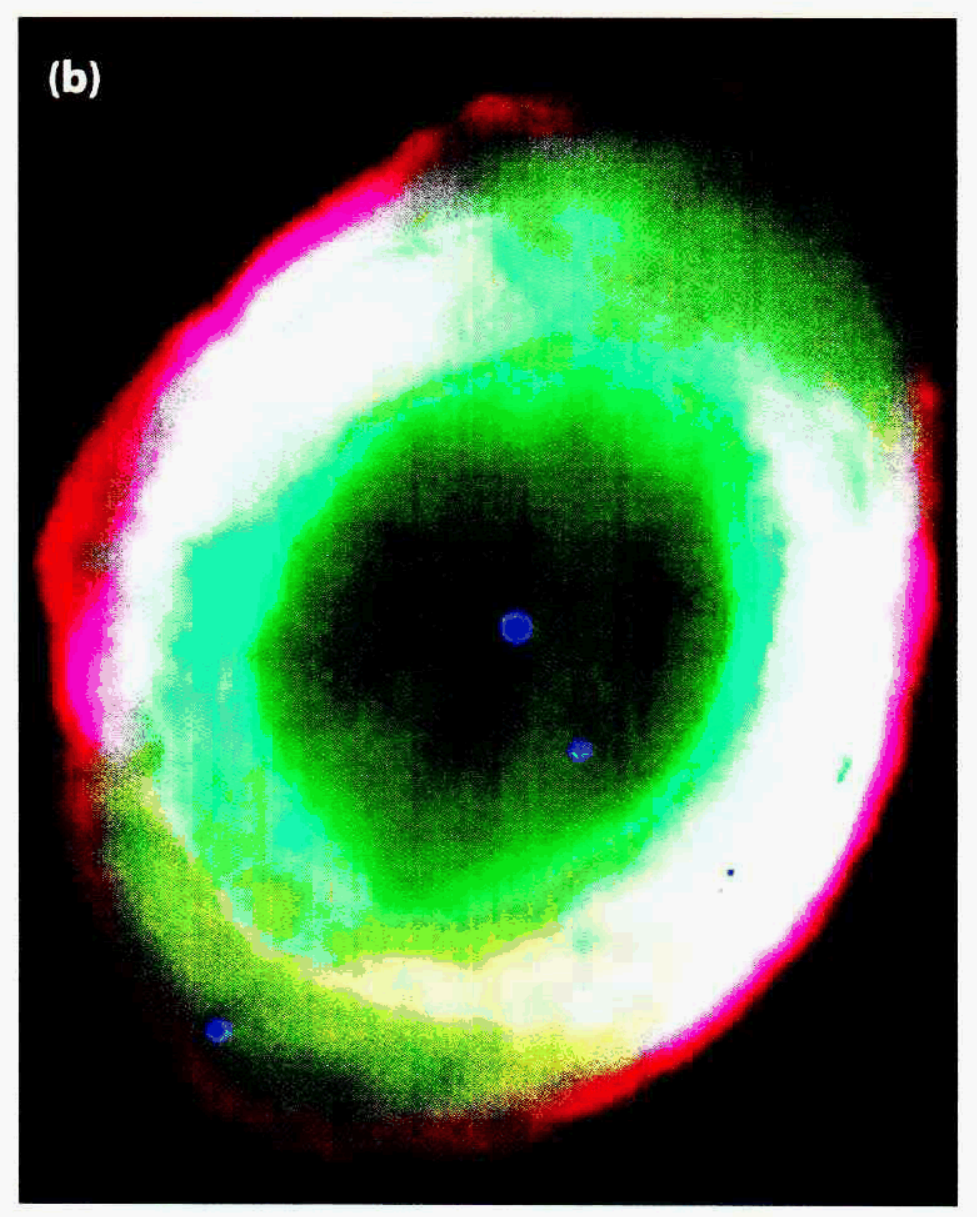

Fig. 7. Two views of an astronomical datacube obtained by LLNL's Imaging Fourier Transform Spectrometer (IFTS). (a) The first is a threecolor image of the Ring Nebula (M57) distilled from 220 color planes. (b) The second is a rendering of the same datacube in three dimensions. The vertical dimension is spectral frequency. The dominant red slice is $\mathrm{H}$-alpha at $6563 \AA$, and the dominant green slice is the Oxygen III doublet at $5007 \AA$ and $4959 \AA$. 


\section{Condensed Matter Physics Division}

The Condensed Matter Physics Division (known as H Division) combines unique experimental and theoretical capabilities to create a world-class center for research in condensed-matter physics and materials science. The Division emphasizes research on material properties and behavior at high pressures and other extreme or unusual environments. $\mathrm{H}$ Division supports and advances the fundamental science that underpins its mission, requiring that $\mathrm{H}$ Division personnel be world leaders in:

- High-pressure physics

- The development and application of advanced numerical methods for simulating materials properties and behavior

- EOS theory and materials data representation

- The development and application of state-of-the-art diagnostics and materials characterization tools.

H Division also has substantial responsibilities within the Stockpile Stewardship Program, providing experimental data and physics-based models of thermal and mechanical properties of materials to the weapons program in three key areas:

- EOS of materials, including melting and other phase transitions

- The constitutive properties of metals, especially plasticity, strength, ejecta, and spall

- EOS of high explosives (HE), HE detonation products, and other phenomena, such as the kinetics of energy release that affect HE performance.

For the Stockpile Stewardship Program, $\mathrm{H}$ Division maintains a library of EOS tables used by the Advanced Simulation and Computing (ASCI) simulation codes. ASCI is a program designed to computationally simulate the performance of an aging nuclear stockpile.
Because of its capabilities, $\mathrm{H}$ Division participates in many scientific fields beyond those defined by its program mission. For example, its expertise in high-pressure science contributes to geophysics and planetary science and to the creation of novel materials. Its unique simulation techniques have applications to computational biology and nanotechnology. The ability to characterize defects in metals with positron annihilation spectroscopy can be applied to advanced metallurgy.

H Division has produced many highquality research results in the past year. Some of the most important are highlighted below.

\section{First-principles Simulation of Shock Propagation}

$\mathrm{H}$ Division exploits ASCI to maintain expertise and develop new capabilities in a wide range of computationally intensive simulation techniques. One such method is Quantum Molecular Dynamics (QMD), in which the motion of individual atoms is traced using forces determined by electronic structure methods. Normally, such calculations are carried out at fixed temperature and density, and the statistically averaged results are used to estimate the EOS for a given material. This year, in collaboration with LLNL's Center for Applied Scientific Computing, H Division researchers produced the first QMD simulation of a shock wave in liquid deuterium. These simulations use the same basic QMD method except that one wall of the simulation cell is replaced by a rapidly moving impactor (shown in Fig. 8a). The simulation clearly shows the formation of a shock wave. Observation of the speed of the shock front then yields an estimate of the pressure and density on the Hugoniot (yellow boxes in Fig. 8b), which seems to be in reasonable agreement with the experiments performed on the Nova Laser. 


\section{Large-volume-collapse Transitions} in Rare-earth and Actinide Metals

Rare-earth and actinide metals undergo high-pressure phase transitions that exhibit very large volume collapses. It is believed that these transitions are driven by pressure-induced changes in the nature of the f-electrons from itinerant to localized. H Division personnel have made an extensive study of this phenomenon for the case of praseodymium.

The experiments utilized angle-resolved $x$-ray scattering in diamond anvil cells. The samples were prepared and loaded at LLNL. They were then taken to a synchrotron $x$-ray source to determine the volume and structure of the material from $x$-ray diffraction patterns. By heating the diamond anvil cells, researchers determined the size of the volume collapse as a function of temperature. These volumechange data, in conjunction with the slope of the phase-transition line, yielded an entropy change across the transition that is entirely consistent with theoretical expectations. Companion theoretical studies were also successfully tested this year for the analogous volume-collapse transition in cerium.

(a)

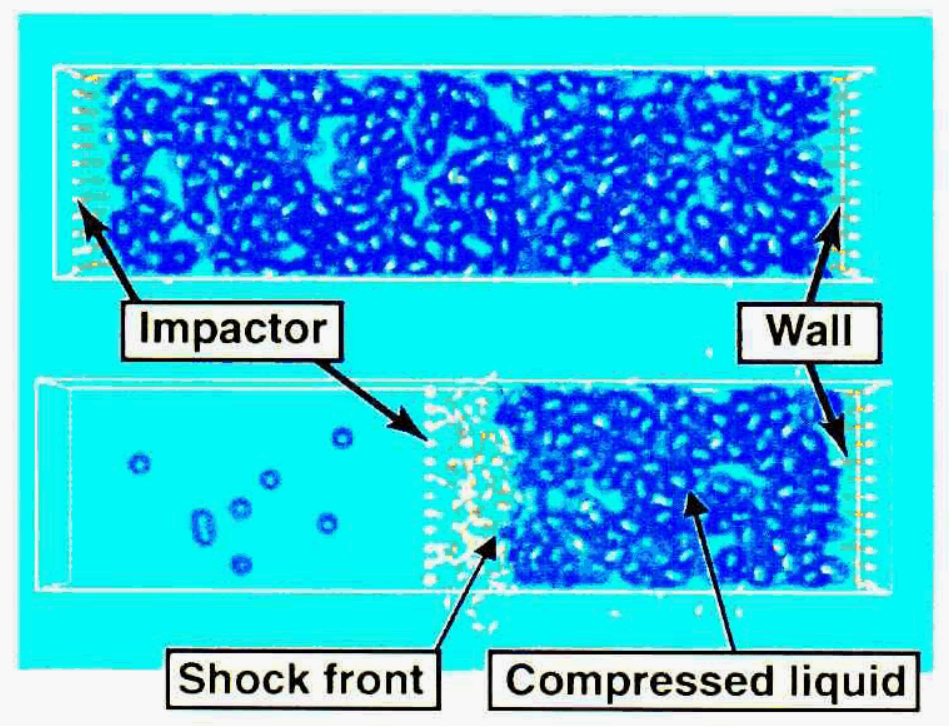

\section{Probing Defects in Aging Plutonium}

A critical task in the Stockpile Stewardship Program is to determine the behavior of materials in the stockpile, particularly the behavior of plutonium. One method used to perform nondestructive evaluation of the defects present in metals is positron annihilation spectroscopy. This technique relies on the facts that positrons are preferentially trapped within the open volumes created by defects in the metal and that their average lifetimes depend sensitively on the size of these defects. The fundamental experiment is to deposit positrons into a sample and measure the number of annihilation gamma rays produced as a function of time. Analysis of the data extracts various annihilation lifetimes that can be compared to those predicted by sophisticated electronic structure calculations for specific defect types.

\section{Joint Actinide Shock Physics Experimental Research Facility} Motivated by the need for accurate EOS information on the actinides, the U.S. Department of Energy (DOE) has invested heavily in the construction of the Joint Actinide Shock Physics Experimental Research (JASPER) Facility at the Nevada Test Site. The main feature of the facility is

(b)

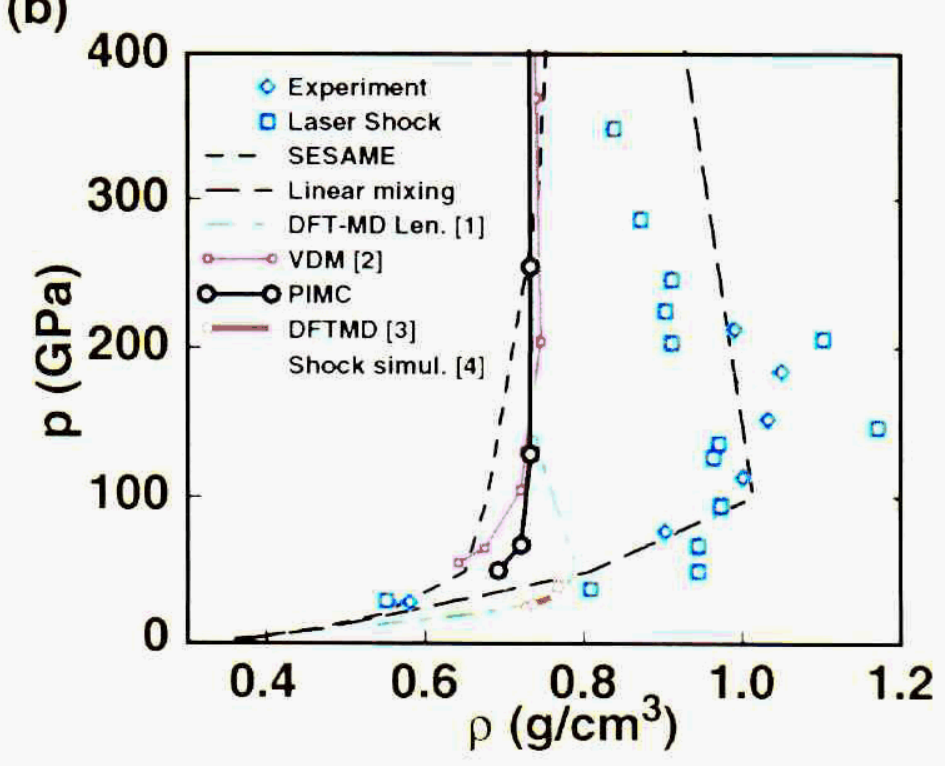

Fig. 8. (a) Quantum Molecular Dynamics (QMD) simulation of a shock wave in liquid deuterium. The impactor moves at a constant velocity from left to right, and the progress of the shock front is monitored as a function of time. (b) The simulated Hugoniot can be calculated from the known speed of the impactor and the "measured" speed of the shock front. The calculation can then be compared to the speed measured on the Nova Laser. 


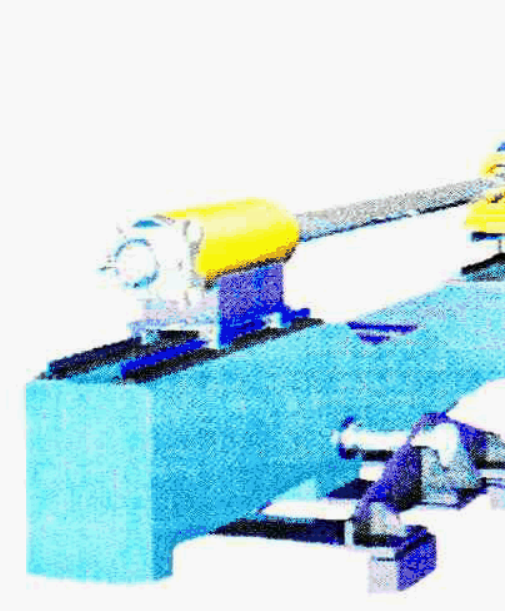

Fig. 9. The gas gun at the Joint Actinide Shock Physics Experimental Research (JASPER) facility at the Nevada Test Site will allow equation of state (EOS) and other measurements to be made on plutonium. a two-stage gas gun housed in a containment facility (Fig. 9). H Division has played a major role in the multilaboratory collaboration to bring the gas gun on line. During the past year, construction of the gas gun was completed; and several test shots were successful. The remaining work will complete the commissioning of the facility for "hot" operations.

The gas gun will permit hypervelocity projectiles to be fired into highly diagnosed targets, shocking matter to extreme conditions for a millionth of a second or less. These experiments will create pressures of a million-plus atmospheres and temperatures up to thousands of degrees, depending upon the material being shocked.

\section{Atomic-scale Simulation and Characterization of Dislocations}

The development of predictive models of strength and failure in metals requires an understanding of phenomena occurring on multiple-length scales, ranging from the atomic scale to the macroscopic continuum world. H Division's efforts to
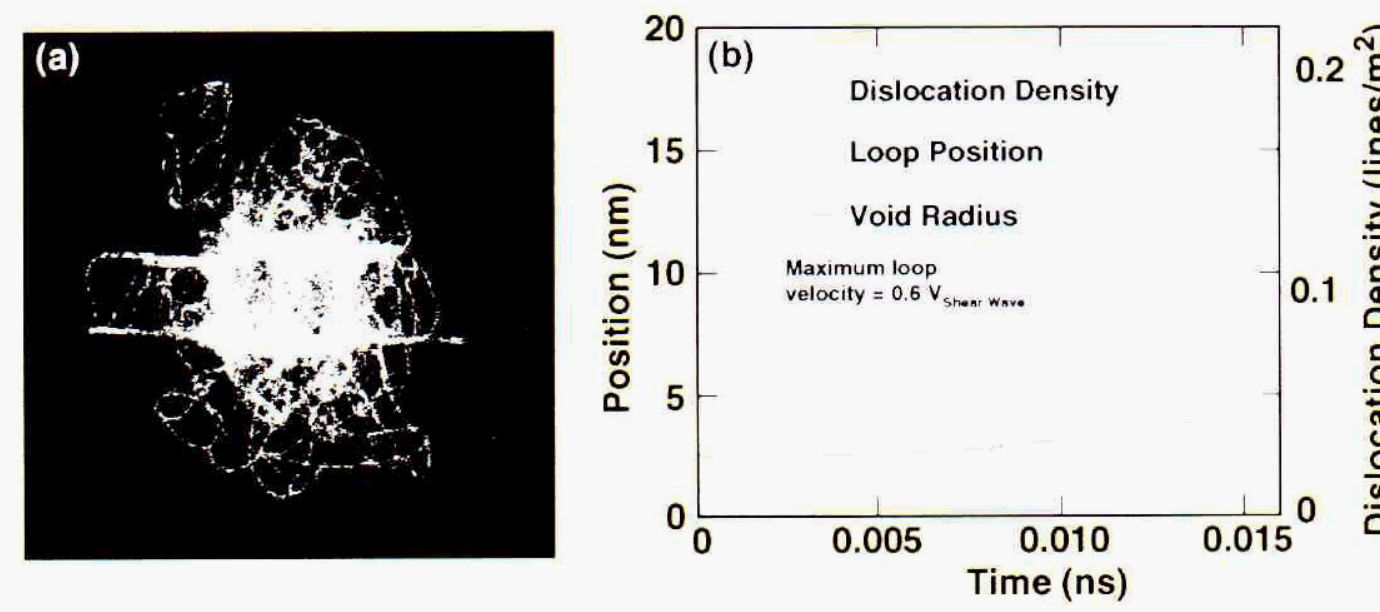

Fig. 10. (a) By showing the positions of only those atoms whose near neighbors are not arranged in the "proper" pattern, dislocations and their motion can be quickly tracked in large-scale molecular-dynamics (MD) simulations. (b) A plot shows the density and average motion of dislocations generated from a void under tensile loading. develop such multiscale models are underpinned by large-scale atomistic simulations of fundamental lattice defects, including dislocations and grain boundaries. In particular, the methods combine quantum-based interatomic potentials with classical molecular-dynamics (MD) simulation techniques to study the generation, motion, and interaction of dislocations at the atomic scale.

This past year, H Division investigators developed new symmetry-based algorithms that allow the rapid identification, for the first time, of dislocations "on the fly" in million-atom MD simulations. (See Fig. 10.) This approach has been used to study in detail how dislocation behavior controls void growth in metals under tensile loading. Information gleaned from these simulations can be utilized in higher-length-scale simulations of dynamic fracture.

\section{NIF 3-omega Solution}

In testimony to the U.S. Senate on April 25, 2001, LLNL Director Bruce Tarter outlined the NIF Programs' major accomplishments. One of the important accomplishments that he noted was an annealing process to increase the damage threshold for the ultraviolet (3-omega) optical elements in the NIF. The process is critical to meeting the NIF's operational requirements.

The initiation and growth of damage sites threatened to destroy the 3-omega optics after only a few tens of shots. Damage to optics occurs because any type of optics polishing is imperfect; imperfections in the polishing leave digs, scratches, and invisible defects on the surface of the optic. The damage sites grow catastrophically after repeated exposure to the powerful ultraviolet laser. However, if the growth of the damage can be mitigated, the optic remains usable. 
Frequent replacement of these optics is a critical factor in the operating cost for the NIF laser. A member of H Division spearheaded a cross-directorate team to address this problem. The team discovered a solution based on localized annealing driven by the use of a carbon dioxide laser. This new technique completely mitigates the growth of damage sites on the surface of the fused-silica optics. The approach is so successful that the NIF Programs Directorate has adopted it and is now in the process of implementing the approach in a production setting.

Figs. 11-13 demonstrate how localized annealing using a carbon dioxide laser successfully mitigated the growth of damage from three different kinds of surface defects.

\section{New Equation-of-State Library}

Last year, H Division built Livermore Equation of State (LEOS), a sophisticated EOS library system, for LLNL Stockpile Stewardship applications. This system consists of the following:

- A global EOS physics package for a very wide range of materials and conditions

- A large library of numerical EOS data with 200 materials and 30 allowed functions per material

- A set of access routines that allows hydrodynamic codes to use EOS data accurately and efficiently

- A browser that allows users to visualize and test stored EOS data.

Access routines now allow simultaneous use of any private or public LEOS library; the LLNL legacy library; and SESAME, the EOS library at LANL. The multi-library system is now the standard version of LEOS. LLNL code developers are finding that the use of the LEOS package for all EOS data increases the efficiency of their codes and allows maximum flexibility in physics models. LEOS is now a critical component of the Stockpile Stewardship Program.
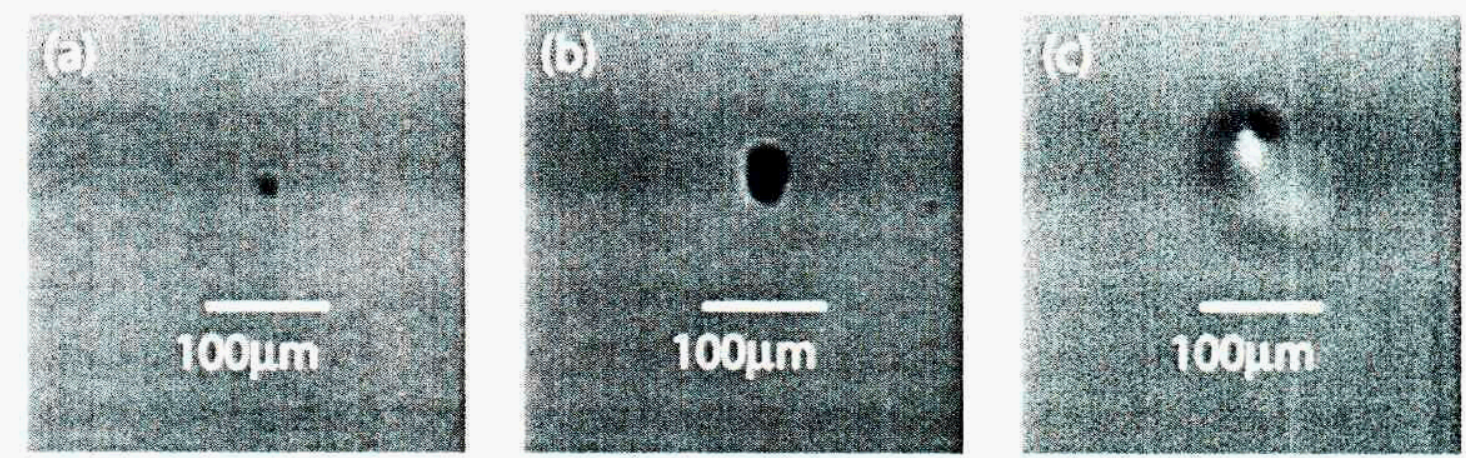

Fig. 11. The use of a carbon dioxide laser mitigated the growth of damage caused by a dig on the surface of a fused-silica optic. (a) Dig precursor before exposure to the 3-omega laser. (b) Damage growth after exposure to $8 \mathrm{~J} / \mathrm{cm}^{2}$ (3-omega at 3 nanoseconds). (c) After carbon dioxide laser mitigation, there was no damage growth after 100 shots at $14 \mathrm{~J} / \mathrm{cm}^{2}$ (3-omega at 3 nanoseconds).
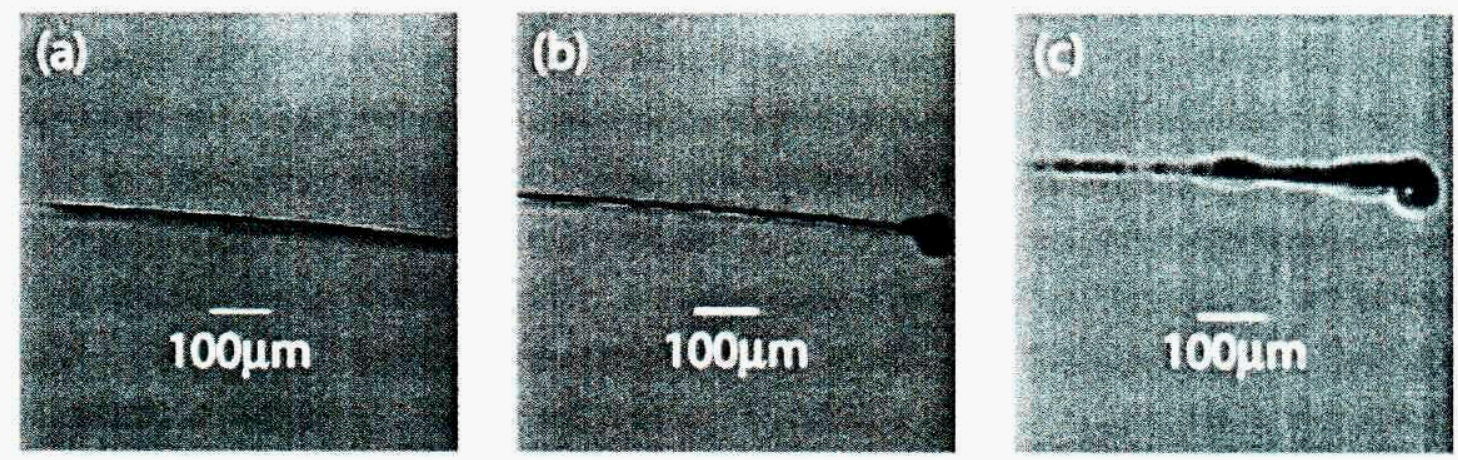

Fig. 12. The use of a carbon dioxide laser mitigated the growth of damage caused by a scratch on the surface of a fused-silica optic. (a) Scratch precursor before exposure to the 3-omega laser. (b) Damage growth after exposure to $8 \mathrm{~J} / \mathrm{cm}^{2}$ (3-omega at 3 nanoseconds). (c) After carbon dioxide laser mitigation, there was no damage growth after 100 shots at $14 \mathrm{~J} / \mathrm{cm}^{2}$ (3-omega at 3 nanoseconds).
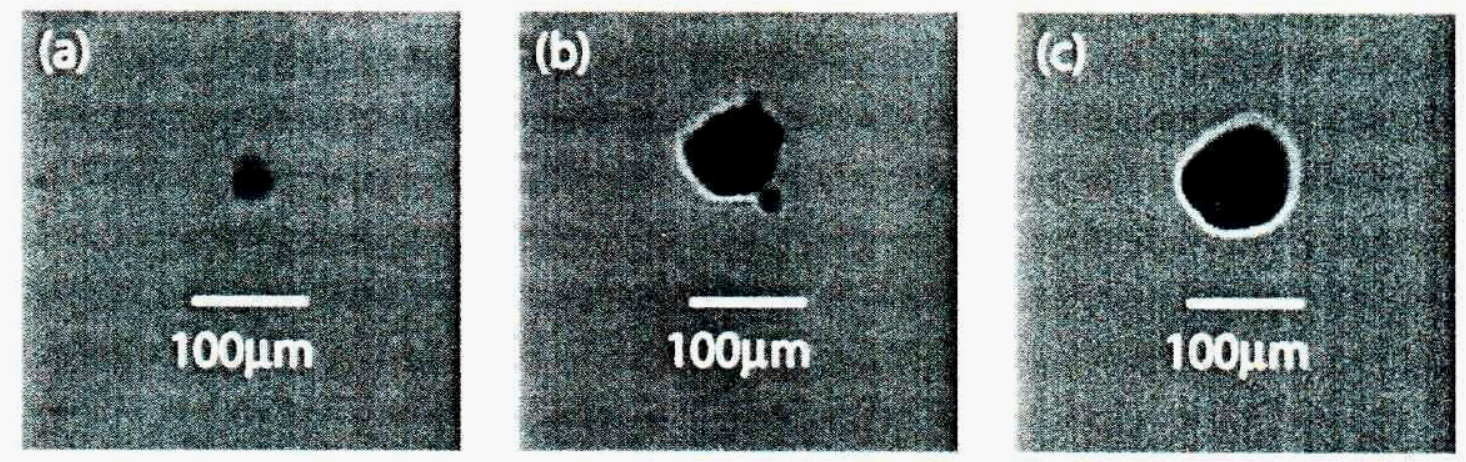

Fig. 13. The use of a carbon dioxide laser mitigated the growth of damage caused by an invisible defect on the surface of a fused-silica optic. (a) Damage growth (no visible precursor) after exposure to $10 \mathrm{~J} / \mathrm{cm}^{2}$ (3-omega at 3 nanoseconds).

(b) Damage growth after exposure to 12 and $14 \mathrm{~J} / \mathrm{cm}^{2}$ (3-omega at 3 nanoseconds). (c) After carbon dioxide laser mitigation, there was no damage growth after 100 shots at $14 \mathrm{~J} / \mathrm{cm}^{2}$ (3-omega at 3 nanoseconds). 


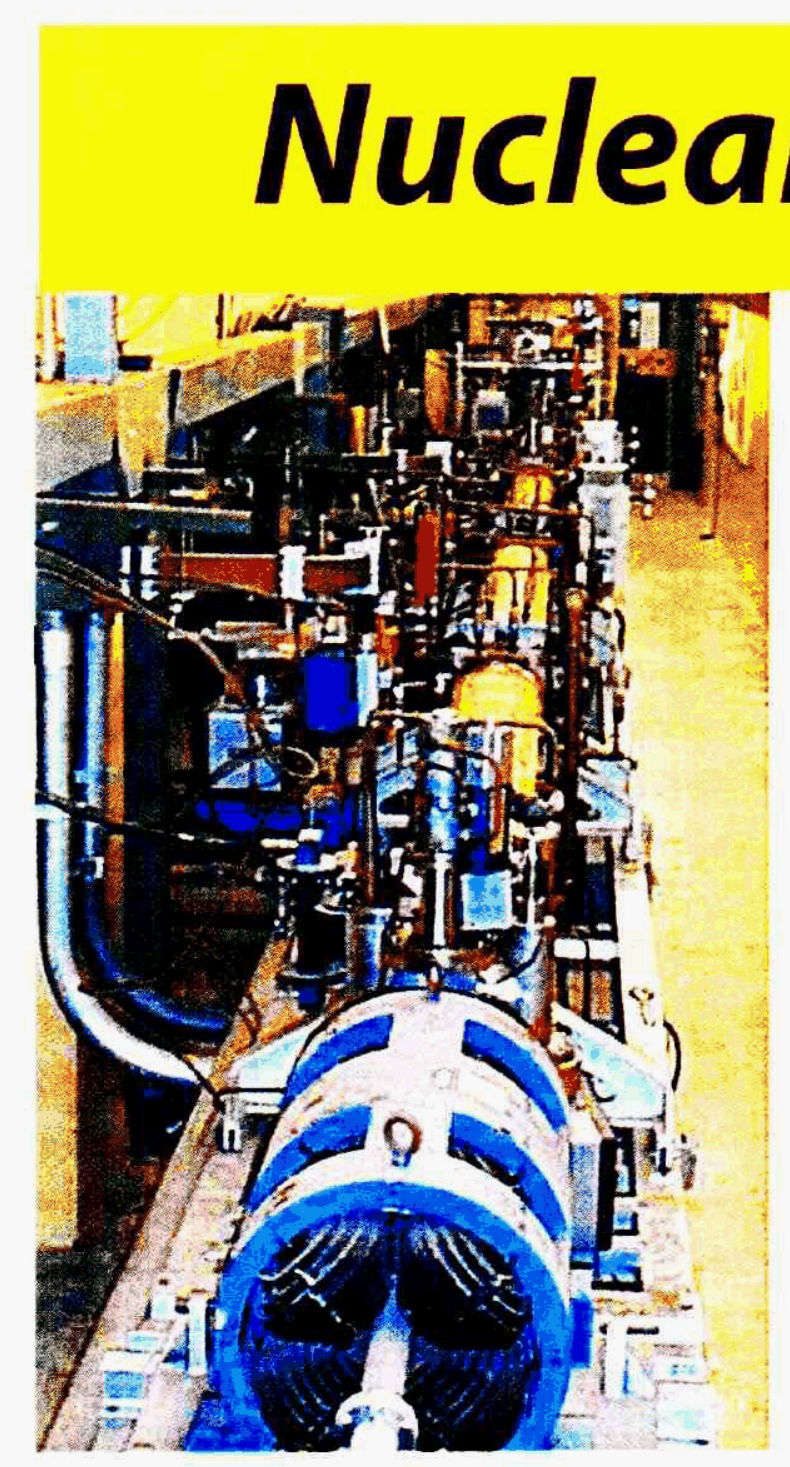

Fig. 14. N Division has built the world's brightest positron source and shortest pulse $x$-ray source for materials research at LLNL's Linac facility.
The Nuclear, Particle, and Accelerator Physics Division (known as N Division) of the PAT Directorate has major activities in experimental and theoretical nuclear physics and experimental high-energy physics. These activities include relevant accelerator and detector technologies, nuclear databases, nuclear physics intensive computer simulations, and experiments on nuclei and elementary particles. In support of the Stockpile Stewardship Program, N Division's primary responsibility is to provide fundamentally derived and fully evaluated nuclear data (such as fission and fusion reaction rates) that are required for the computational simulation of nuclear-weapons performance and tests. The work of N Division is helping to establish the reputation of LLNL as a world-class center of excellence for accelerator science and technology and highenergy physics.

To support these activities, the Division has a strong nuclear theory and modeling effort as well as a strong experimental effort. Current areas of emphasis in the nuclear physics program include measurements of neutron-induced reaction rates, gamma-ray spectroscopy, measurement of radiochemical data required for the analysis of past weapons tests, development of advanced models for nuclear fission, and nuclear-spallation yield determinations.

\section{Development of Nuclear-Physics- Intensive Simulation Code}

N Division is also responsible for developing and maintaining certain nuclearphysics-intensive simulation codes and software. These include 3-D Monte Carlo codes for modeling the transport of ionizing radiation through matter and the development of advanced techniques for Monte Carlo codes. The Division conducts research on particle accelerator and detector technologies that are critical to the design and development of major new facilities. One example is radiography in advanced hydrodynamic tests. In addition, $\mathrm{N}$ Division is involved in accelerator and physics research at the Stanford Linear Accelerator Center (SLAC) near Menlo Park, California; at Brookhaven National Laboratory in Upton, New York; and at the Fermi National Accelerator Laboratory (FNAL) in Batavia, Illinois.

\section{Linac Project}

N Division manages the 100-million-electronvolt linear accelerator (Linac), shown in Fig. 14, that houses the LLNL positron program. This research provides unique capabilities for materials analysis for LLNL programs and basic science investigations as well as industrial uses. In addition, an electron-beam ion-trap program to measure plasma physics spectroscopy has been installed in this facility. The Linac facility also supports a 20-terawatt laser that is used to investigate lasermatter interactions and laser-electron beam physics. In the longer term, a highintensity, 5- to 20-megaelectronvolt neutron capability will be added as well.

Measurement of Critical Cross Section A major goal for the Physical Data Research Program has been a precise (within 10\%) measurement of the ${ }^{239} \mathrm{Pu}(\mathrm{n}, 2 \mathrm{n})^{238} \mathrm{Pu}$ cross section. This cross section is particularly important because the production of plutonium- 238 by neutrons is one of the major diagnostics used in interpreting underground nuclear tests.

Previous measurements of this cross section were performed by counting the number of neutrons emerging from the interaction. This method was extremely difficult and was subject to very large backgrounds. The results of several separate 


\section{ysics Division (N Division)}

experiments and expectations based on nuclear modeling differed by large factors. Neutrons from other reaction channelssuch as $\left(n, n^{\prime}\right),(n, 3 n)$, and $(n, f)$ - form a background to the $(n, 2 n)$ signal channel that makes a precise measurement difficult. The lack of precise knowledge of the small ${ }^{239} \mathrm{Pu}(\mathrm{n}, 2 \mathrm{n})^{238} \mathrm{Pu}$ cross section severely compromises the effectiveness of this important nuclear-test diagnostic.

The experimental nuclear physics group within N Division invented and implemented a new approach to attack this measurement, utilizing its expertise in gamma-ray spectroscopy. The experimental campaign took five years, involved a collaboration with LANL, the construction of a state-of-the-art detector array, and the performance of extremely difficult analyses. This Herculean effort produced important experimental results during this period - the yield of gamma rays as a function of neutron energy. Extracting the ${ }^{239} \mathrm{Pu}(\mathrm{n}, 2 \mathrm{n})^{238} \mathrm{Pu}$ cross section from these results required a combined, intensive effort by the Nuclear Theory and the Computational Nuclear Physics Groups in $\mathrm{N}$ Division. The final results were made available to the design communities at both national laboratories and have caused a reexamination of the archived nuclear test data. For this achievement, LLNL's nuclear physicists received a Weapons Recognition of Excellence Award from DOE Defense Programs. (See the PAT Awards Section.)

\section{Calculation of Nuclei Structure}

N Division's Nuclear Theory Group has embarked on a study to calculate from first principles the structure of nuclei, i.e., how nuclei are put together. This is a fundamentally interesting physics problem because of its complex application of many-body physics with strongly interacting constituents. Understanding the structure of nuclei is important for many reasons:

- Nuclei are a key "laboratory" for testing the fundamental symmetries of nature.

- Nuclear structure dominates the reactions that determine the course of stellar evolution and the synthesis of elements in the universe.

- An improved understanding of nuclei has many important applications to the national security mission of the Laboratory.

Performing these calculations requires the utilization of theoretical ideas from the nuclear shell model and effective interaction theory, along with the exceptional computation power of the ASCI computer facility at LLNL. Initial results showed that the calculation using this approach did converge on a solution. A serious disagreement with the experimentally measured spectrum of ${ }^{10} \mathrm{~B}$ indicated, for the first time, a serious deficiency in the ability of the nucleon-nucleon interaction to describe nuclear structure. The calculations provide compelling evidence that the inclusion of three-nucleon interactions is critical in determining the structure of low-lying nuclear levels. The techniques used in these calculations have many advantages over other methods: they can treat general forms of the nucleonnucleon force, provide a more extensive study of excitation spectrum, and are easier to extend to heavier nuclei. Information gleaned from this work will also help provide answers to questions regarding nuclear structure and reactions of interest to LLNL programs, such as $\left(n, n^{\prime} \gamma\right)$ reactions. 


\section{Uses of Radiographic Probes}

The use of very-high-energy protons as a radiographic probe is being investigated as a technique for an Advanced Hydrodynamics Facility (AHF), which could be a major facility for the Stockpile Stewardship Program. N Division scientists have conducted experiments to measure the fundamental cross sections that would be relevant to an AHF, study the ability of the technique to resolve small features, and investigate the capability to measure the densities of the material traversed by the protons. These crucial measurements will help determine the parameters of the AHF.

In addition, $\mathrm{N}$ Division researchers have been conducting quantitative measurements of the dynamic behavior of materials under shock by using high-energy protons as radiographic probes. Proton radiography has provided dynamic pictures of metals and metal interfaces subjected to high-explosives-driven shocks. The technique has permitted quantitative measurements of the velocity of the shock in the material and of the material ejected from the surface. The precision of the measurements has allowed detailed comparisons with hydrodynamic codes, such as CALE. Future experiments will explore how metals tear or fracture under shock.

N Division leads the Laboratory's program to use low-energy (10-megaelectronvolt) neutrons to conduct radiography and tomography of static objects. The plan is to provide a capability to the plants in the DOE complex (such as Pantex in Texas or the Y-12 facility in Tennessee) so that nondestructive evaluations of weapons systems can be made. This technique can detect cubicmillimeter-sized defects in heavily shielded materials.

\section{High-Energy Particle Research}

$N$ Division leads the Laboratory's involvement in the field of elementary particle or high-energy particle research. Physicists believe a key to the disparity between matter and antimatter in the universe lies in understanding an effect called charge-conjugation-parity (CP) violation. First observed in the 1960s, CP violation refers to the apparently small differences in the way that certain shortlived particles and their antiparticles decay. This violation of a fundamental symmetry may be responsible for the predominance of matter over antimatter in the universe. $N$ Division's intense research effort reached a milestone with its first major publication of results and is at the forefront of inquiry in this field. The work has been enabled by the multiyear construction effort by the SLAC, LBNL, and LLNL team that built the successful SLAC/LLNL/LBNL B Factory at Stanford University.

\section{Next Linear Collider}

$\mathrm{N}$ Division is heavily involved in the design of the Next Linear Collider (NLC), a mammoth accelerator project with a footprint that will be about 30 kilometers (20 miles) long and that could cost more than $\$ 5$ billion. The NLC will house two opposing linear accelerators inside a 30-kilometer tunnel.

When completed, the NLC will accelerate fundamental particles, the building blocks of the universe, to energies in the teraelectronvolt range-i.e., the equivalent of a trillion electronvolts. The NLC will probe the origins of mass, the structure of spacetime, and dark matter. $N$ Division has been instrumental in developing plans to backscatter laser light from the electron beams in the NLC, thereby opening the possibility of studying the collisions between extremely high-energy gamma rays. The development of this "gammagamma collider" option utilizes the unique strengths of LLNL in addressing an extremely challenging investigation.

\section{Linac Coherent Light Source}

$N$ Division is involved in the design of the Linac Coherent Light Source, a fourthgeneration $x$-ray light source. This light source at the SLAC is expected to produce 
$x$-ray beams more than ten orders of magnitude brighter than existing sources, requiring the development of new approaches to x-ray optics.

\section{Pioneering Electron Ion Experiment}

$N$ Division is involved in experimental programs at Brookhaven National Laboratory's Relativistic Heavy-Ion Collider (RHIC), searching for quark-gluon plasmas, and at the FNAL, measuring particle production in proton-nucleus collisions and searching for neutrino oscillations.

The Pioneering Electron New Heavy Ion Experiment (PHENIX) is a multifaceted heavy-ion experiment at the RHIC. LLNL designed much of the PHENIX magnet system and supervised its fabrication and testing. The system consists of a central magnet and two flanking Muon magnets, and plates of Muon Identifier steel that sandwich the Muon magnets. The entire systems stands more than three stories tall and weighs in excess of 2,200 tons (see Fig. 15).

This highly collaborative project is being used in the search for elusive quarkgluon plasma, a form of matter believed to hold subatomic matter together. The collider recreates conditions that were present approximately 1 microsecond after the Big Bang. It has been predicted that the high-energy densities created in these collisions will precipitate a phase transition from Quantum chromoDynamics (QCD) bound states (protons, neutrons, pions, etc.) into a plasma of quarks and gluons.

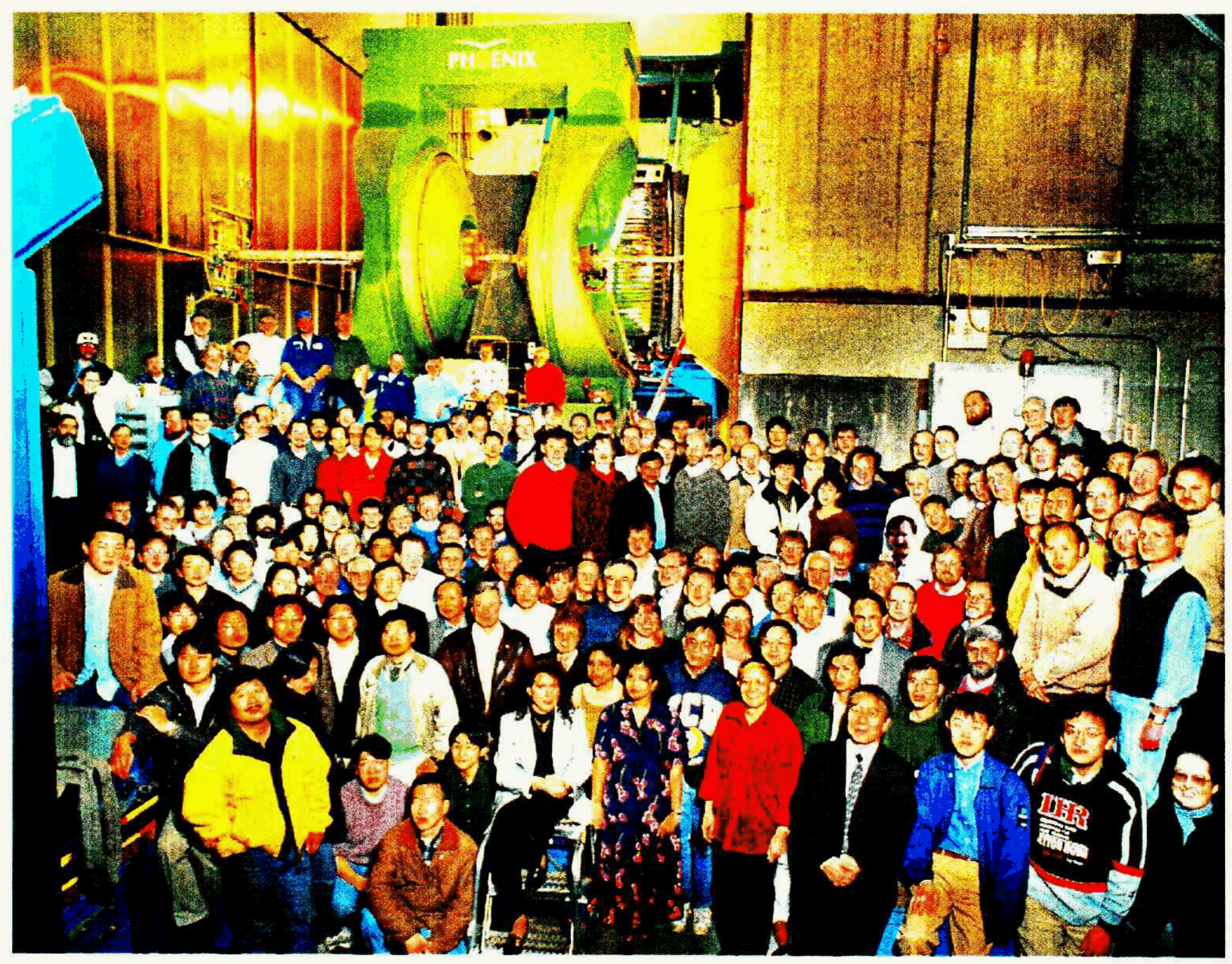

Fig 15. The PHENIX collaborators assembled in front of the LLNL-designed central magnet at the Relativistic Heavy lon Collider (RHIC) sited at Brookhaven National Laboratory. Strong magnetic fields are used to bend the trajectories of elementary particles produced in the collision so that they may be identified by their curvature. The experiment is designed to search for and study new forms of nuclear matter by recreating conditions in the first microsecond after the Big Bang. Collaborators in PAT are studying the source size of these collisions by measuring correlations between pairs of identical particles. PAT group members included in the photograph are Ron Soltz, Ed Hartouni, Stephen Johnson, and Jane Burward-Hoy. Mike Heffner also works on this project. 


\section{Fusion Energy Program}

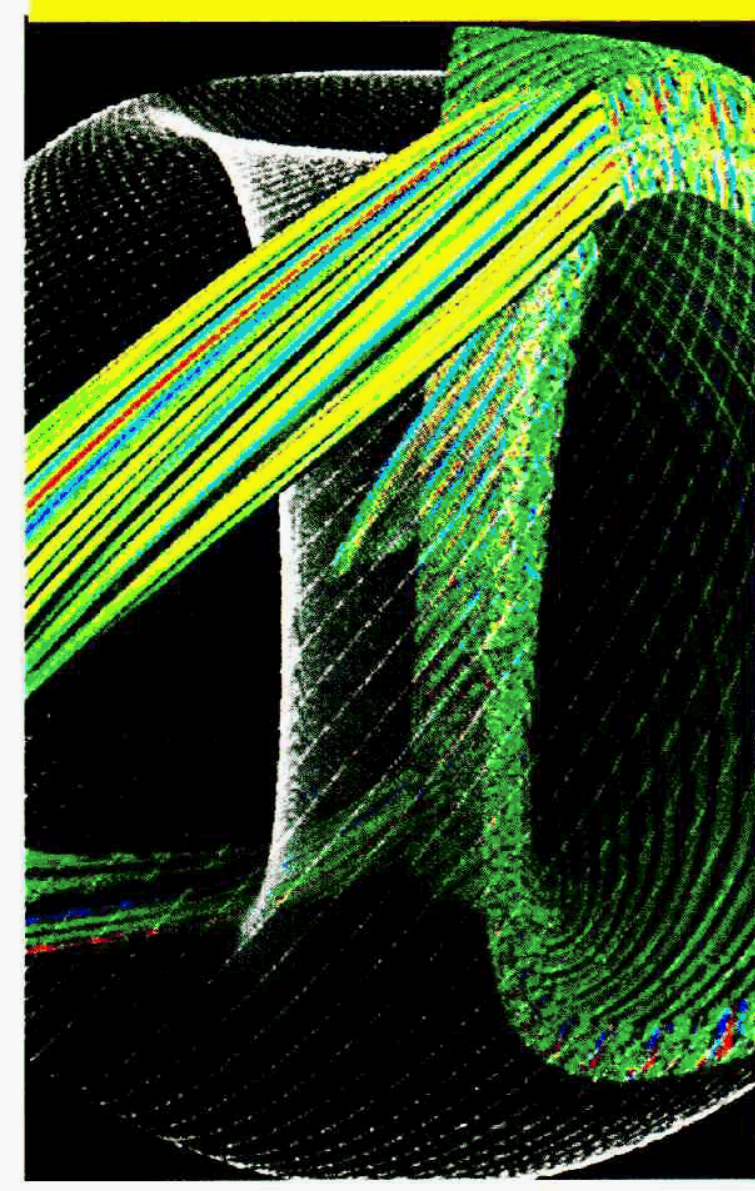

This computer simulation, relevant to magnetic fusion energy research, shows a magnetic field line (white) wrapping around a torus, or doughnut-shaped configuration of plasma. Magnetic field lines are embedded within the plasma, with individual particles traveling along each field line. The color contours indicate microturbulent fluctuations in the plasma density. Regions with similar density microturbulent eddies are indicated by regions of similar color stretch along the field lines, while varying rapidly across the field lines. These microturbulent eddies transport heat from the plasma's superhot core to the cold outer edge.
The Fusion Energy Program (FEP) consists of three subprograms: Magnetic Fusion Energy (MFE), Inertial Fusion Energy (IFE), and the Beam Research Program. MFE and IFE are closely related in their goal to develop fusion energy, and the two subprograms share a combined technology program and several staff members who work in both IFE and MFE. The Beam Research Program provides advanced linear induction accelerator development for radiography and other applications, including IFE. (Note that programmatic responsibility for the nonIFE work is in the Defense and Nuclear Technologies [DNT] Directorate and is not covered here.) Described below are the accomplishments and goals of the MFE and the IFE subprograms.

\section{Magnetic Fusion Energy Theory Program}

The MFE Theory Program contributes significantly to the:

- Study of plasma turbulence

- Physics of the plasma edge (where toroidally confined fusion plasmas interface with the confinement vessel)

- Physics of confinement concepts considered as alternatives to the tokamak

- Integrated modeling codes important to magnetic fusion experiments.

The gyrokinetic simulation codes developed at LLNL have been the standard benchmark for other major kinetic and fluid codes that describe plasma turbulence in the core of confinement devices. Fig. 16 shows a simulation of turbulent structures responsible for transporting energy from the core of a tokamak plasma. In this simulation (and as seen in experiments), large, coherent structures responsible for transport are disrupted by shear in the plasma flow at late times.
Occupying a unique position because of the completeness of its physics and geometry, the BOUT 3-D edge-turbulence code is in increasing demand by researchers worldwide. The two-dimensional (2-D) edge-fluid code, UEDGE, is the principal edge-modeling tool in the U.S. program and is well known (along with its principal author) worldwide. The unique capabilities of the integrated modeling code, CORSICA, make it a valued design tool for work with the International Thermonuclear Experimental Reactor (ITER) and, more recently, a design and analysis tool for spheromaks and reversed-field pinches (RFPs). LLNL and its collaborator, General Atomics (GA), have combined CORSICA with the GA code ONETWO to provide a combined analysis and predictive code called CALTRANS for the DIII-D tokamak at GA. Recently,

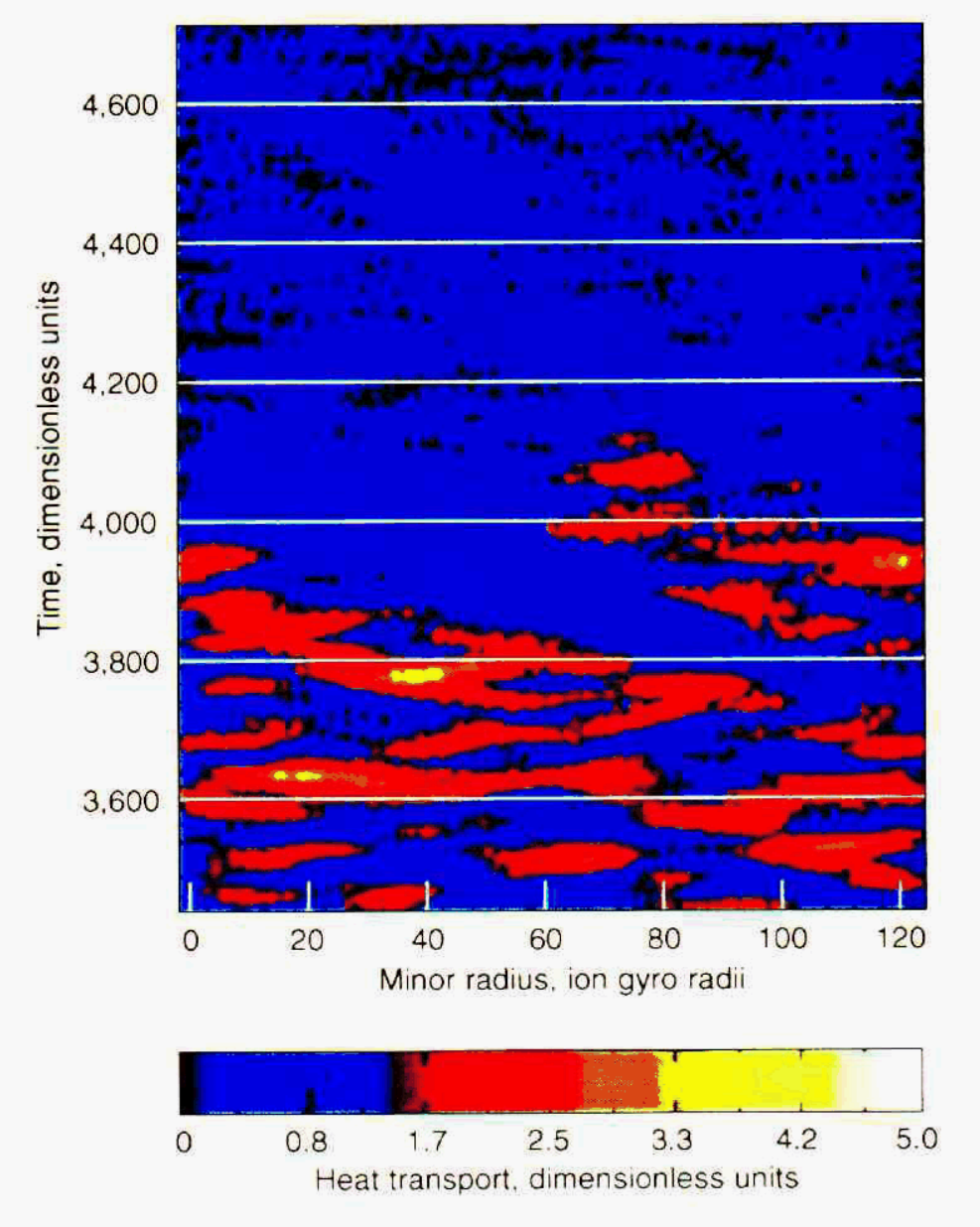

Fig. 16. Large, coherent structures responsible for energy transport are disrupted by shear in the plasma flow, as seen in experiments. 
CALTRANS was applied for the first time in electron-cyclotron heating experiments. It has also provided key building blocks and concepts for the National Transport Code initiative.

\section{DIII-D Collaboration Program}

LLNL has an ongoing collaborative research program on the DIII-D National Fusion Facility (shown in Fig. 17) in San Diego, California. This collaborative effort is an integral part of the overall program to improve the tokamak concept, which has been used to advance the science of high-temperature plasmas in magnetic fusion research. In this collaboration, FEP plays a leading role, providing scientific, operational, and management support for the experiment. (FEP supports the development and operation of DIII-D but does not have the primary responsibility for the facility.)

LLNL's focus in DIII-D work is in two main areas. First, FEP is responsible for the measurement and the modeling of the physical processes important for heat and particle control in the edge plasma and divertor. FEP has designed and now operates state-of-the-art plasma diagnostics, including:

- Divertor Thomson scattering (Still the only such system on a tokamak divertor, it verified divertor plasma temperatures in the 1-electronvolt range.)

- Infrared thermography (The world's most comprehensive system, it is used to directly measure the heat flux to surfaces.)

- 2-D, visible, and extreme ultraviolet (EUV) spectroscopy (The only EUV system and the most comprehensive visible system, it is used to measure the distribution of impurities and radiation in the divertor.)

- Development of higher time-resolution diagnostics to measure fast-divertor phenomena, such as Edge Localized Modes (ELMs).

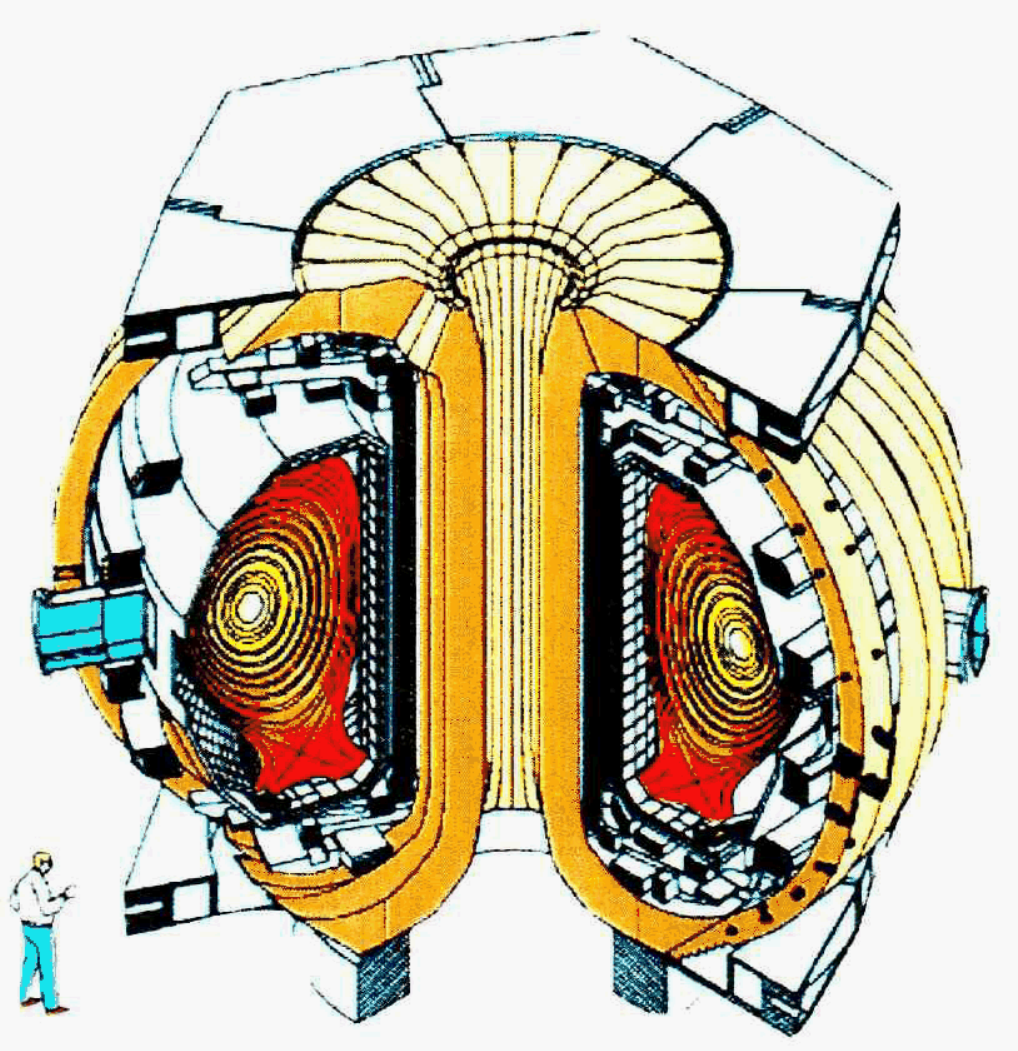

Fig. 17. LLNL has a significant role in the DIII-D Tokamak, which is a vital part of the world fusion science program. Work on the DIII-D Tokamak has involved the following international collaborators: industrial partners $\mathrm{CPI}$, Gycom,

CompX, Orincon, Creare, Thermacore, IR\&T, Surmet, HiTech Metallurgical, TSI

Research, and FAR Tech; European partners Joint EuropeanoTrus, IPP, and KPA (Germany), Cadarache (France),

The LLNL UEDGE code has been benchmarked with experimental data, resulting in the identification of important physical processes, such as plasma flows. It has become the standard edge computational tool in the U.S. fusion program and is being applied to other magnetic configurations, such as stellarators and other shaped tokamaks (C-MOD at the Massachusetts Institute of Technology and KSTAR in Korea). Both the experiments and the modeling have examined the role of plasma and divertor shape on the overall effectiveness of the divertor.

The second LLNL area of responsibility on DIII-D is the measurement and the modeling of the plasma-current profile. LLNL has developed a 38-channel Motional Stark Effect (MSE) diagnostic. The MSE diagnostic instrument provides the world's best measurements of plasmacurrent profiles and electric-field profiles required to understand the MHD stability properties of the tokamak plasma. In 2001, the DIII-D experiments focused on controlling these profiles with a localized electron current drive - a key part of the tokamak advancement goals of the DIII-D program.
Culham (England),

Lausanne

(Switzerland), FOM

(Holland), and Frascati (Italy); Japanese partners JAERI, JT-60U, JFT-

2M, NIFS, LHD, and

Tsukuba University;

Russia partners

Kurchatov, Troitsk, loffe, Keldysh, and

Moscow State; and other international partners, including KAIST and KBSI from Korea; CCFM, University of Alberta, and University of Toronto (Canada); Chalmers University (Sweden); Helsinki University (Finland); University of Wales (Wales); and SWIP, ASIPP, SINICA, and Southwest Instr. (China). 


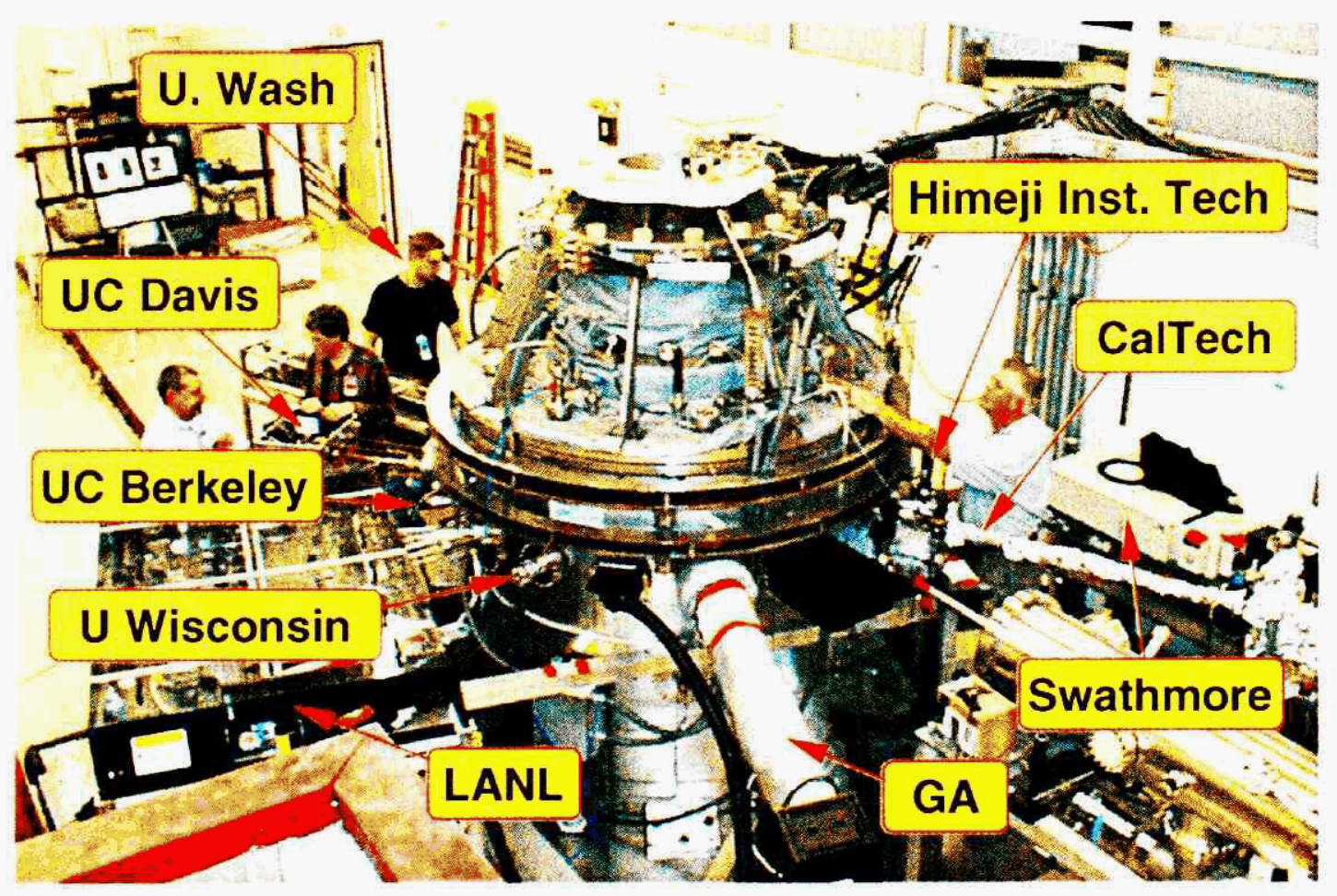

Fig. 18. The Sustained Spheromak Physics Experiment (SSPX) is now in its third year of operation. The labels indicate the role of various institutions in the LLNL-led effort.

Fig. 19. The 500-kilovolt ion-source test stand at LLNL was completed at the end of 2001.

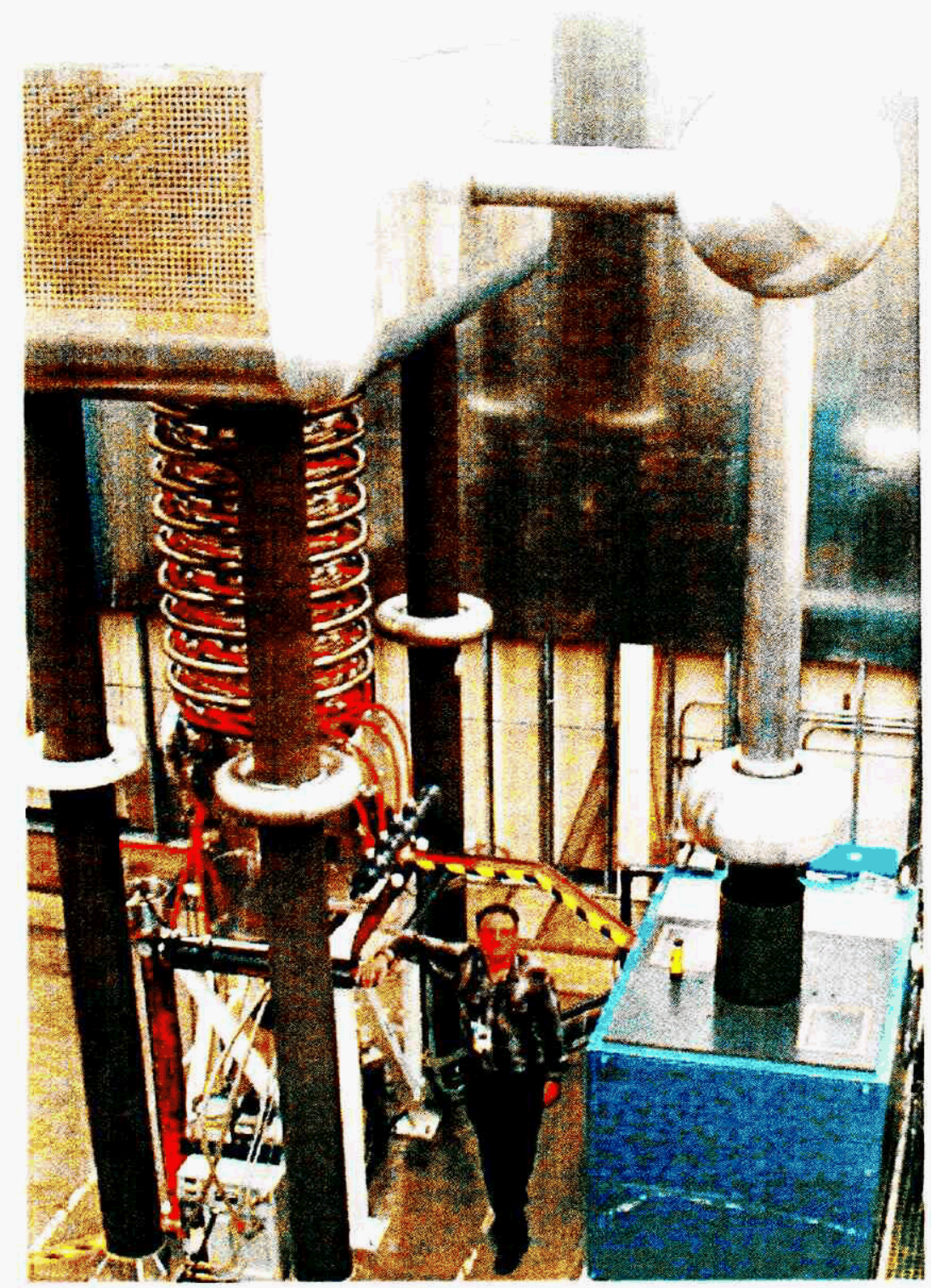

The Sustained Spheromak Physics Experiment (SSPX), shown in Fig. 18, explores spheromak formation and sustainment. The spheromak, an advanced and alternate plasma-confinement concept, has an internal dynamo to create its confining magnetic field.

Installation of a Profile Thomson Scattering Diagnostic has been completed, and now electron temperature data are routinely obtained for use in determining the core energy confinement time. SSPX is the first of the new set of alternate concept devices in the National MFE Program to have a working-profile Thomson scattering system. New magnetic field configurations can be explored with unprecedented flexibility, compared to other spheromaks.

The SSPX device is the lead spheromak experiment funded by DOE's Office of Fusion Energy Sciences (OFES) and is one of the larger efforts within the U.S. Alternate Concepts Program. The OFES has called the SSPX a

\section{Spheromak Program}

"world-class facility." Recent experiments indicate that temperatures of 150 electronvolts have been achieved in this device.

\section{Heavy-Ion-Fusion Virtual National Laboratory Program}

Initiated in 1998, the Heavy-Ion-Fusion Virtual National Laboratory (HIF-VNL) is a coordinated IFE research program with LLNL, LBNL, and the Princeton Plasma Physics Laboratory (PPRL) working together as partners - combining human resources, budgets, and experimental facilities to maximize progress toward common HIF development tasks. As a result of this VNL agreement, LLNL and LBNL have successfully combined resources to develop three new beam experiments: a high-current transport experiment (HCX) and a neutralizedtransport experiment (NTX) at LBNL, and a 500-kilovolt ion-source development experiment at LLNL (Fig. 19).

Construction of the 500-kilovolt ionsource test stand was completed in 2001.

Central to the success of the VNL experiment is the teamwork among mixed groups of experimentalists and theorists from the VNL partners. Of the total HIF-VNL resources and staff, more than one-third are from LLNL. A key element of the LLNL effort in the HIF-VNL is ion-beam modeling. The LLNL beam-theory and simulation group is widely recognized as outstanding and unique in the field. Fig. 20 shows some of the group's representative modeling predictions.

\section{IFE Target Design Program}

LLNL's preeminent IFE design group relies on the large investment made by DOE Defense Programs in providing very capable computational tools. These tools were benchmarked in an extensive experimental program culminating in the Nova laser technical contract. This continuing support from DOE Defense Programs allows IFE to be both broad (designing all types of IFE targets) and deep (calculating target performance from incident energy deposition through thermonuclear burn to the insult the target applies to the reaction chamber for some target types). 
IFE continues to invent new targets and variations of older designs. For example, Fast Ignition (FI), an LLNL invention, is now established as a significant experimental and theoretical research effort in four other nations. LLNL scientists invented the distributed radiator target

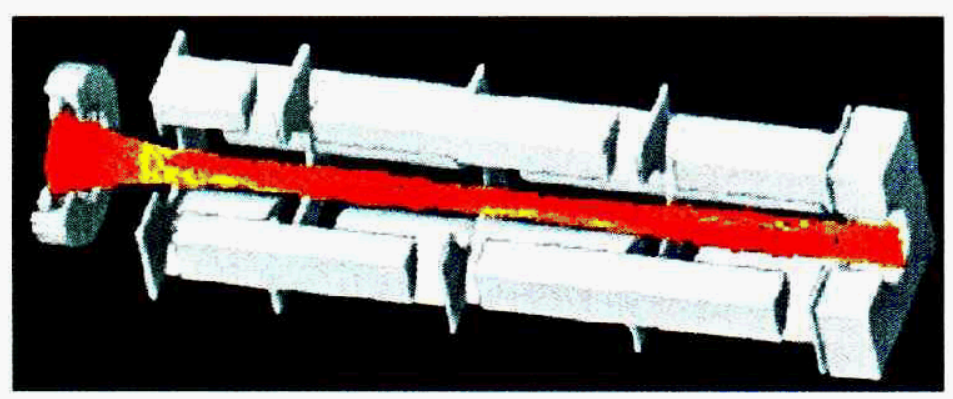

Acceleration in 3-D structure

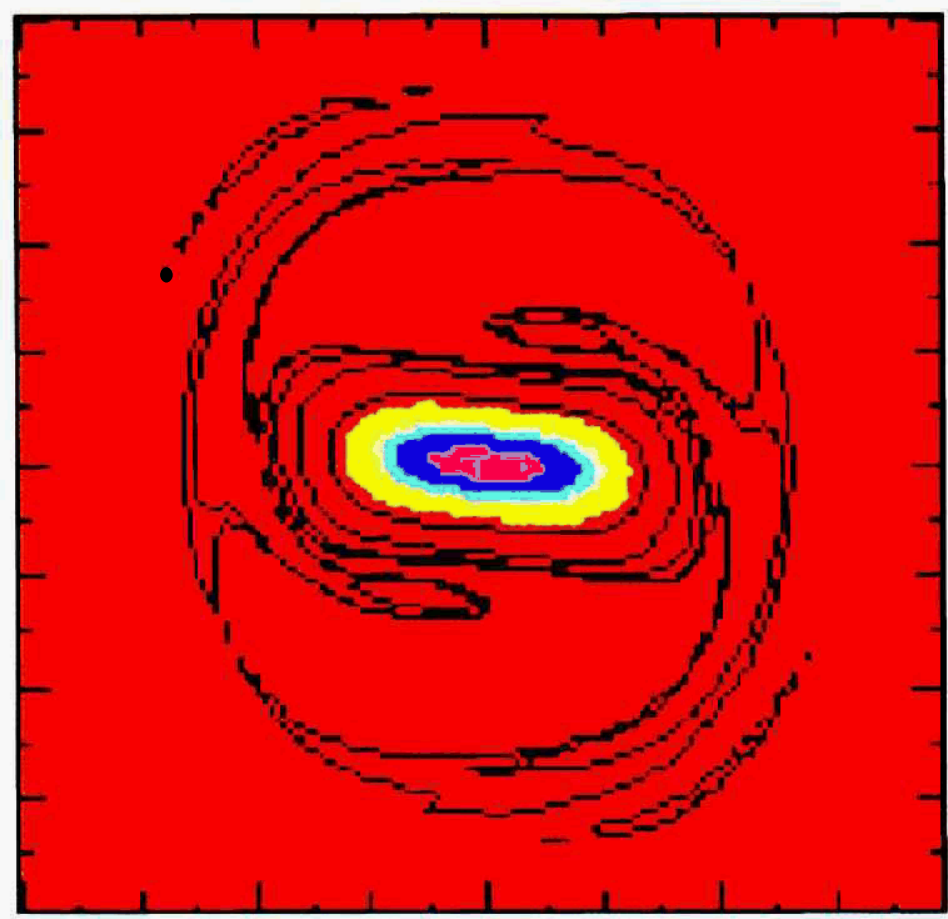

Halo generation

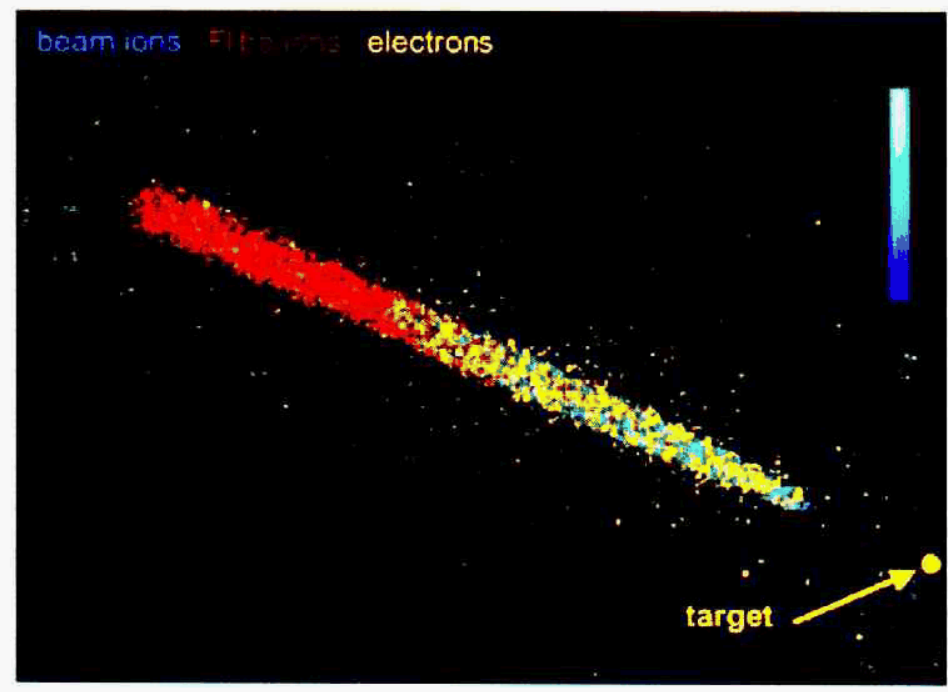

Beam propagation in chamber

Fig. 20. LLNL has a central role in ion-beam modeling for the heavy-ion fusion energy program. for heavy-ion fusion, and LLNL was the only laboratory in the world that successfully carried out integrated calculations from energy deposition through burn for any heavy-ion design using two-sided illumination. (Two-sided illumination is required for thick-liquid protection of fusion chamber first walls-a key idea for relaxing the fusion materials requirements.)

In the past year, LLNL researchers progressed on a number of fronts, including the use of larger beam-spot areas. As indicated in Fig. 21, the focalspot requirements on the ion-beam accelerator can be varied by choosing different approaches to achieving capsule symmetry. A target design for heavy-ion fusion was developed that increases the allowed beam-spot area (relaxing beamfocusing requirements) by almost a factor of 3 . Researchers obtained a simple formula that describes the one-dimensional ignition threshold for all hot-spotignited capsules of interest to IFE.

Target physics and design are essential components of all approaches to IFE. Historically, target design has set the highlevel requirements for the other program elements because of the centrality of achieving ignition and high gain for this approach to energy. Target design sets the driver energy scale and characteristics (such as particle energy, power, pulse shape, focusing requirements, illumination geometry, and power balance).

Target design also defines the target materials and fabrication finish levels. Innovations in target design can translate into significant relaxation of requirements for the other program elements. For example, IFE recently designed a capsule that required an order of magnitude less ion-beam energy than previous reactor capsule designs. This sort of leverage makes target design an essential component of the program. 


\section{Fast Ignition Project}

New in Fiscal Year 2000-2001 was a fouryear concept exploration program funded by the OFES in Fast Ignition (FI) inertial fusion. FI relies on conventional compression of fusion fuel, followed by ignition with a short-pulse, high-power beam. The key unresolved scientific issue in FI is the efficiency of energy transfer from a shortpulse laser beam to the ignition hot spot via relativistic electrons created in the absorption of laser radiation. The science of the relativistic laser matter interaction and of electron-beam propagation in dense plasma at extreme current densities is the subject of intense interest worldwide both for its intrinsic novelty and its wide range of potential applications. The project is highly collaborative, linking universities (Princeton University and the University of California campuses at Davis and at San Diego) and laboratories (LLNL and

\section{Original distributed radiator target}

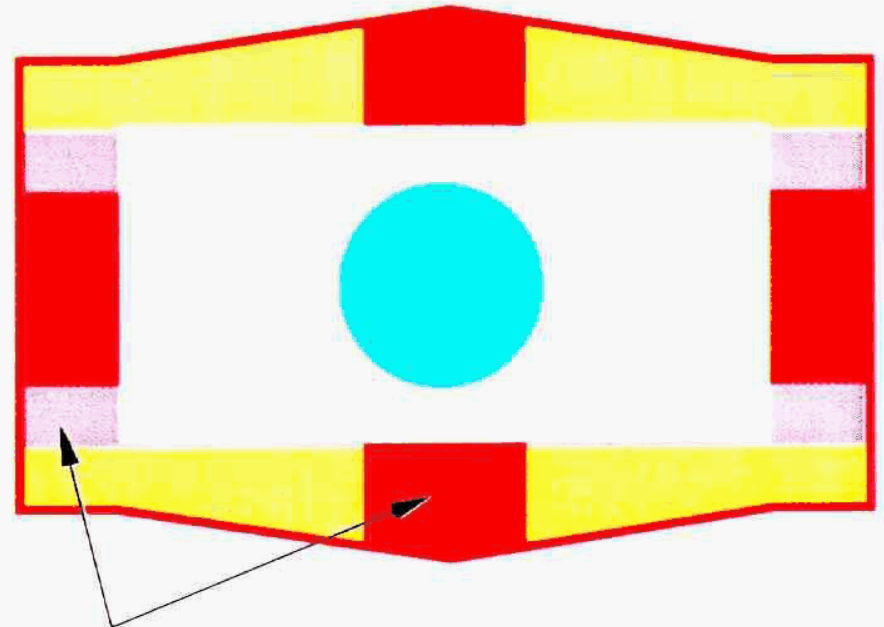

Pressure balance holds position of radiators
(iA) in the United States with international research teams at the world's leading high-intensity laser facilities in Japan, the United Kingdom, and France. The project team has strong relevant experience gained through three years of experiments and related modeling at the LLNL Nova Petawatt Laser, which was decommissioned in 1999.

The motivation for this project is the potential of FI for high-energy gain and reduced driver constraints, both of which make FI more attractive than conventional Inertial Confinement Fusion (ICF) ignition for an IFE power plant. The goal is to use experiments and modeling to demonstrate the feasibility of full-scale FI, thereby justifying a transition to proof-ofprinciple scale experiments. A future possibility is to capitalize on interest in the application of ultrapowerful and

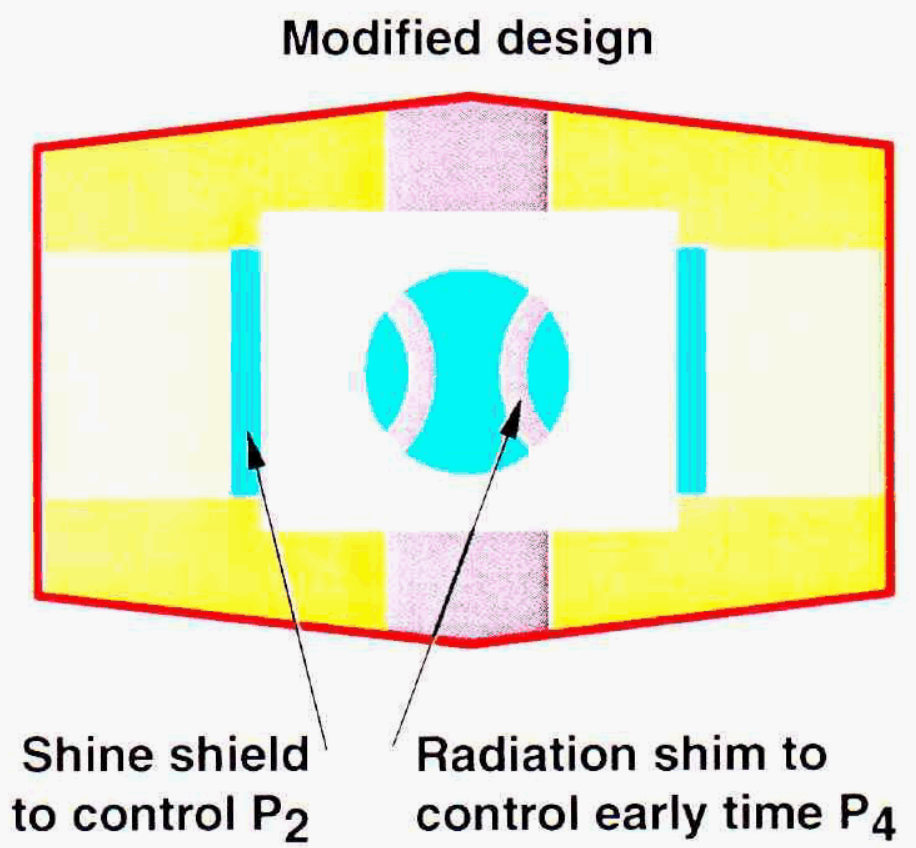

Beam spot: $1.8 \mathrm{~mm} \times 4.1 \mathrm{~mm}$

Effective radius: $2.7 \mathrm{~mm}$

Beam energy: $5.9 \mathrm{MJ}$

Gain $=68$

\section{Beam spot: $3.8 \mathrm{~mm} \times 5.4 \mathrm{~mm}$ Effective radius: $4.5 \mathrm{~mm}$ Beam energy: $6.7 \mathrm{MJ}$ Gain $=58$}

Fig. 21. Under exploration are symmetry-control techniques to allow larger beam spots for distributed radiator targets for heavy-ion fusion. A $66 \%$ increase in beam radius resulted in a $14 \%$ increase in beam energy. 
intense lasers in DOE's OFES and Defense Programs, as well as in the National Science Foundation and the National Aeronautics and Space Administration, to obtain shared funding for a center of excellence.

\section{LLNL Fusion Technology Program}

The LLNL Fusion Technology Program includes activities in both MFE and IFE. The OFES manages its enabling technology research and development through the Virtual Laboratory for Technology (VLT). The VLT is organized into five major groups: Plasma Technologies, Fusion Technologies, Materials, Advanced Studies, and IFE Technologies. LLNL currently has funded activities in all five categories.

LLNL applies its expertise in a variety of technology areas within the VLT.

Researchers involved in these programs are recognized experts and leaders in their respective areas. LLNL's work in the Plasma Technologies area includes the design and testing of magnets for MFE. One LLNL scientist is the international test group leader for the (ITER) Central Solenoid Model Coil. The same group also provides innovative magnetic design and testing for the quadrupole-focusing magnets for heavy-ion drivers. As part of the Plasma Facing Components program of Plasma Technologies, LLNL's work on edge plasmas (the UEDGE code) is applied to shielding the core plasma from liquid-wall vapor.

LLNL is involved in the Advanced Power Exchange (APEX) program (part of the Fusion Technologies element of the VLT), where researchers are looking at the application of thin- and thick-liquid walls for MFE chambers. In addition to working on concept design and fluid-flow considerations, LLNL has applied its benchmarked UEDGE fluid code to predict divertor and edge-plasma behavior for these chambers. The UEDGE work is an excellent example of the application of science-based, first-principles modeling to fusion technology issues.

LLNL also has unique and growing capabilities in the area of neutron damage modeling. LLNL's excellence in this area has been recognized by its increased role in the OFES's Fusion Materials Program, which was awarded through a competitive, peer-review process. The goal of the LLNL materials modeling project is to apply predictive, physically based, multiscale modeling to the study of the underlying mechanisms responsible for materials degradation in the fusion environment. The ultimate objective of the project is to develop a modeling capability to aid advanced materials design. This is important as the OFES restructures the advanced materials program to focus on the underlying science required to develop materials for the fusion power environment.

In IFE, LLNL is centrally involved in the design and integration for thick-liquidwall chambers, in driver/chamber interface design, in safety and environment modeling and assessment, and in powerplant systems modeling and analysis. 


\section{Institute of Geophysics and Planetar}

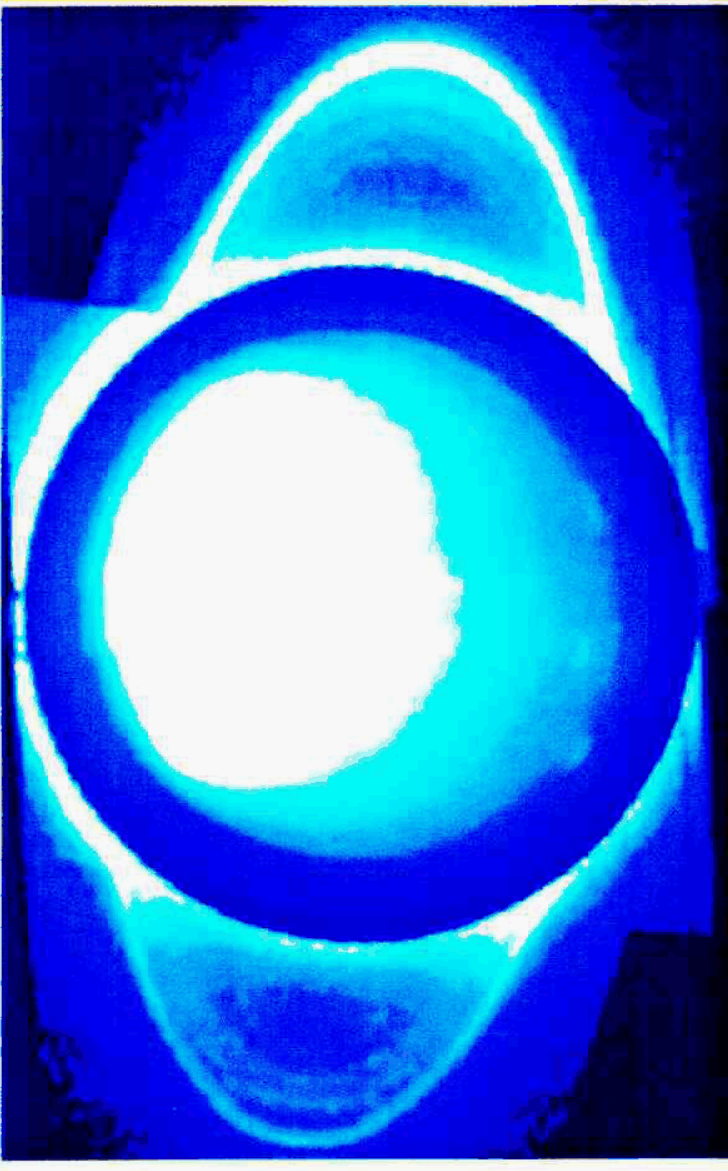

Fig. 22. The use of adaptive optics at the Keck Observatory resulted in the first ground-based detection of the inner rings of Uranus.
The Institute of Geophysics and Planetary Physics (IGPP) was founded in 1946 at the University of California at Los Angeles with a charter to "promote and coordinate basic research on the understanding of the origin, structure, and evolution of the Earth, the Solar System, and the Universe, and on the prediction of future changes, as they affect human life." As a Multicampus Research Unit of the University of California system, IGPP now has branches at University of California campuses in Los Angeles, San Diego, Riverside, and Santa Cruz, and at LLNL and LANL. Because it is University-wide, IGPP has played an important role in establishing interdisciplinary research in the earth and planetary sciences.

Each of the six branches has a somewhat different scientific emphasis, reflecting the strengths of campus departments and Laboratory programs. The IGPP branch at LLNL emphasizes research in tectonics and geochemistry in its Geosciences Center, and planetary science and astrophysics in its Astrophysics Center. It provides a venue for studying the fundamental aspects of these fields, thereby complementing other LLNL programs that pursue applications of these disciplines in national security and energy research.

The IGPP branch at LLNL, along with the branch at LANL, sponsors the University Collaborative Research Program (UCRP) to facilitate scientific collaborations among researchers at the University of California campuses and those at the national laboratories in areas related to earth science, planetary science, and astrophysics. This program provides funds for joint research projects among University of California researchers, other academic research centers, and LLNL. The goal of the UCRP is to enrich research opportunities for University of California scientists by making available to them some of LLNL's unique facilities and expertise and by broadening the scientific program at LLNL through collaborative or interdisciplinary work with campus researchers.

Although the permanent LLNL staff assigned to IGPP is relatively small (at present about seven full-time employees), IGPP's research centers have become vital research organizations through its:

- Support of a substantial number of resident postdoctoral fellows

- Funding of 20 or more UCRP projects each year

- Hosting of a variety of visitors, guests, and faculty members from many institutions

- Hosting of conferences, workshops, and seminars in astrophysics and geosciences.

IGPP's Astrophysics Research Center (ARC) delivers cutting-edge science and technology in the pursuit of basic astrophysical knowledge. The ARC focuses on astronomical adaptive optics, timedomain astronomical surveys, radio surveys, and the formation and evolution of structures in the early universe. These projects draw upon and enhance LLNL capabilities in advanced detectors, remote sensing, advanced scientific computing, high-energy-density physics, and highenergy physics. This leading-edge science has generated positive impressions in the scientific community. The technology of adaptive optics, the incredible adaptive optics images (surpassing those from the Hubble Space Telescope), the discovery of forming galaxies in the very early universe, and the creation of the field of microlensing surveys have all been featured in newspaper articles and the popular scientific press. Last year, ARC scientists won more than 150 nights of major telescope time, more than 100 orbits of Hubble Space Telescope time, 


\section{y Physics}

significant time on a variety of other satellites, and hundreds of hours of Very Large Array (VLA) radio telescope time.

\section{Astronomical Adaptive Optics}

Essential to astronomers today are optical systems that correct atmospheric aberrations for incoming light. Known as adaptive optics, these system can be mounted on a telescope and directed into virtually any part of the heavens that an astronomer wants to study. In adaptive optics, mirror adjustments remove Earth's atmospheric turbulence from the telescope's images, thereby producing unprecedented clarity. The prototype adaptive optics system was installed at the University of California's Lick Observatory on Mount Hamilton near San Jose. LLNL researchers then helped develop and install a larger adaptive optics system at the Keck Observatory atop Mauna Kea in Hawaii.

IGPP uses the adaptive optics system in three major scientific areas. The first is in planetary science. LLNL researchers, in collaboration with researchers at the University of California at Berkeley are using the Keck adaptive optics system to obtain spatially resolved spectra of Titan, Io, Neptune, and Uranus. Highlights of this work include detection of an extremely powerful volcanic outburst on Io, the first ground-based detection of the faint inner rings of Uranus (Fig. 22), and completion of an absolute reflectance map of Titan.

The second area consists of studies of nearby active galaxies, including spectroscopic mapping of the merging nuclei of NGC6240 at 0.04-arc-seconds resolution.

The third area is the search for extrasolar planets. In collaboration with scientists at the University of California at Los

Angeles, IGPP researchers are using adaptive optics to search for planetary companions to young stars. The use of the Keck adaptive optics system permits the detection of companions 1 million times fainter than their parent star, sufficient to detect a 10-million-year-old Jupiter-mass planet through its thermal emission in the near-infrared wavelength. IGPP is also mapping out the Trapezium star-forming region in Orion to study the environments of young stars. Shown on the front cover is the laser guide star adaptive optics system in use at the Keck Observatory.

The LLNL system at the Lick Observatory is the only sodium laser guide star currently in operation. LLNL's 20-watt dye laser produces an eighth-magnitude artificial star. It is routinely achieving Strehl ratios of 0.5 - to 0.15 -arc-seconds resolution. A similar laser has been integrated into the Keck adaptive optics system.

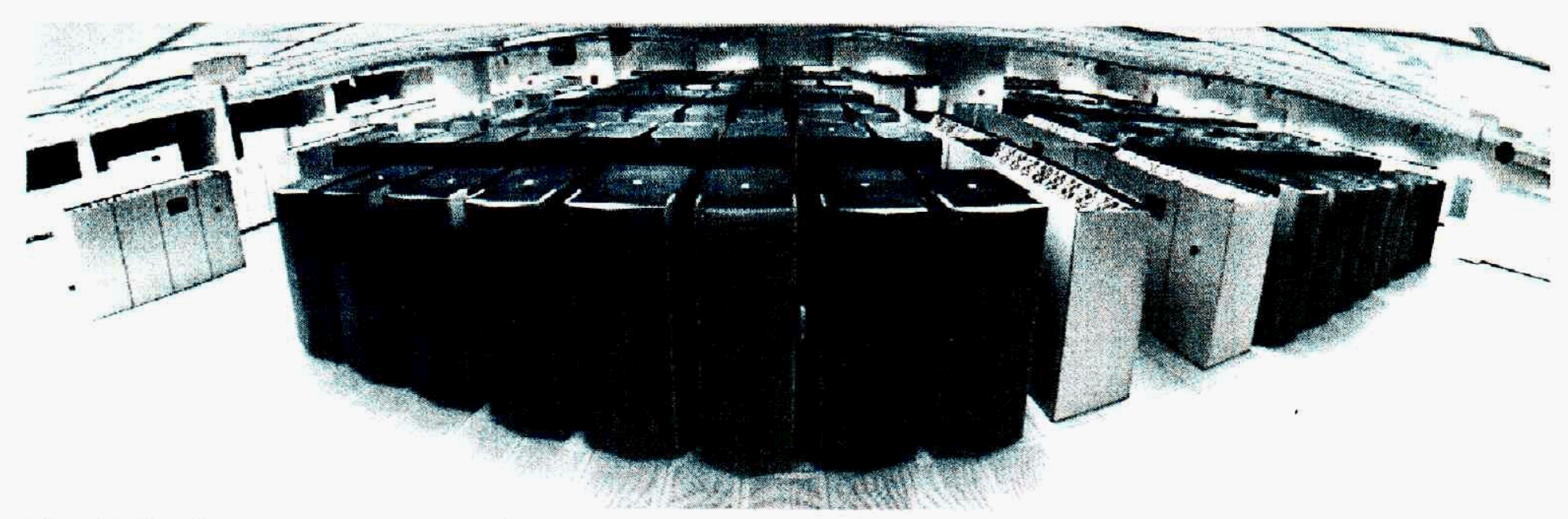

The ASCI White supercomputer, from DOE's Advanced Simulation and Computing (ASCI) Program, is capable of more than 12 trillion mathematical operations per second. It contains 8,192 microprocessors and is 40,000 times more powerful than the average personal computer. The supercomputer components occupy more than 12,000 square feet in this Computations Directorate facility. ASCI White is used in many PAT research projects. 
Fig. 23. Hubble Space Telescope data were used to support a halo location for most of the lenses seen toward the Large Magellanic Cloud. Shown here are four models tested with the "halo lensing" model, which was supported by the data.
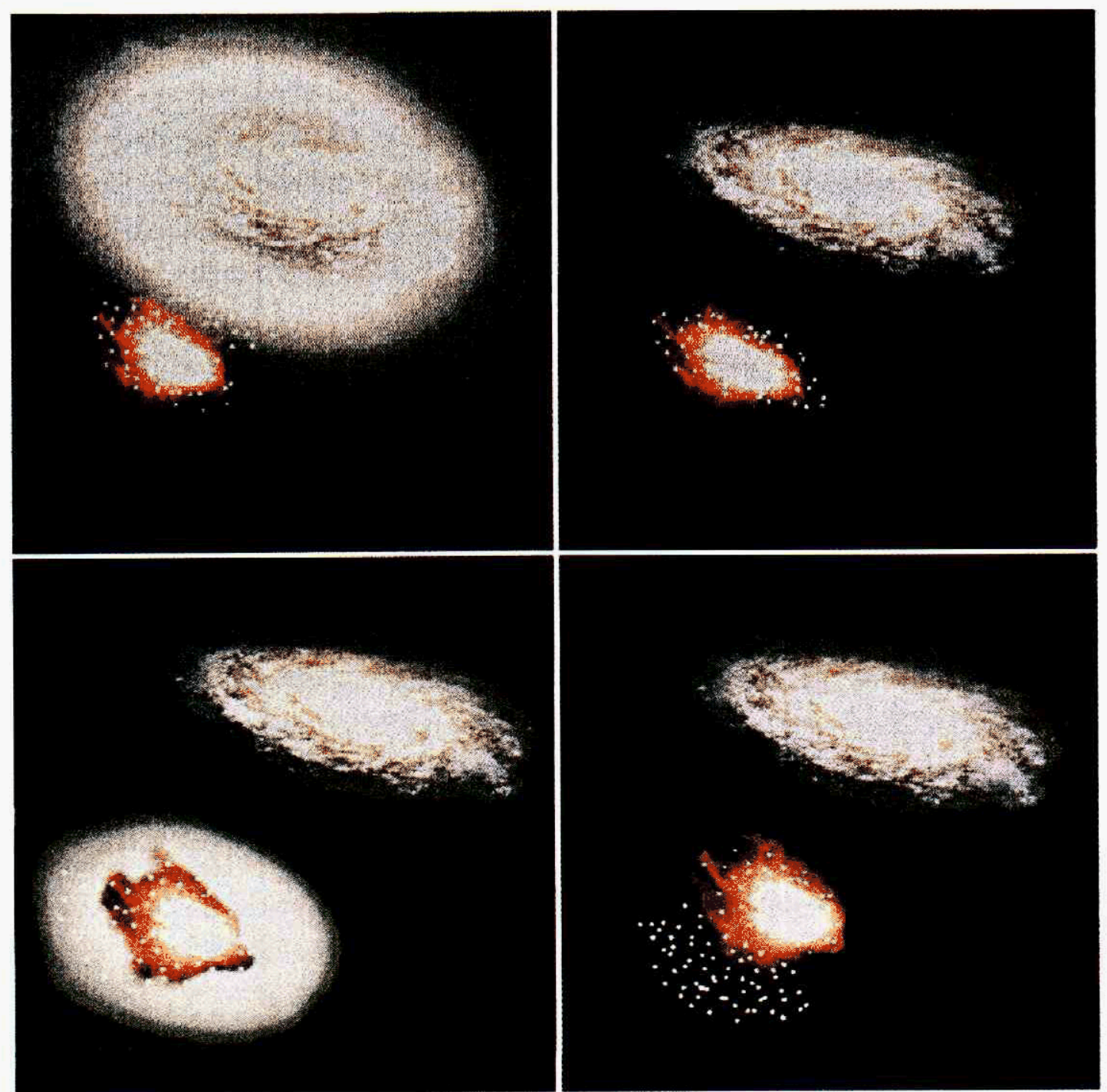

lens toward the Large Magellanic Cloud (LMC), as reported in the journal Nature; the first direct detection of a gravitational microlens; and the use of Hubble Space Telescope data to support a halo location for most of the lenses seen toward the LMC. (Fig. 23 shows four models tested with the "halo lensing" model supported by the data.) Careful analysis of the microlensing toward the bulge yielded results consonant with those predicted from far-infrared observations of the galaxy, ending a controversy caused by previous estimates of a larger amount of microlensing.

IGPP scientists are also investigating the outer regions of the solar system to better understand the size distribution of the material beyond Neptune. This material is thought to represent relatively

unprocessed material from the time of the solar system's formation. In collaboration with the Australian National University, the MACHO telescope is probing the outer solar system looking for large objects (perhaps as large as Pluto) in the region beyond the orbit of Neptune.

In collaboration with two Taiwanese institutions, another IGPP experiment, the Taiwanese American Occultation Survey (TAOS), will probe the density of small objects beyond Neptune by monitoring bright stars for occultations caused by these objects (Fig. 24). These two projects have involved significant advances in automated telescope operation and data analysis.

\section{Formation and Evolution of Structures in the Early Universe}

IGPP scientists are studying how the most massive galaxies formed in the early universe (Fig. 25). Using the two Keck telescopes and narrow-band filters, IGPP scientists have discovered gigantic emission-line halos and large-scale structures associated with a number of such galaxies when the universe was only 1 billion years old. The origin of the halos is at present not understood and may be caused by large "super winds" driven by enormous bursts of star formation, or by primordial gas that is cooling for the first 


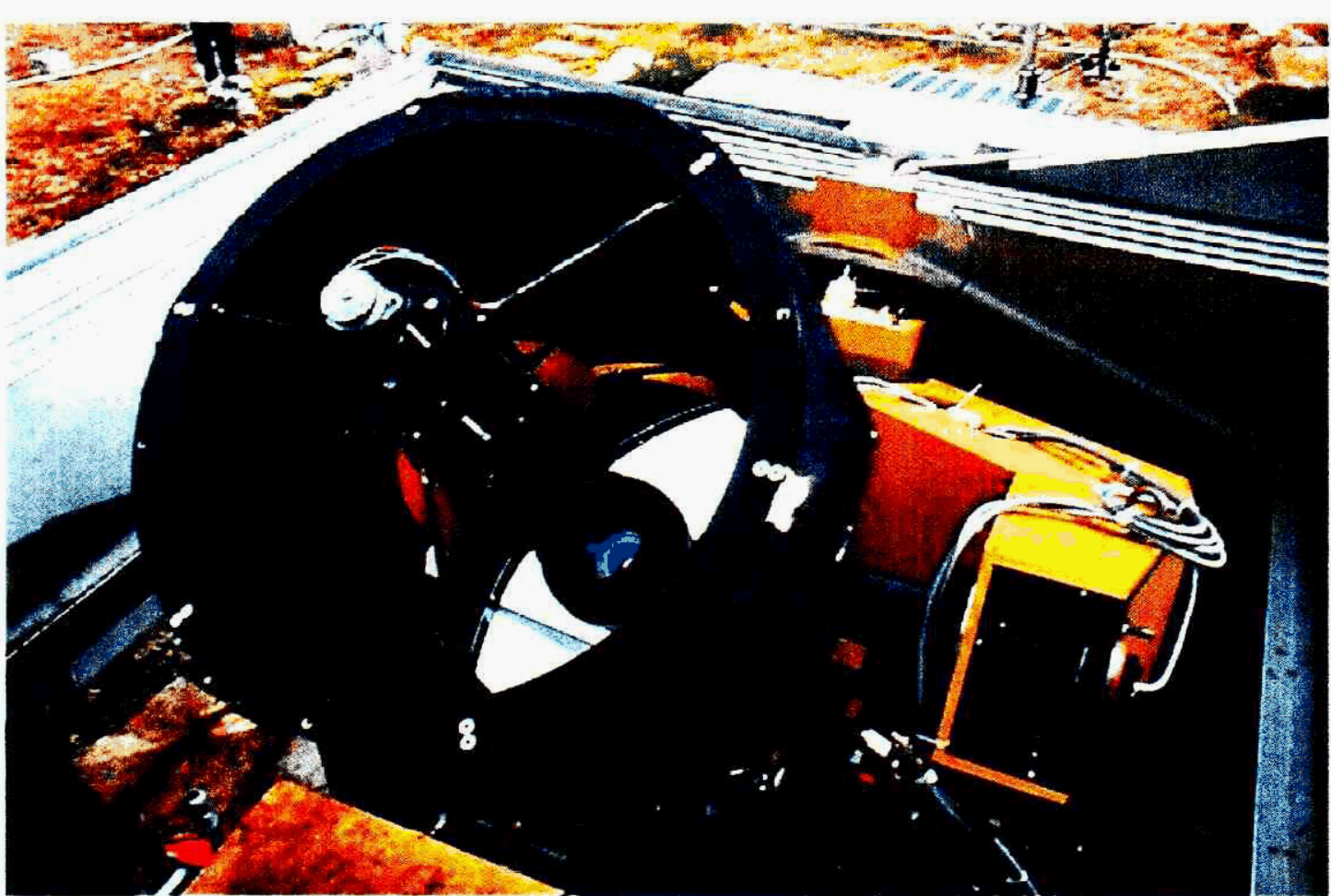

Fig. 24. The TAOS telescope is being used to probe the density of small objects beyond Neptune by monitoring bright stars for occultations caused by these objects.

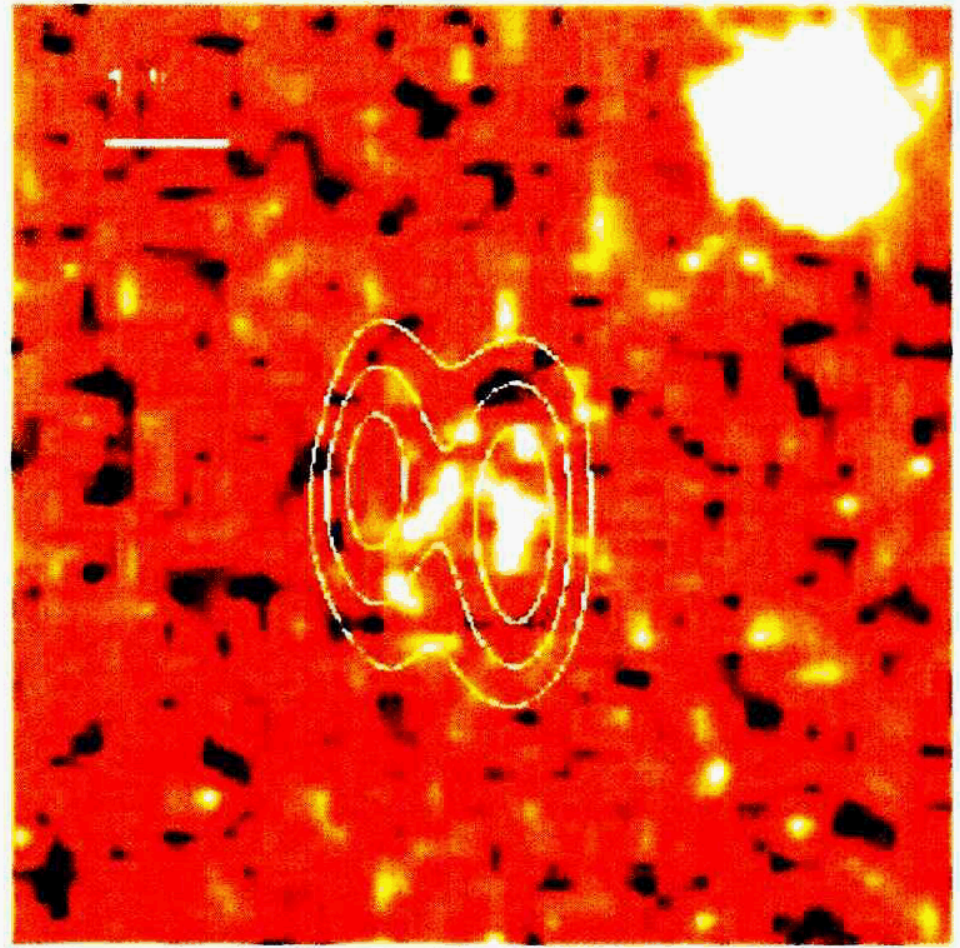

Fig. 25. IGPP scientists are studying data such as these to understand how the most massive galaxies formed in the early universe. time since the Big Bang. Further spectroscopic observations and detailed computer simulations of the hydrodynamics and radiation processes in forming galaxies will be needed to understand the astrophysical conditions in these halos.

IGPP scientists have also used the Keck adaptive optics system to investigate one of the fundamental mysteries of astronomy: the nature and origin of supermassive black holes in the centers of galaxies. It is routinely achieving resolution equal to that of the Hubble Space Telescope.

\section{Radio Surveys}

Scientists at LLNL and the University of California at Davis continue to extend the Faint Images of the Radio Sky at Twentycentimeters (FIRST) survey and extract exciting new science from it. The FIRST project is designed to produce the radio equivalent of the Palomar Observatory Sky Survey over 10,000 square degrees of the sky. It is a high-spatial-resolution survey at 21 centimeters that has discovered hundreds of new quasars, lensed quasars, and unusual radio galaxies. To date, the FIRST catalog contains 135,000 radio sources. It will be used to study quasars, extragalactic radio sources, and cosmology. FIRST scientists have won a large amount of Hubble Space Telescope time to investigate candidate lensed quasars.

\section{Djehuty Project}

IGPP is the physical home to the Djehuty Project, which is designed to develop a next-generation, fully 3-D stellar structure and evolution code running on massively parallel computers. This code and access to LLNL computers will create a center for stellar evolution study as the Djehuty Project matures. The project is already attracting outside academics who would like to collaborate on projects making use of this unique capability for the study of stars. In the future, Djehuty will become a true user facility at IGPP, providing unique access to full 3-D stellar modeling for the astrophysical community. This activity benefits from the open atmosphere at IGPP, simple access for external collaborators, and direct interaction and collaboration with the data-mining activities of the MACHO Project. 


\section{Medical Technology Program}
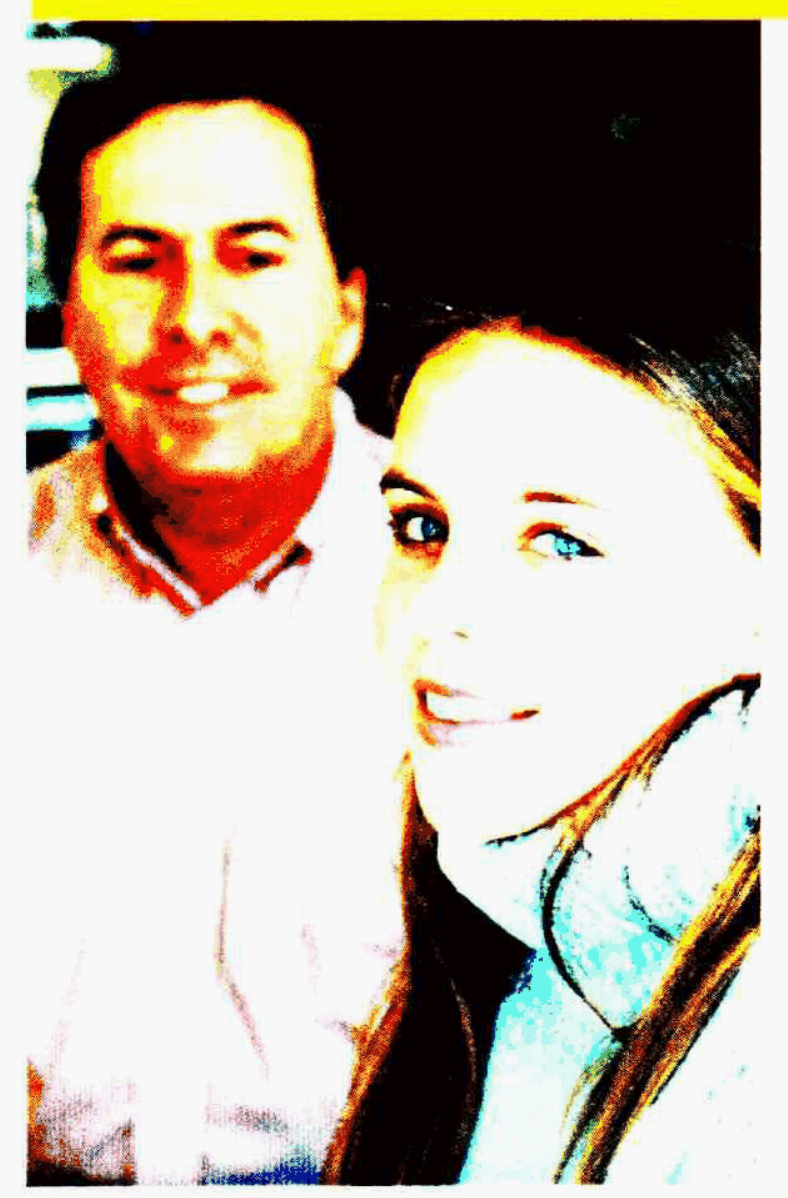

Diabetes can strike children suddenly, making them insulin dependent for life. Shown on the left is Tom Peyser, a LLNL researcher working on a PAT-led glucose monitoring technology that may help his daughter, Jenny (right). (photo credit: freelance photographer Margaret Kaye)
The Medical Technology Program (MTP) in the PAT Directorate has personnel with the skills, expertise, and capabilities that are important for advancing the biomedical science and technology needed for counter-bioterrorism systems and for developing more efficacious and less costly medical diagnostic devices and therapies. MTP provides the instrumentation necessary to study the biochemistry, physiology, and dynamic structure of living cells as part of the DOE Genomesto-Life Initiative.

MTP physical scientists are skilled at developing new instrumentation and measurement technologies that can be used for portable biowarfare-agent detection and monitoring. Included in this are core competencies in biosensors; biophotonics; mass spectrometry; micropower radar systems; and infrared, ultrasound, microwave, and x-ray imaging. These same technologies can also be used to diagnose and monitor the spread of infectious diseases, such as influenza, tuberculosis, pneumonia, cholera, and antibiotic-resistant bacteria as well as screen for the presence of various cancers and monitor their response to therapies.

MTP researchers also have considerable expertise in designing photonbased technologies to activate microtools and visualization systems for intravascular catheter systems. Additionally, experience exists in MTP for the development of micropore filters and bioreactors to make possible the development of artificial organs, such as the kidney and liver. MTP also has the computation skills and simulation codes to study photon transport within and effects upon human tissue.

\section{Microbead Immunoassay Dipstick System}

In the twenty-first century, minimally invasive and noninvasive diagnostics will form the cornerstone of clinical laboratories. In that context, the MTP is developing a tool that can be used by relatively untrained personnel (such as firefighters and paramedics) to run sophisticated, onsite diagnostics using a simple, onestep measurement. That tool is designated as the microbead immunoassay dipstick system (MIDS). (A prototype is shown in Fig. 26.) The potential applications for MIDS include point-of-care medical diagnostics (at the patient's bedside, in rural areas, or in third-world countries), portable clinical laboratories for military operations or space travel, field diagnostics for environmental sampling, and detection of biowarfare agents.

Like vehicle dipsticks that measure various fluid levels, the MIDS is designed to use optically encoded, polystyrene microbeads as templates for each assay. This approach allows a high degree of flexibility because

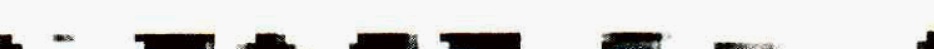

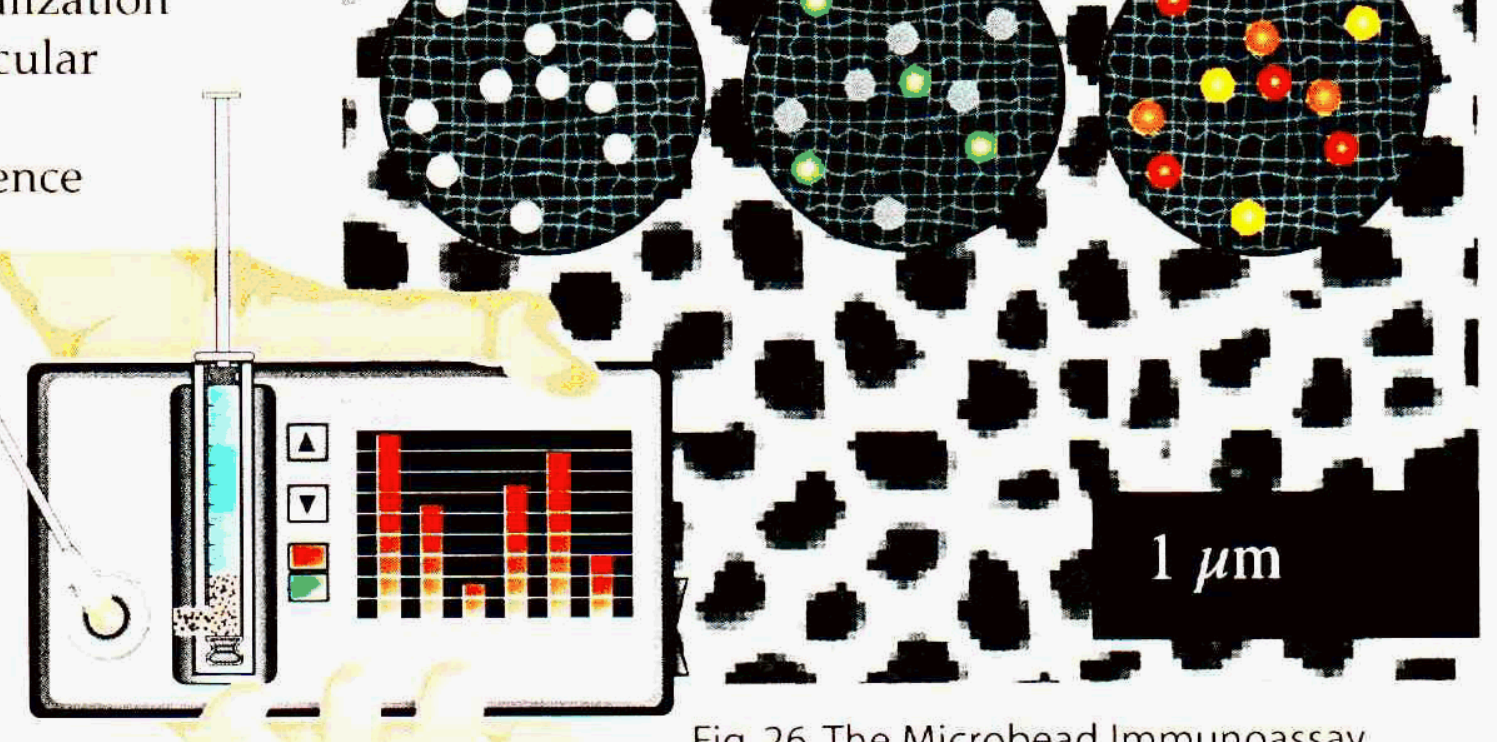

Fig. 26. The Microbead Immunoassay Dipstick System (MIDS) uses fluorescent, microbead-based assays to perform rapid, onsite diagnostics in the detection of a variety of pathogens. 
any combination of beads can be used for a given application. Incubation times are much more rapid and sensitive in the bead matrix than in conventional formats, allowing detection in minutes. Many different types of pathogens (such as viruses, bacteria, and spores) can be simultaneously analyzed in the same sample, thereby greatly simplifying sample preparation and detection protocols.

One immediate and powerful application of this device is determining how effective a particular vaccine has been in creating an immune response in individuals. In collaboration with the California Department of Health Services, LLNL's MTP researchers have developed extremely rapid, sensitive, microbeadbased assays for measuring vaccinepreventable, childhood viral infections (such as measles, chicken pox, mumps, and rubella viruses). All these assays can be simultaneously run from a single drop of blood. The use of MIDS could, for the first time, test the effectiveness of a given vaccine on individual children, eliminating in many cases the need for multiple vaccinations while ensuring $100 \%$ effectiveness.

\section{Microdot Array Sensors}

The Microdot Array Sensors (MiDAS) project focuses on developing a novel, in vivo fiber-optic sensor capable of measuring multiple biomarkers using minimally invasive delivery devices (e.g., catheters and biopsy needles). As shown in Fig. 27, these biosensors use inkjet technology to print multiple microdots on the end of a fiber-optic bundle. Each fiberoptic bundle is composed of thousands of individual fibers that are melted and drawn together in a coherent manner, allowing an image to be carried from one end of the fiber to the other. Each microdot overlaps several fibers in the bundle and contains a unique biomarker assay. Because each fiber delivers light that is spatially distinct from its neigh-

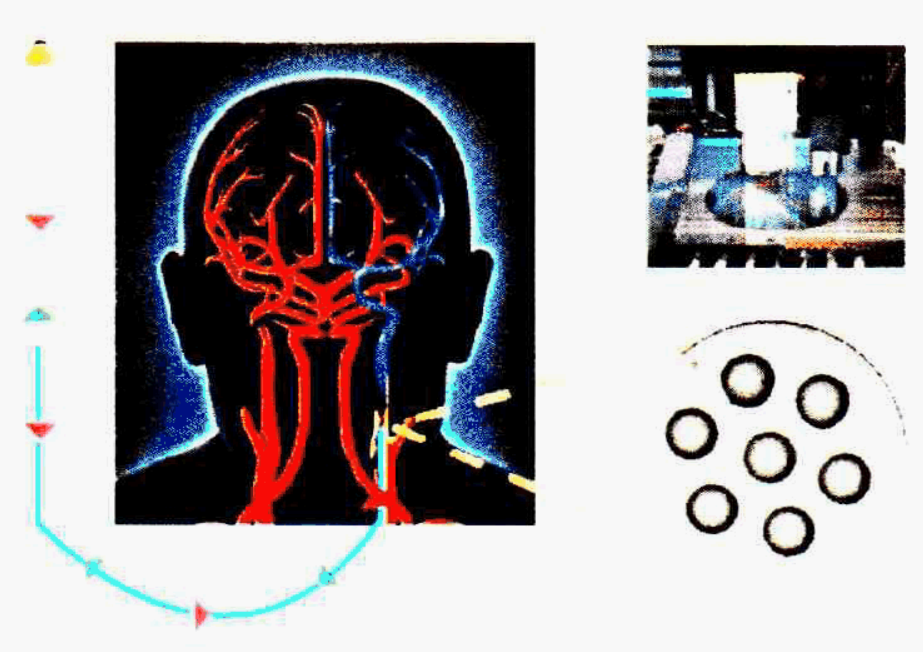

Fig. 27. MiDAS is a fiber-optic-based platform for making in vivo measurements of biomarkers, such as those associated with clot formations in strokes.

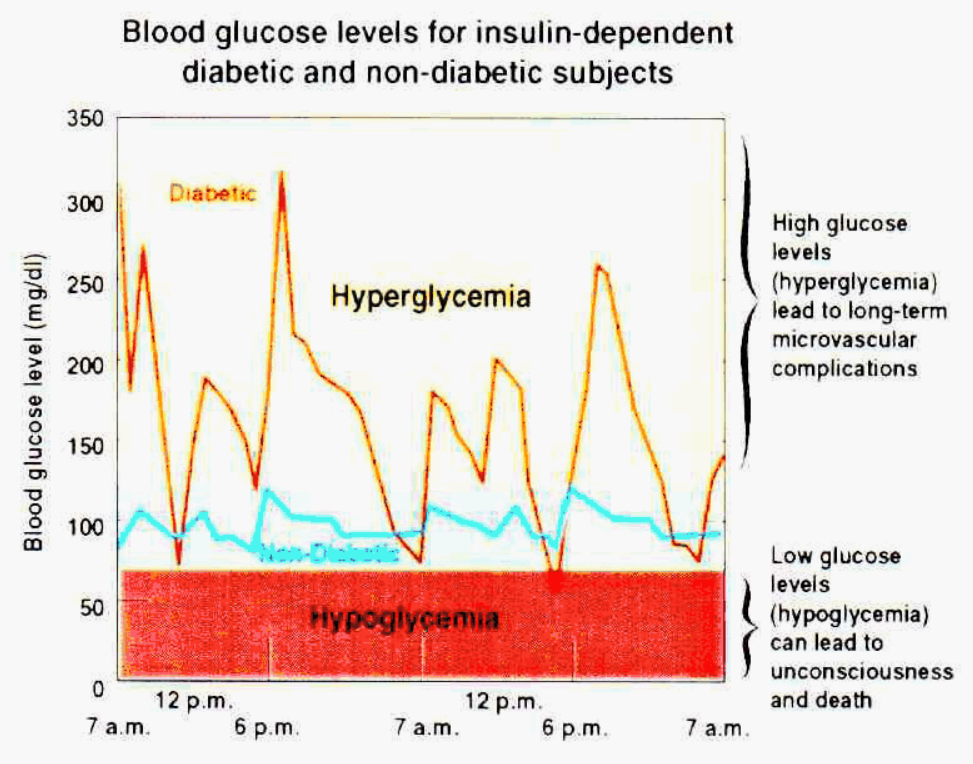

bors, it is possible to simultaneously identify each microdot and perform parallel assays by imaging the tip of the fiber bundle. One application of MiDAS is in the early detection of blood-clot formations caused by strokes.

\section{Glucose Sensor for Diabetes}

MTP's Glucose Sensor Project is developing an artificial biomechanical pancreas for use in the treatment of diabetes by combining existing glucose insulin pumps with a continuous glucose sensor. The glucose sensor will continuously monitor blood-sugar levels and communicate to the insulin pump to release insulin into the bloodstream, allowing correct insulin/blood-sugar levels to be maintained minute to minute throughout the day and night. Fig. 28 shows the variation in blood-glucose levels for nondiabetics and for a Type-I diabetic child.
Fig. 28. The graph shows measured blood-glucose levels over a 48-hour period of a nondiabetic person (blue line) and a Type-I diabetic child who is adhering to the best available treatment (red line). Over that time period, the diabetic experiences one hypoglycemic episode and many hyperglycemic episodes that extend well above the normal range. Hypoglycemic episodes can lead to coma and death. High glucose levels over many years can result in severe microvascular complications, including stroke, heart disease, kidney failure, and amputations. 
Type-I and Type-II diabetes affect 16 million people in the United States (a new case is diagnosed every 40 seconds) and more than 120 million people worldwide. The World Health Organization estimates this number will soar to 300 million by 2025. Diabetes is the single most costly disease. In 1992, total health care costs in the United States for diabetes were more than $\$ 105$ billion-approximately $15 \%$ of the national health care budget and $25 \%$ of all Medicare expenses. In 1998, diabetes was responsible for the deaths of 200,000 Americans, making it the third leading cause of death by disease in the United States.

Juvenile onset, also called Type-I or insulin-dependent diabetes, occurs when a person's pancreas produces little or no insulin, a hormone necessary to sustain life. Jenny Peyser, the teenager shown in a back cover and daughter of LLNL researcher Tom Peyser, suffers from TypeI diabetes and ultimately could be one of many who may benefit from LLNL's research on glucose sensing. Type-I diabetes is thought to be an autoimmune disease, in which the body's own system attacks and destroys the insulinproducing cells in the pancreas. Although primarily a childhood disease, adults are also susceptible.

Currently, more than 1 million Americans have Type-I diabetes. This is a disease that strikes children suddenly, makes them insulin-dependent for life, carries the constant threat of devastating complications, and shortens their lives by an average of 15 years. Conventional therapy for juvenile diabetes is to draw blood samples six or more times daily to monitor blood-glucose levels with commercial glucometers and to administer one or two injections per day of various forms of insulin. Near-normal blood-sugar levels (glycemic control) are difficult, if not impossible, to achieve with conventional therapy. Failure to achieve glycemic control inevitably leads to long- term microvascular complications, such as blindness, heart disease, stroke, kidney failure, and amputations.

Treatment is necessary for the patient to stay alive, and it requires them to extract blood samples by painful needle pricks of the fingers, followed by self-administered insulin shots. Dosages must be carefully calculated because errors can produce lifethreatening results. Normal complications of maturing are dramatically exacerbated by the demands of strict control of diet, exercise, careful testing, and self-injections. Stress, hormone changes, periods of growth, physical activity, medications, illness, infections, and fatigue also adversely affect a child's ability to control blood-sugar levels. Moreover, the social, emotional, and economic impact on families ranges from difficult at best to devastating. In fact, some families are simply unable to understand the complicated procedures necessary to administer even the minimum care required for a diabetic child.

Type-II diabetes is chiefly an adult disease in which the body cannot efficiently use the insulin it makes. The disease is often associated with obesity and poor dietary and exercise habits. With childhood obesity increasing at epidemic proportions, Type-II diabetes in children has risen at the alarming rate of $30 \%$ in the last decade.

If existing commercial insulin pumps could be combined with a continuous glucose sensor, a more physiological and fine-tuned therapy could be provided. Current research suggests that such a development would dramatically reduce morbidity and mortality from this disease.

Scientists at LLNL and its commercial partner, MiniMed, Inc., of Northridge, California, have been examining the use of dime-size implants placed a few millimeters below the surface of the skin to provide a continuous reading of a diabetic patient's glucose level. These implants contain special fluorescent-lightemitting molecules that bind specifically to glucose molecules. Upon binding and 
excitation by light at a suitable wavelength delivered through the skin, the implants produce an increased level of fluorescent light. By monitoring the portion of light that escapes back through the skin with a miniature external detector, the diabetic patient could continuously determine local glucose concentration in the body.

It should be emphasized that the present research is only in its earliest stages, but if the results continue to be promising, sensing schemes of this type could eventually be coupled to existing external or internal insulin pumps and provide nearnormal glucose control. These sensors could, in principle, provide the missing piece needed to realize an artificial biomechanical pancreas. Such a device would allow children to lead relatively normal lives and significantly reduce the risk of long-term complications.

LLNL has assembled a multidisciplinary team that includes experts in chemical synthesis, bioengineering, optoelectronics, chemical sensors, and computer modeling. The current effort at PAT is an outgrowth of a larger effort at the Laboratory to develop sensors for other defense, environmental, and biomedical applications.
The goal is to develop the prototype glucose sensor into a device that addresses the needs of diabetes patients. This device will be used in conjunction with the insulin pump from MiniMed. The goals for this project are to optimize the glucose detector, develop prototypes, and conduct early-stage clinical trials.

DOE awarded the Bright Light Award to the LLNL research team for its work developing the implantable device. The MTP team also received an Excellence in Technology Transfer Award from the Federal Laboratory Consortium for Technology Transfer for its work on the biomechanical pancreas (see the PAT Awards section).

\section{Breast Cancer Biopsy Tool: BioLuminate Smart Probe}

MTP researchers have collaborated with BioLuminate, Inc., of San Jose, California, to develop, produce, and market a revolutionary diagnostic device for early breast-cancer diagnosis. At present, 85\% of all invasive breast biopsies done each year in the United States are negative. The total cost of biopsies is $\$ 2.3$ billion annually. A multisensor probe would allow surgeons to improve breast-cancer screening and significantly reduce the number of biopsies performed.

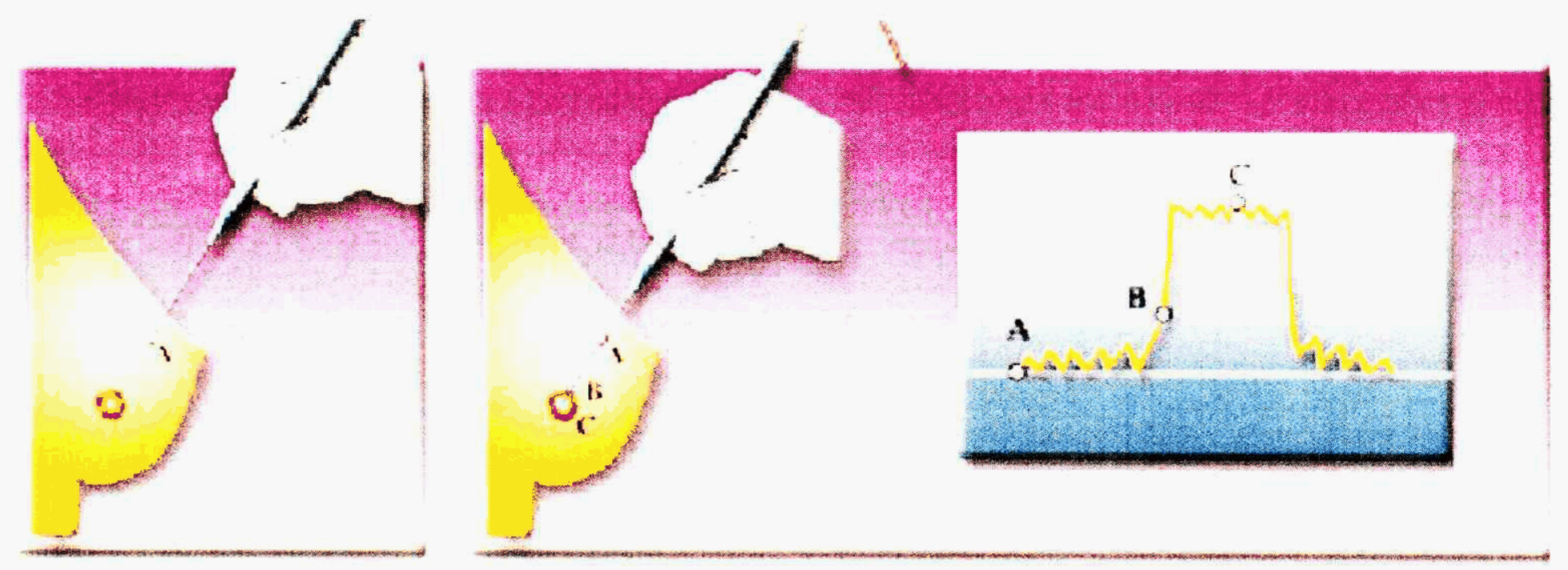

Fig. 29. The "Smart Probe" makes continuous measurements (see graph) as it moves from the surface of the breast (A) to the center of the tumor $(C)$. The graph illustrates what the analyzed signal looks like for the case of a malignant lesion. 


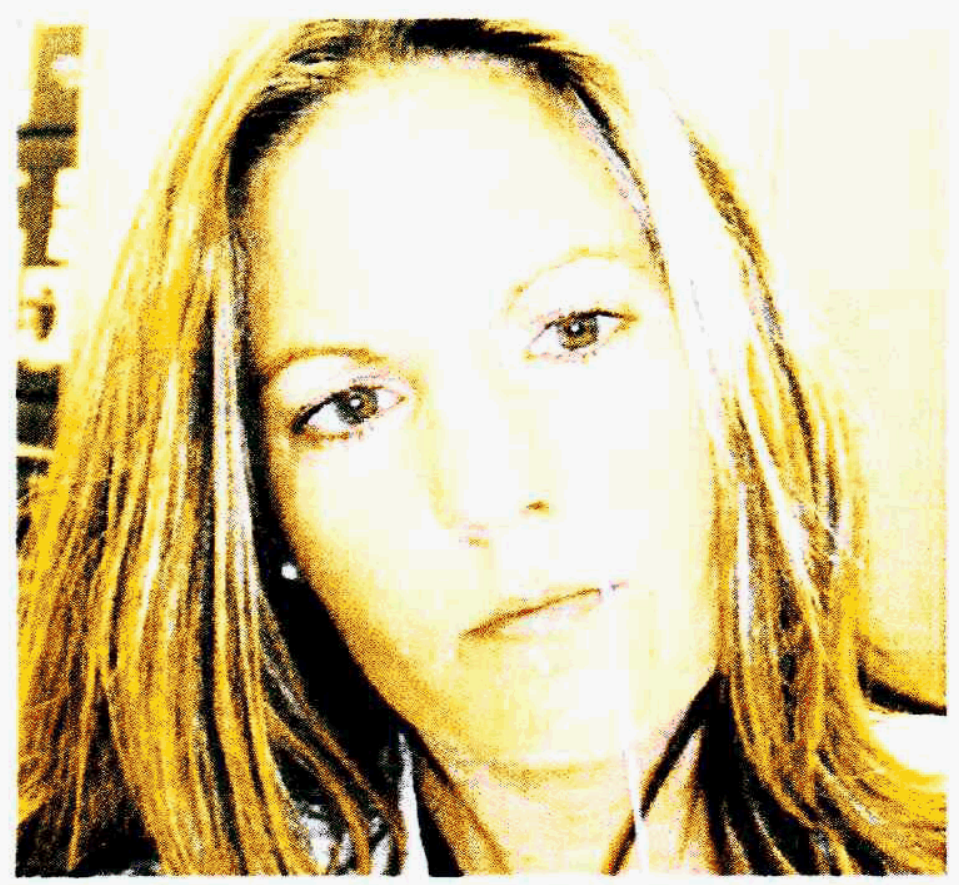

Fig. 30. Researcher Karen Lauer examines an early test version of the smart probe. The laser light output is visible at the tip of the fiber.

After a mammogram identifies a suspicious area, a physician would insert a multisensor needle - the Smart Probe (Fig. 29). This smart-needle probe, which is the size of a needle routinely used to draw blood, collects data that characterize the suspicious area.

MTP researchers have developed a sensor suite for the needle and have built more than 50 test smart-needle probes that are now being used in human clinical trials at the University of California at Davis School of Medicine. The probe, shown in Fig. 30, uses four distinct physical detection methods within each needle: optical scattering and absorption spectroscopy (OSAS), optical coherence domain reflectometry (OCDR), near-infrared (NIR) fluorescence, and a spectroscopic impedance measurement capability. Each of these diagnostic methods contributes unique

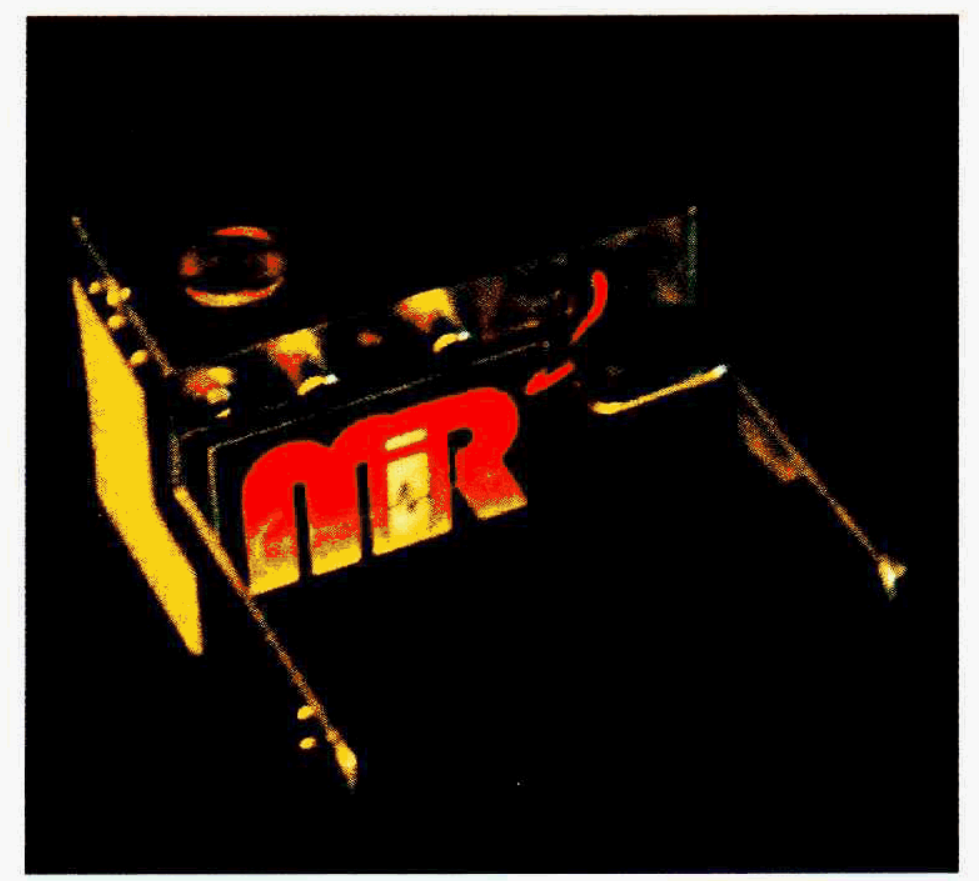

Fig. 31. The prototype MIR device has been used in feasibility studies to detect blood pooling inside the skull.

tissue information that, when combined, will give unique signatures associated with malignant or benign lesions.

\section{Handheld Radar for Detecting Intracranial Hematoma}

Blood pooling inside the skull (intracranial hematoma) can result in rapid neurologic decline and death if left untreated. A computed tomography (CT) scan, the standard method for the detection of these hematomas, requires trained technologists and large scanners and can be performed only in a hospital. A portable hematoma detector could provide a means of faster recognition and triage of these potentially lethal lesions. Such a detector would have many applications, such as in rural settings where access to a CT scanner is limited, in situations when a patient is too unstable to be brought to a scanner, and on the battlefield. 
Transmitted Pulses

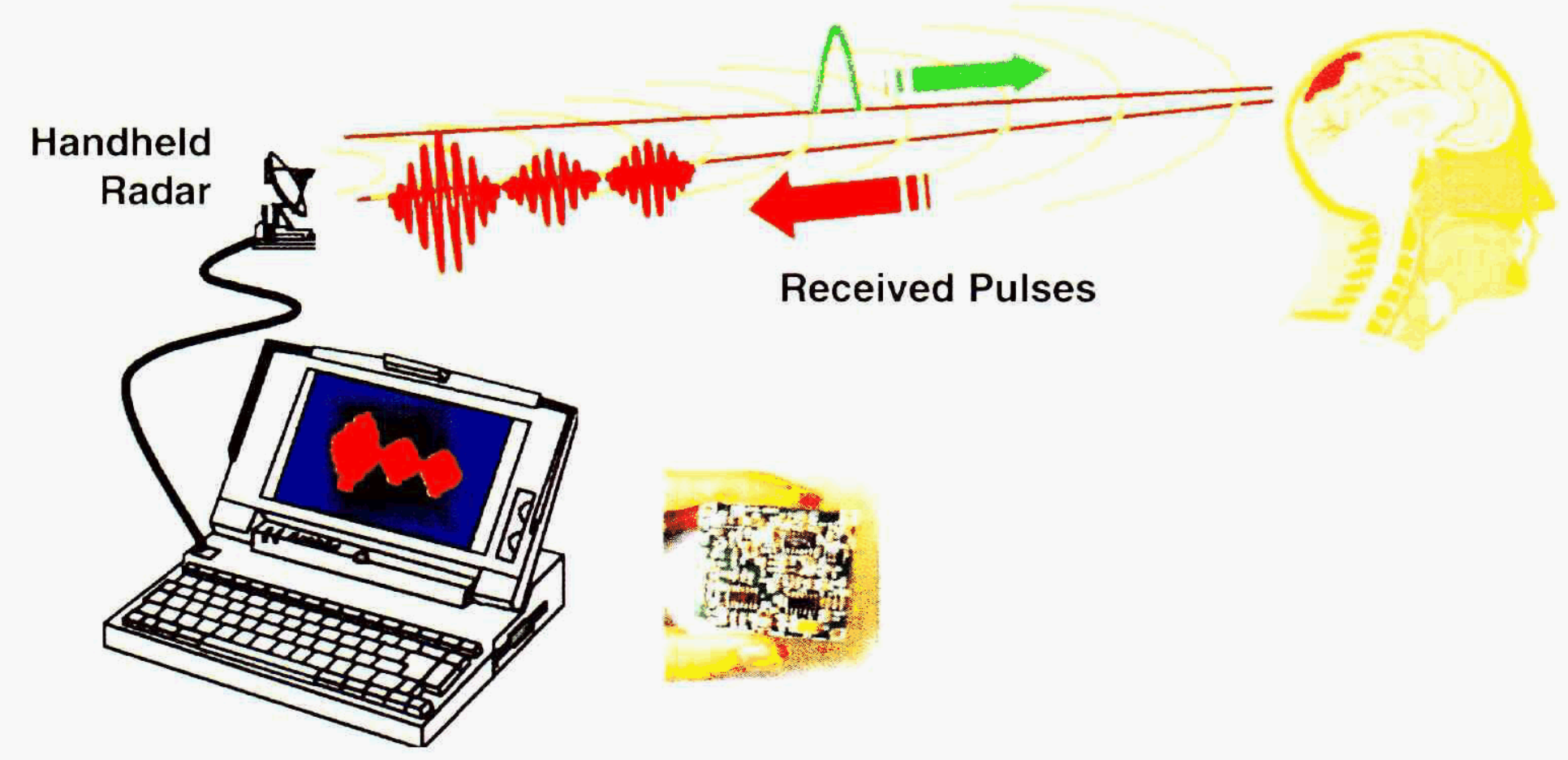

Fig. 32. Ultralow-energy radar waves interact with the suspected head trauma, generating reflections at interfaces between different tissue types. A computer displays and aids in the interpretation of the information. An early prototype of the radar transceiver board is shown in the bottom inset.

Scientists at LLNL have developed a portable, micropower-impulse-radar (MIR) device that could provide a more readily available means for detecting these lesions (Fig. 31). The technology exploits the different dielectric properties of the relevant biologic tissue by giving a reflective signal at the dielectric constant interfaces, such as at the brainhematoma interface. The MIR device is a handheld, noninvasive, ultrawideband/impulse radar that is manually scanned over the patient's head. It operates on a standard, 9-volt battery and produces a broadband radio emission that is two to three orders of magnitude lower in power than a cell phone. Operationally, the ultrawide-band, microwave-frequency impulses are reflected from the layers of tissue (scalp, dura, skull, brain, and hematoma) to a receiving antenna. Data are then collected on a laptop computer (Fig. 32).
Initial tests on phantoms (the test medium) using a cadaver swine brain indicated that this diagnostic system was feasible. In the next phase of the feasibility studies of the MIR in hematoma detection, researchers examined nonacute, nonemergency patients in a clinical setting. The individuals were examined before surgery with both the gold-standard CT scan and with LLNL's MIR device.

The results from early tests of the device are encouraging. The ability of this MIR technology to detect acute intracranial hematomas at the bedside warrants further investigation. Additional studies on human subjects will seek to improve the application-specific function of the radar and to establish sensitivity and specificity data. 


\section{Extreme Ultraviolet Lithography Pro}

Named as "Technology of the Year" by Microprocessor Report, extreme ultraviolet lithography is being developed for the manufacture of the next generation of computer chips. This test stand is located at $\mathrm{SNL} /$ California, one of PAT's partners in the Virtual National Laboratory. It will produce computer chips that can store 1,000 times more information than today's chips.
A consortium of integrated-circuit manufacturers, consisting of Advanced Micro Devices, IBM, Intel, Motorola, Infineon, and Micron Technologies, is sponsoring a Cooperative Research and Development Agreement (CRADA) at LLNL, SNL/California, and LBNL to develop Extreme Ultraviolet Lithography (EUVL). This is an eight-year, \$300-million program funded completely by the consortium members. The three national laboratories work as a single research organization, collectively referred to as the Virtual National Laboratory (VNL). The consortium members realized that the national laboratories could apply their unique expertise in soft $x$-ray optics technology to build a lithographic tool of sufficient resolution to perform lithography for all anticipated generations of silicon-based manufacturing, which is expected to last until about 2015.

\section{Printing Integrated Circuits on Semiconductor Wafers}

Lithography is the technology used to print the intricate patterns that define integrated circuits onto semiconductor wafers. Typically, a pattern on a mask is imaged with a reduction of $4: 1$ by a highly accurate camera onto a silicon wafer coated with photoresist.

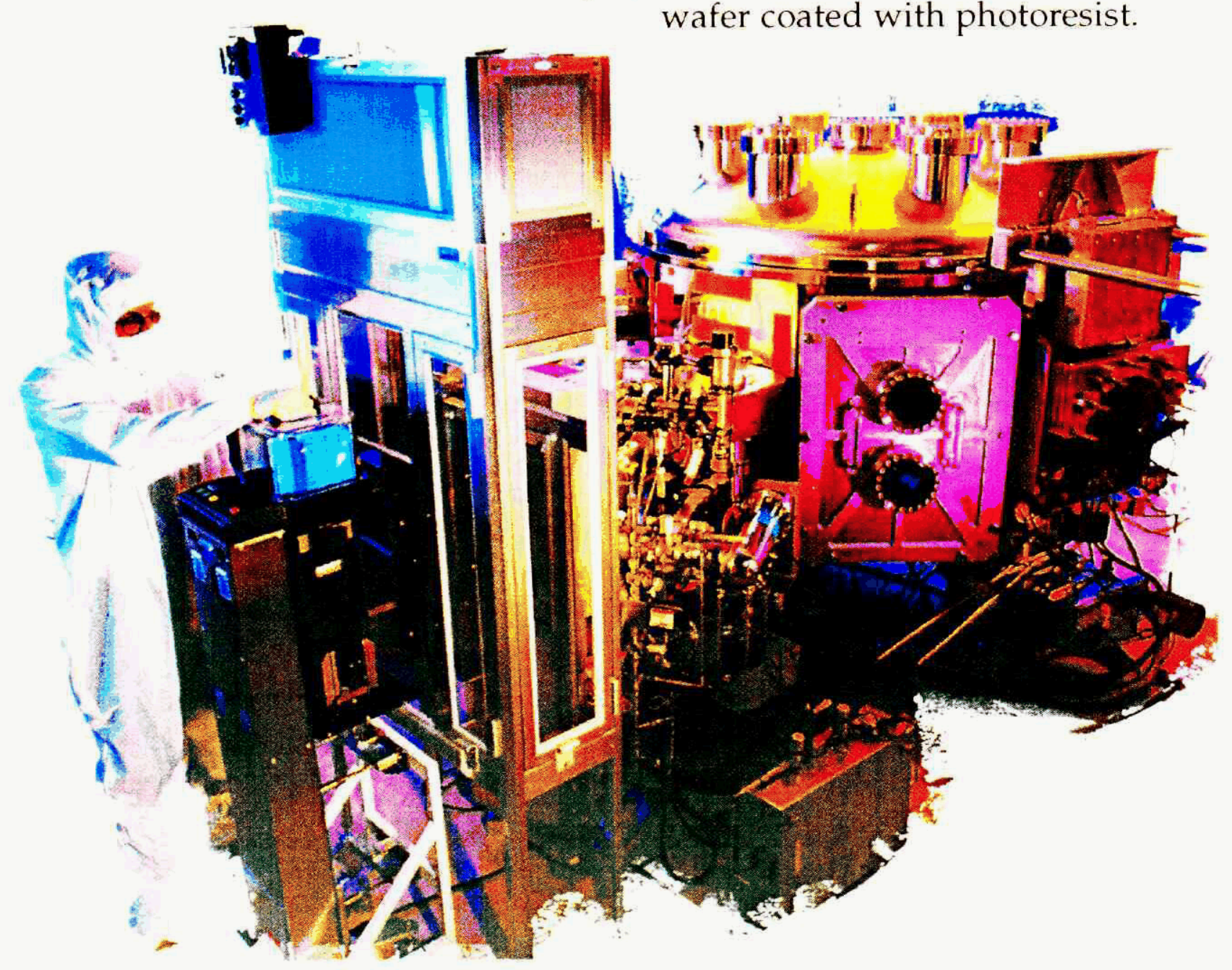

Continued improvements in optical projection lithography have enabled the printing of ever finer features - the smallest feature size decreasing by about $30 \%$ every two years. This ability, in turn, has allowed the integrated-circuit industry to produce increasingly more powerful and cost-effective semiconductor devices. On average, the number of transistors in a state-of-the-art integrated circuit has doubled every 18 months.

In order to keep pace with the demand for the printing of ever smaller features, lithography tool manufacturers have found it necessary to gradually reduce the wavelength of the light used for imaging and to design imaging systems with larger numerical apertures. Using shorter and shorter wavelengths of light has allowed the production of smaller features. Currently, the most advanced lithographic tools used in high-volume manufacturing employ deep-ultraviolet (DUV) radiation with a wavelength of 193 nanometers to print features that have line-widths as small as 100 nanometers. (A nanometer is a billionth of a meter.)

\section{Next Generation Lithographies}

After the 193-nanometer tools, the semiconductor industry plans to move to a wavelength of 157 nanometers to continue the pursuit of smaller microchip features to the 70-nanometer size. To achieve even smaller-sized features, the semiconductor industry will have to identify a new lithographic technology to propel the industry forward, eventually enabling the printing of lines as small as 30 nanometers. Potential successors to optical projection lithography are being aggressively developed. These are known as "Next Generation Lithographies" (NGLs). EUVL is the leading NGL technology; others include $x$-ray lithography, ion-beam projection lithography, and electron-beam projection lithography. 
Fig. 33 shows the basic elements of an EUVL system. A pulsed laser beam trained on a jet of xenon gas creates a plasma (not shown) that emits 13nanometer radiation, which is focused onto a mask. The mask reflects its circuit pattern onto a series of curved mirrors that reduce the size of the image and focus the image onto the wafer. The thicknesses of the 80 alternating layers of silicon and molybdenum that make up the mirrors must be accurately controlled so that both the thickness of each layer and the total thickness over the surface deviate by less than one atom. The entire circuit-printing process must be done in a vacuum because air absorbs radiation at this wavelength. Furthermore, the mask distorts the image if it contains more than a handful of defects measuring as small as 5 nanometers (about 20,000 times narrower than the width of a human hair).

In many respects, EUVL may be viewed as a natural extension of optical projection lithography because it uses short-wavelength radiation (light) to carry out projection imaging. In spite of this similarity, there are major differences between the two technologies. Most of these differences occur because the properties of materials in the EUV portion of the electromagnetic spectrum are very different from those in the visible- and ultravioletwavelength ranges.

Foremost among those differences is the fact that EUV radiation is strongly absorbed in virtually all materials, even gases. Because of this absorption, EUV imaging must be carried out in a nearvacuum. Absorption also rules out the use of refractive-optical elements, such as lenses and transmission masks. Thus, EUVL imaging systems are entirely reflective.

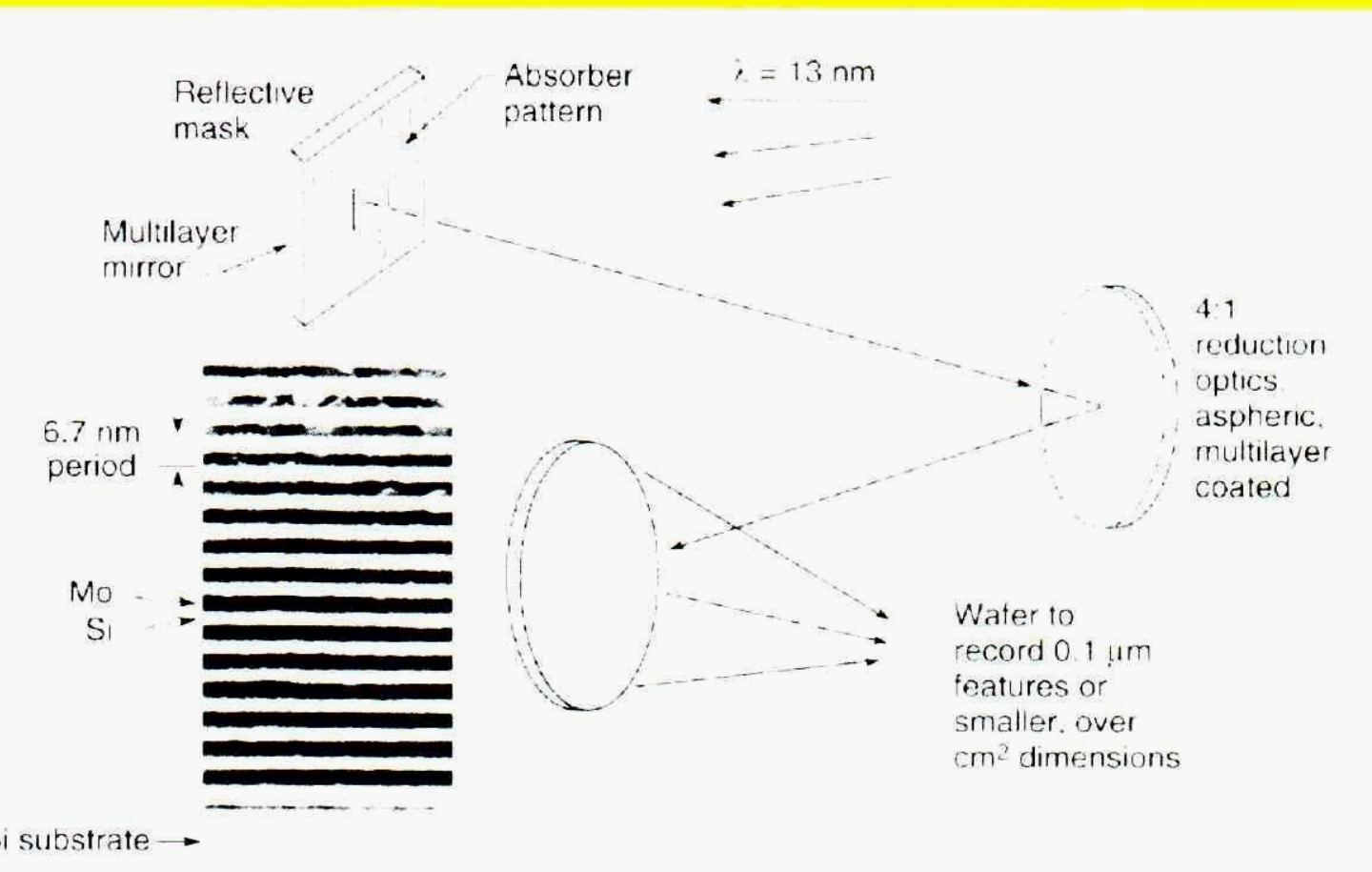

Ironically, the EUV reflectivity of individual materials at near-normal incidence is very low. In order to achieve reasonable reflectivities near normal incidence, surfaces must be coated with multilayer, thin-film coatings known as distributed Bragg reflectors. The best of these function in the region between 11 and 14 nanometers. Additionally, EUVL requires new resists and processing techniques compared to standard optical or projector lithography. Finally, because EUVL utilizes short-wavelength radiation for imaging, the mirrors that make up the camera must exhibit an unprecedented degree of perfection in surface figure and surface finish in order to achieve diffraction-limited imaging. Fabrication of mirrors exhibiting such perfection will require new, more accurate polishing and metrology techniques.

Ultimately, this system will enable a microchip to be manufactured with etched circuit lines smaller than 100 nanometers in width, extendable to below 30 nanometers. The resulting microprocessors will be a hundred times more powerful than those made today. Memory chips will be able to store a thousand times more information than at present.
Fig. 34. Basic layout of an EUVL optical system. All the optical elements are reflective and coated with multilayers, such as the molybdenum-silicon mirror example shown in the blowup insert on the left. Each surface has a reflectivity of 13.4 nanometers. The system must be operated in a vacuum. about $70 \%$ at 


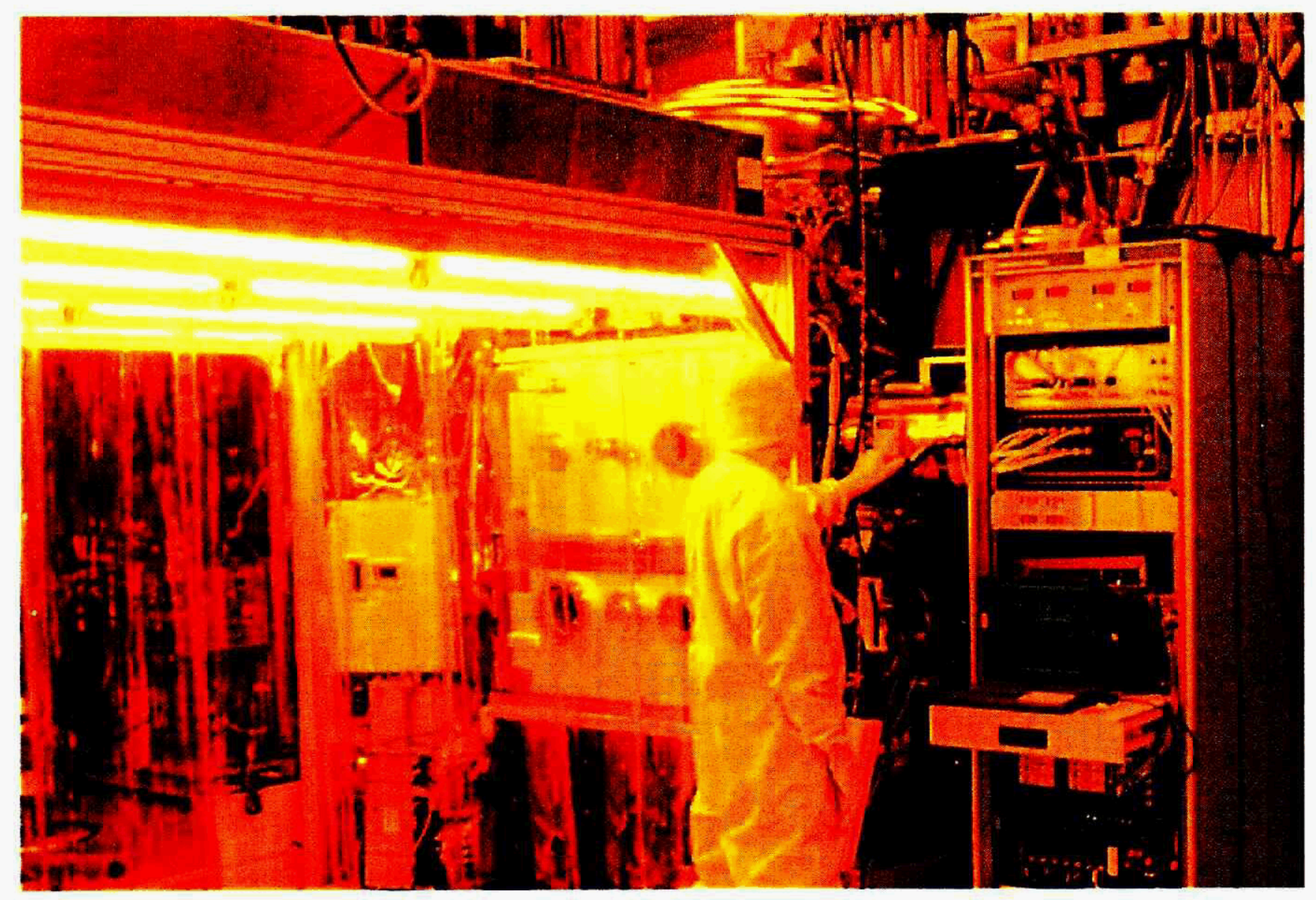

Fig. 35. The

Engineering Test Stand was built by SNL/California, LLNL, and LBNL under the sponsorship of a consortium led by Intel. The tool can print a 25-millimeter-square image with 70-nanometer resolution and uses the soft $x$-ray wavelength of 13.4 nanometers.

\section{LLNL Contributions in the EUVL Program}

Each of the national laboratories participating in the CRADA brings unique contributions to this effort. LLNL is the lead laboratory for optical design, optical fabrication, metrology, multilayer coating, and mask fabrication. The accomplishments in several of these LLNL-led areas are highlighted here.

The EUV Optics Group at LLNL, in conjunction with partners at LBNL and SNL/California, have recently delivered two projection imaging systems for use on an alpha-class EUV lithographic exposure tool (Fig. 34). Each system has taken its turn as the most accurate large-field imaging system ever built with wavefront errors of about 1 -nanometer rms. The printing of 80-nanometer line features using this optical system has been demonstrated (Fig. 35). Meeting this challenge has required advances in the state of the art in several disciplines that contribute to the overall optical system.

To realize the optical design, mirror substrates must be polished with outstandingly low levels of errors. In addition to being highly reflective, the mirrors must have surface coatings that are essentially perfectly uniform. For the prototype system, the mirror surfaces had to have geometric-shape (figure) errors less than 0.25-nanometers $\mathrm{rms}$ and a surface finish of 0.1- to 0.2-nanometers rms over clear aperture dimensions of 50-150 millimeters. L.LNL developed a collaborative relationship with ind ustrial optics suppliers. Together, they have improved the state of the art in simultaneously meeting figure and finish by factors of two to three times that of previous best efforts. It is important to underscore the great difficulty in achieving this goal with the aspheric shapes that are required for EUVL imaging systems.

Polishing the substrates to such an incredible accuracy is possible only if there is metrology available to both validate the specifications and provide feedback for the finishing process. A new style of interferometry has been developed to provide the substrate manufacturer with a detailed map of shape errors. With such a map, the errors are addressed in subsequent polishing iterations. This absolute interferometer instrument employs accurate spherical waves that are produced by the diffraction of light from pinholes that are used as an absolute reference for making a direct assertion of accuracy for the aspheric surfaces. The VNL Team at LBNL performs angle-resolved scattering measurements that give a functional validation to surface roughness measurements that are obtained using commercial instruments. A successful partnership between the national laboratories and industry offers the best chances for achieving the continued improvement in figure and finish that is necessary for construction of the EUV production tools.

\section{Multilayer Coatings}

Perhaps the single most enabling technology in constructing an EUV imaging system is the deposition of multilayer coatings. These coatings provide a suffi- 
ciently high reflectance to allow the use of near-normal incidence optics. Before the existence of these multilayers, researchers employed either grazing incidence optics or zone plates-neither of which offered the degrees of freedom necessary for controlling aberrations to the levels required for EUVL. For several years, LLNL's Multilayer Coatings Group has been the leading organization in developing coatings and in applying them for programmatic applications. The key specifications that are addressed include high reflectance at the operational wavelength (13.4 nanometers), coating-thickness control so that the accurate substrate shapes are not deformed, and tight wavelength control so that the bandpass of the coatings is well matched among all the surfaces. LLNL's Multilayer Coatings Group met each of these challenges in the various categories for the prototype system and is close to satisfying the final requirements needed for future lithographic production tools.

Modeling the optical system is critical in determining the tool's lithographic performance. LLNL's Optics Group has developed models for calculating 2-D aerial images, using realistic models for the partially coherent illumination. These models also allow the input of the system wavefront errors that are determined during alignment, and the power spectral density data that describe flare.

Furthermore, mask patterns, such as elbows or stars, can be used in the models to determine the intensity profile at the image plane and to calculate contrast, critical dimension (CD) variation, linearity, image placement errors, and throughfocus behavior. This simulation capability has proven especially important in analyzing printed patterns from the prototype exposure tool and determining if any alignment adjustment is required, or if the printed images show expected trends.

\section{A Successful Partnership}

The development and the construction of a sophisticated optical system, such as that used for EUVL, requires a systemslevel understanding of each component technology and how it relates to the functional specifications. The accomplishments attained for the EUVL program have demonstrated a successful partnership - not only among diverse technical groups at LLNL but also with other national laboratories that possess key technical expertise, among the industrial suppliers that strive to translate feasibility demonstrations into commercial products, and the semiconductor companies that offer insight into the functional specifications. This partnership and its achievements were recognized in April 2001 at the EUVL Milestone Celebration (Fig. 36).

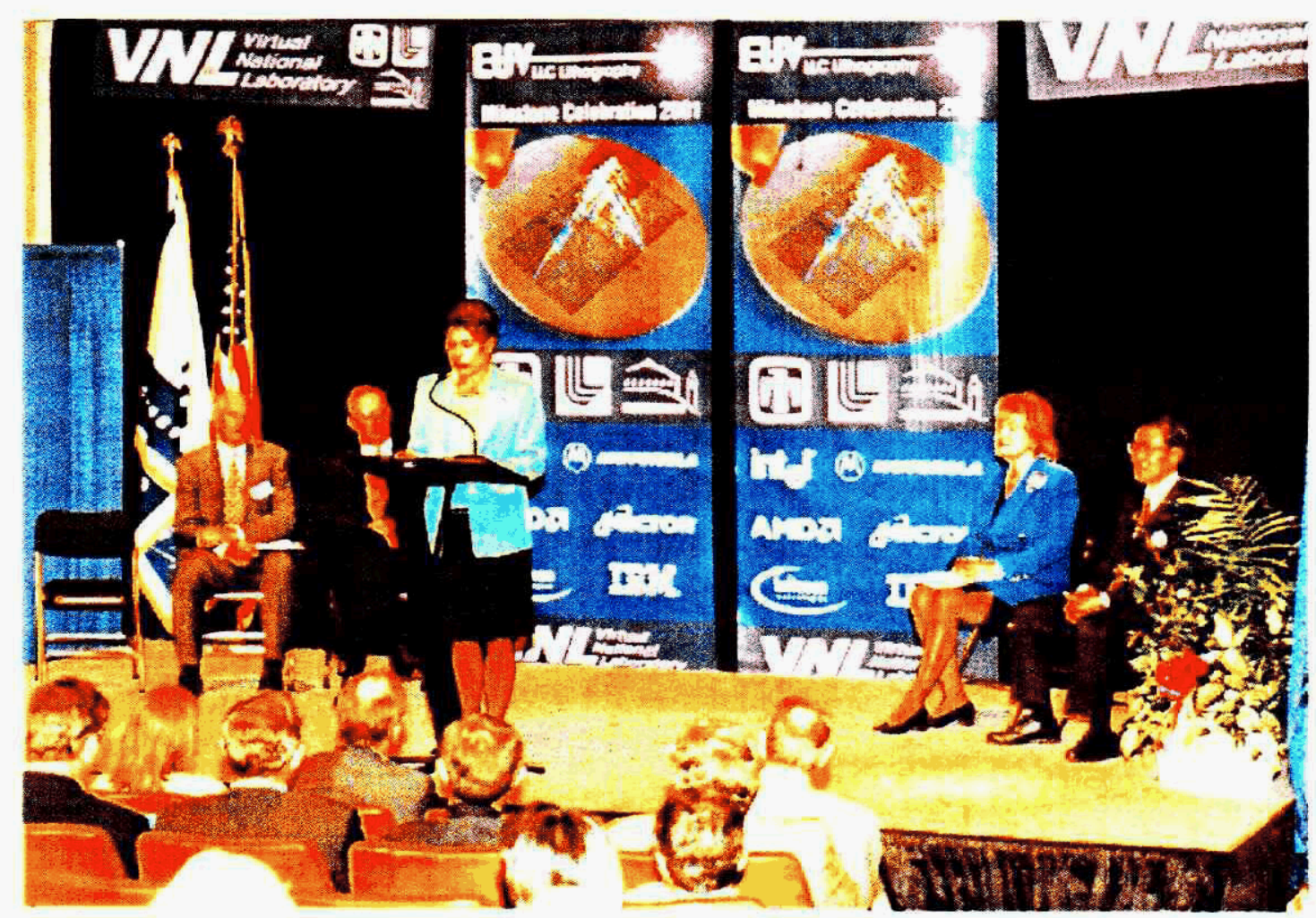

Fig. 36. On April 11, 2001, more than 200 people attended the EUVL Milestone Celebration to recognize the first operation of the Extreme Ultraviolet Lithography tool built by three national laboratories. Dignitaries shown at the dais from the left are Dr. Craig Barrett, Chief Executive Officer, Intel Corporation; General John Gordon, Undersecretary for Nuclear Energy; and National Nuclear Security Administrator Dr. Miriam John, Vice President, Sandia National Laboratories, California; the Honorable Ellen Tauscher, U.S. House of Representatives (Democrat); and Sunlin Chou, Senior Vice President and General Manager for the Technology and Manufacturing Group, Intel Corporation. 


\section{Imaging and Advanced Detectors Di}

During 2001, the PAT Directorate consolidated its imaging, detection, and optical sciences work into a new effort, Imaging and Advanced Detectors Division (known as I Division). Activities in the newly formed I Division include adaptive optics, image processing and visualization, optics and metrology, multilayer technology, advanced detectors, photonics, highpower laser systems, and advanced intercept technology. (The optics, metrology, and the multilayer technology activities supporting the EUVL Program are reported in the previous section.) Highlights of I Division activities in 2001 are described below.

\section{Adaptive Optics}

The activities of the Adaptive Optics (AO) Group in I Division included projects with application to high-power lasers, laser communications, remote sensing, astronomy, and human vision. See Fig. 37 for a summary portrayal.

In the areas of optical communications and remote sensing, a collaborative team led by LLNL was selected for funding support (approximately $\$ 9.5$ million) as the Coherent Communications, Imaging and Targeting Program through the U.S. Department of Defense's Defense Advanced Research Projects Agency (DARPA). This team is developing powerful, new capabilities for secure, freespace communication links (at rates of multigigabits per second) and aberrationfree, 3-D imaging and targeting at very long ranges (more than 1,000 kilometers). Innovative concepts and integration of micro-electromechanical-systems (MEMS) spatial-light modulators (SLMs), which provide a quantum leap in wavefront control, advanced photonics components, and high-speed electronics will provide affordable and high-value systems for use well into the twenty-first century.

Phase I of the two-phase program will be executed by a team consisting of LLNL, four universities (Boston University, Stanford University, University of California at Berkeley, and Georgia Institute of Technology), several MEMS/photonics companies (Boston Micromachines, Lucent, and MicroAssembly Technologies), and major U.S. aerospace companies (Ball Aerospace, Boeing, Harris, HRL Laboratories, Lockheed Martin, Northrop Grumman, Raytheon, TRW, and Aerospace Corporation). Phase I funding is for a two-year period that began in May 2001. Successful quarterly reviews for this project were held in October 2001 and January 2002.

Another project in the area of optical communications is a collaborative effort with LLNL's Nonproliferation, Arms Control \& International Security (NAI) Directorate on the Secure Air-optic Transport and Routing Network (SATRN) Strategic Initiative. For this project, an adaptive optics system is being developed based on MEMS deformable-mirror technology to compensate for atmospheric degradation of free-space laser communications system performance.

In the area of high-power laser systems, in collaboration with the Laser Science \& Technology (LS\&T) Program, I Division is developing a solid-state, heat-capacity laser. Supported by a contract with the U.S. Army, this project is utilizing an adaptive optics system inside an unstable resonator cavity in order to compensate
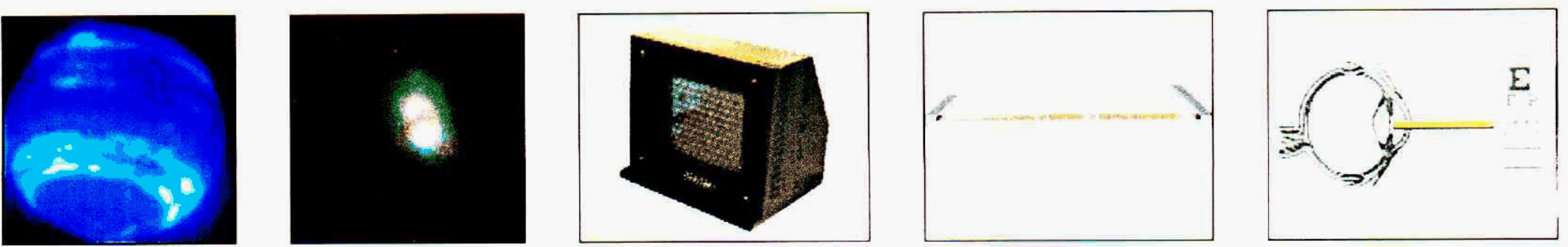


\section{vision (I Division)}

for optical aberrations in the gain media. These aberrations lead to degradations in beam quality that limit the ability to bring the beam to a sharp focus on a target.

In 2001, the adaptive optics system designed for a 10-kilowatt laser system was implemented and tested; and its functionality was demonstrated. A successful comprehensive external review of the program was held in November 2001. Further refinements of the adaptive optics system are planned in 2002 to optimize its performance. Another project in this technical area involves collaboration with the LS\&T Program to develop programmable phase plates, based on liquid-crystal SLM technology, to provide a beam-shaping capability for a highpower laser drilling system. The base beam-shaping system has been designed and tested.

In the area of astronomy, the AO Group has been involved in the development and operation of the laser guide star adaptive optics system for the 3-meter telescope at the University of California's Lick Observatory. Members of the $\mathrm{AO}$ Group aided astronomers who are using this unique system to produce high-resolution images of galaxies, protostellar regions, and planets in the solar system. A laser guide star system was subsequently installed in the 10-meter telescope at the Keck Observatory to enhance the existing adaptive optics system (see front cover photo). The first tests projecting the Keck laser to the sodium mesophere were conducted successfully in January 2002.

The AO Group has also been intimately involved with two other large-telescope adaptive optics systems now in their design phases: the Gemini national 8-meter telescope and the 30-meter California Extremely Large Telescope (CELT). The Gemini system, which will be the first to use multiple laser guide stars to correct over a wide field, is now in the final stages of design. The CELT is at the conceptual design and analysis stage. Members of the AO Group contributed substantially to a chapter on adaptive optics in the final report on the CELT conceptual design and analysis.

\section{Vision Correction with Adaptive Optics}

In the area of human vision, the AO Group began a new collaborative project with the Department of Ophthalmology at the University of California at Davis. With support from Laboratory Directed Research and Development (LDRD) funding, the project will assess the limits of human visual acuity by using liquid-crystal SLM wavefront corrector technology.

With funding support from the National Science Foundation's Center for Adaptive Optics, the AO Group also collaborated with the University of Rochester to test the use of MEMS deformable mirrors to correct aberrations of the eye. Based on these activities, a collaborative team led by LLNL has been selected for support (at the level of approximately $\$ 2.7$ million) through the DOE Biomedical Engineering Program. Over a two-year period, this team will develop and test clinical ophthalmic instruments that use MEMS adaptive optics in hopes of revolutionizing the diagnosis and treatment of the diseases that cause blindness, and the techniques for vision correction in the general population. Diseases addressed by these new instruments and the newly enabled clinical
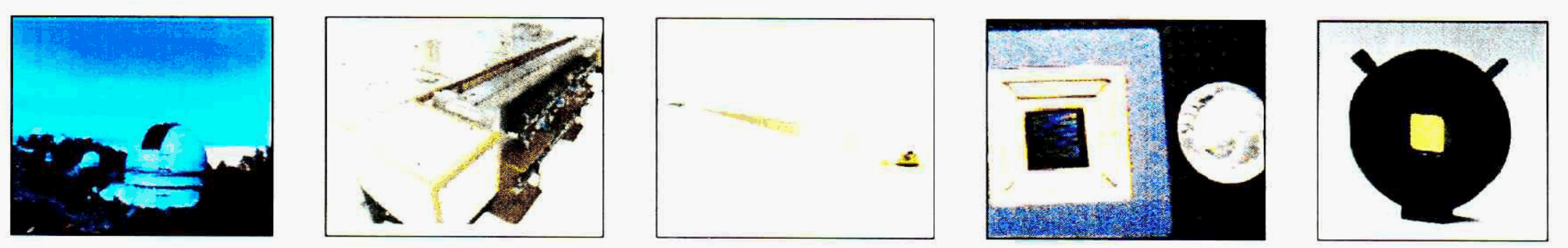

Fig. 37. The activities of the PAT Adaptive Optics Group included ongoing projects on astronomical and high-power laser systems using conventional deformablemirror technology as well as new projects in laser communications, remote sensing and imaging, and vision science using new micro-electromechanical (MEMS) and liquidcrystal spatial-light modulator (SLM) technology. 


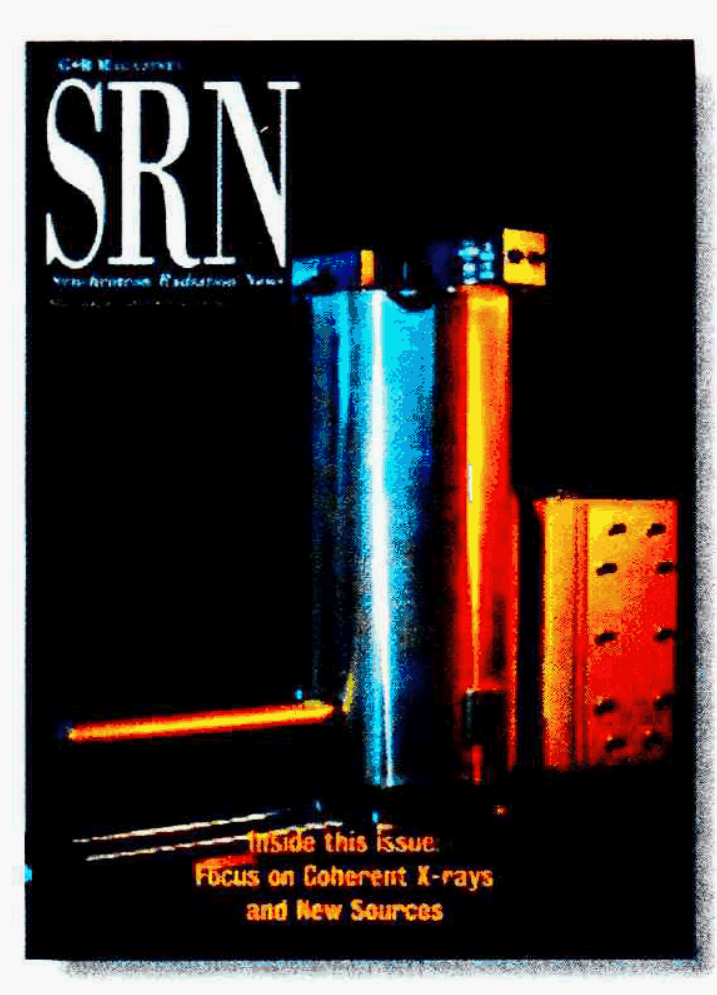

Fig. 38. PAT's work in superconducting tunnel junction $x$-ray detectors was featured in the March/April 2001 issue of Synchrotron Radiation News. protocols include the main causes of blindness in the United States: macular degeneration, glaucoma, diabetic retinopathy, and retinitis pigmentosa. The project team includes multiple DOE national laboratories (LLNL and SNL) and multiple medical research centers (University of California campuses at Davis and Berkeley, University of Rochester, and Johns Hopkins University), along with the Army Aeromedical Research Laboratory and Bausch \& Lomb Corporation. Additional support by Bausch \& Lomb and the Center for Adaptive Optics will allow the evaluation of the clinical utility of this instrumentation for vision correction, and determination of the suitability of this instrumentation for commercialization.

\section{Advanced Detectors}

The Advanced Detector (AD) Group in I Division is developing high-resolution and high-sensitivity detectors for use from the near-infrared spectral region to gamma-ray wavelengths. In the nearinfrared wavelength, an array of detectors will form a high-sensitivity camera with near-zero readout noise. Combined with a large telescope and an adaptive optics system, this near-infrared camera will be capable of directly measuring light from planets orbiting nearby stars. At x-ray energies, the detectors combine high-spectral resolution with high efficiency over a wide energy band. Such detectors are ideal for both astronomical measurements and synchrotron-based spectroscopy of biological samples. At gamma-ray energies, the detectors can measure the ratio of nuclear isotopes with high precision for nonproliferation and arms control applications.

Several types of detectors are being developed-all of which operate at temperatures near 0.1 Kelvin-approaching absolute zero at zero Kelvin. When the detectors are cooled to these low temperatures, the energy of the individual photons becomes a significant fraction of the energy of the entire detector. In a microcalorimeter, each photon warms up the detector; and this pulse in temperature is measured with high precision. In a superconducting tunnel junction, each photon breaks from thousands to millions of superconducting Cooper pairs, which are then counted.

Last year, the AD Group optimized the fabrication of molybdenum and copper transition-edge sensor microcalorimeters and developed a process to consistently produce hundreds of high-quality devices simultaneously. The current focus of the AD Group is on optimizing the operation of the devices. In addition to the standard, single-pixel operation, the group began a major thrust toward multiplexing arrays of detectors with a single Superconducting Quantum Interference Device (SQUID) amplifier. A single detector was successfully operated with a highfrequency, alternating-current bias; and the demodulated signal produced gamma-ray spectra with a resolving power of 1000 . The next step is to run several detectors at different bias frequencies, sum the signals together with one amplifier, and separate the different channels from the amplifier output.

The AD Group operated superconducting tunnel junction detectors in a 3-by-3 array. This array can potentially achieve a total counting rate above 100 kilohertz. Each detector in the array is evaluated with its own amplifier. The array was operated at the Advanced Light Source X-ray synchrotron at LBNL to identify transition metals in proteins.

Transition-metal complexes are key elements in many biologically important processes that are catalyzed by proteins (enzymes). Photosynthesis is a prime example. The changes that occur in electronic structure throughout a catalytic cycle are the subject of much research aimed at understanding the mechanisms of these processes. L-edge x-ray spectroscopy is a powerful tool that can be used to study such mechanisms. The concentrations of the transition-metal complexes in enzymes are, however, typically low - around 100 parts per millionso that the metal signal rides on top of a very large background absorption from the associated protein and water. 
The high-spectral resolution of the superconducting tunnel junction detectors provides a way to measure the weak fluorescence from transition-metal L lines which would otherwise be buried in the wings of much stronger neighboring lines. For example, manganese in biological molecules, such as the photosynthetic, oxygenevolving complex, has weak fluorescence lines at 637 and 649 electronvolts that are obscured by the strong oxygen $\mathrm{K}$ emission line at 525 electronvolts. The results of this work generated much interest in the scientific community and were featured in the March / April 2001 issue of Synchrotron Radiation Neros (Fig. 38).

\section{High-Power Lasers}

The High Power Laser Group (HPLG) in I Division is working on several projects that are aimed at both developing new high-power laser sources and novel applications that use high-power lasers. The largest project is a spin-off from the Atomic Vapor Laser Isotope Separation (AVLIS) Project for Uranium nuclear fuels. The project uses a suite of tuned lasers to isotopically separate materials of importance for the DOE Stockpile Stewardship Program. New technologies for this project include high-power, diodepumped, neodymium-doped ( $\mathrm{Nd}$ :) yttrium aluminum garnet (YAG) solidstate lasers; frequency doubled to pump tunable dye lasers; and a set of nonlinear, solid-state lasers for the master oscillators. It is expected that these laser systems will serve as prototypes for other projects, such as medical laser isotope separation and artificial guide star systems for astronomy and missile defense.

Other projects that use high-power lasers included an effort to launch strong shock waves in metallic materials and an investigation into the metal-working capabilities of diode-pumped, solid-state lasers (DPSSLs). The Laser Shock Diagnostic (LSD) project uses a high-energy, nanosecond-pulse laser to study materials that are important to the DOE Stockpile Stewardship Program in a much less expensive way and with higher data rates

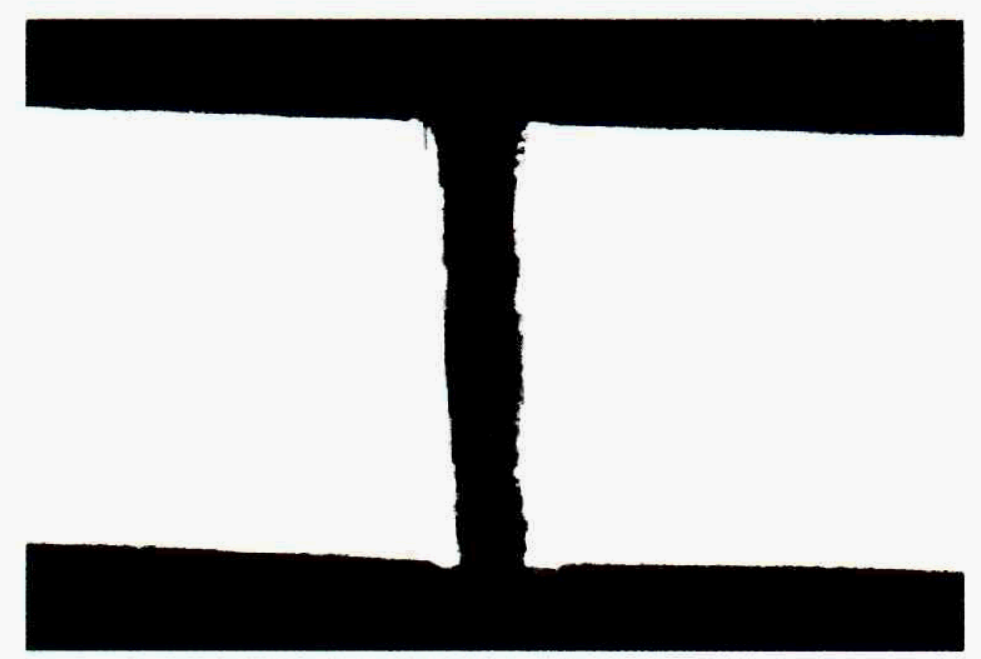

than have been previously possible using other technologies. Pending a successful demonstration of the LSD technique with existing lasers, the HPLG will construct a new dedicated laser facility and conduct significant follow-on experiments.

The metal-working effort has shown that precision drilling of submillimeter holes in millimeter-thick substrates can be accomplished using a relatively inexpensive DPSSL with a pulse length in tens of nanoseconds as opposed to complex lasers utilized for ultrashort (subpicosecond) laser processing. The major requirement for these precision holes is the absence of any heat-affected zone (HAZ), which could compromise material properties. Results (shown in Fig. 39) indicate that if longer pulses are used with incident fluxes just above the ablation threshold, no HAZ is present. The technique that the HPLG is testing uses a two-color, two-mode approach to rapidly drill an undersized hole and then polish and shape the sides to final dimensions.

The HPLG is also developing new highpower laser sources and related technologies. A new liquid laser concept is being investigated (using LDRD funds) that combines the superior properties of $\mathrm{Nd}$ : glass lasers with the high-average-power capabilities of circulated, organic liquid dye lasers. Spectroscopic and fluid dynamic modeling studies indicate that near-diffraction, limited-quality beams in the tens of kilowatts average power range can be achieved from a single aperture with the possibility of combining several such beams to produce total powers at a fraction of a megawatt.
Fig. 39. Shown at 50 times magnification is a 200-micron-diameter hole drilled in a 1.25millimeter stainlesssteel plate by a laser operating at 235 watts with 85-nanosecondduration pulses of 1064-nanometer light. Drilling time was less than 1 second. The existence of grain boundaries contiguous to the hole indicates the absence of a heat-affected zone. The slight amount of material at the top of the hole is recast metal that would be removed in a second, polishing phase of the process. 


\section{Advanced Interceptor Technology Pr}
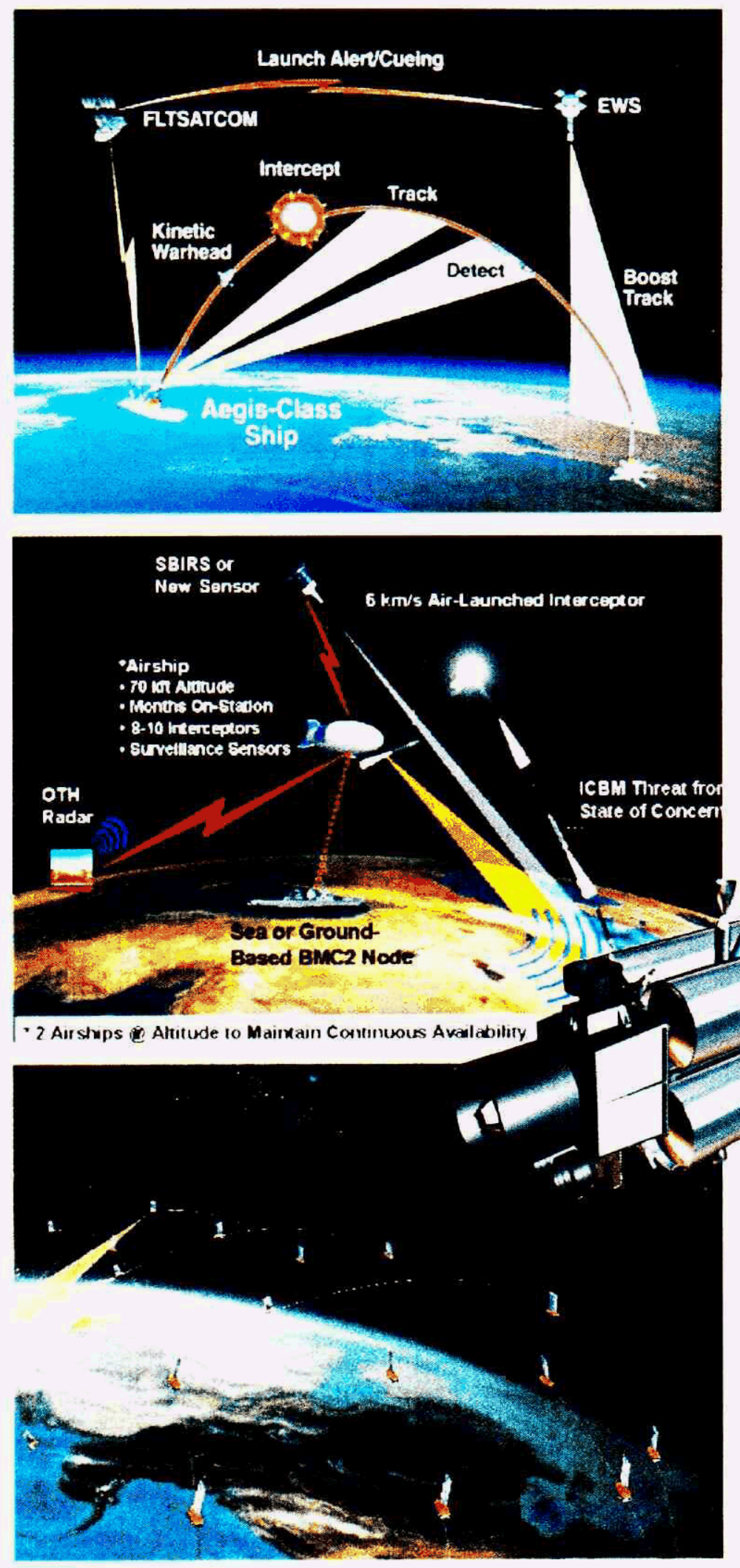

Fig. 40. The Advanced Technology Kill Vehicle's delta- $v$ of 1.5 to $2.5 \mathrm{~km} / \mathrm{s}$ is enabling for all boost-phase intercept basing modes.
The research and engineering focus of the Advanced Interceptor Technology Program (AITP) is in areas of high national importance, including developing, integrating, and demonstrating the critical technologies for performing hypersonic, hit-tokill intercepts of theater ballistic missiles just outside the atmosphere. The work of AITP involves space technologies for space defense purposes, analysis of National Missile Defense options for the U.S. Department of Defense (DoD), and the development of advanced technologies to improve boost and mid-course ballistic missile defense capabilities. It is conducted under the sponsorship and in coordination with the Missile Defense Agency (MDA), formerly the Ballistic Missile Defense Organization (BMDO), and the U.S. Navy.

In the past 18 months, AITP has successfully developed a concept for a high-performance interceptor for use in a broad range of missile defense programs. Known as the Advanced Technology Kill Vehicle (ATKV), this concept advances technologies originating from prior Advanced Interceptor Technology programs, including those to develop a high-performance "microsatellite." Over this period, AITP established unique capabilities in three areas:
- Propulsion systems for small vehicles that can perform space missions

- Integrated sensing for proximity operations, including the guidance and control software necessary for docking in orbit and intercepting a missile

- Ground-testing capabilities for demonstrating, characterizing, and calibrating the performance of integrated systems and subsystems.

In addition, in coordination with Johns Hopkins University's Applied Physics Laboratory, AITP has supported the DoD in the production of a classified analysis of the U.S. Navy's capabilities to support National Missile Defense and in the development of alternative system concepts to expand the approach to missile defense. Work is also underway for both MDA and the U.S. Army's Strategic Missile Defense Command (SMDC) to evaluate how the ATKV can support several different boost-phase defense system concepts. Fig. 40 shows sea-based, air-based, and space-based ballistic missile defense concepts and how the unique features of the ATKV, especially its high delta-v, are enabling for these applications. Other proposed developments of the ATKV include a modernized Brilliant Pebble-like kill vehicle that is both lightweight and high performance and has both boost-phase intercept and mid-course engagement applications.

\section{Relevance to National Needs and Agency Missions}

The ultimate measure of this work's value is its adoption by the current Administration as a baseline technology for several missile defense options. The national importance of the work has been demonstrated by direct Congressional funding of LLNL's efforts and the provision of program early-start funding in Fiscal Year (FY) 2000 by MDA and the Navy. DOE has concluded a Memorandum of Agreement with the DoD on the use of DOE defense 


\section{ogram}

laboratories to support missile defense work. In turn, MDA designated the ATKV work last June as one of its major "cooperative" efforts with DOE on ballistic missile defense and briefed House and Senate Authorization and Appropriations Committees on the ATKV work.

ATKV continues to receive Congressional support in terms of FY2001 "add-on" funding. Strong support is expected in FY2002 for an early Advanced Concept Technology Demonstration.

\section{High-mass Fraction Propulsion System Specific AITP achievements in 2001 included the development of a high- performance pump for use with hydrogen peroxide as a monopropellant; the identi- fication of requirements for an "inte- grated" system, including mass allocations; and the preliminary system design and identification of critical mile- stones. The propulsion system advances the state of the art by providing a "high- mass fraction" system that is capable of "thrust on demand" using "safe" propel- lants. A preliminary, pressure-fed, ground-test vehicle prototype has been developed for propulsion testing at AITP's ground-test facility; and the unique swivel thruster feature of this vehicle design has been demonstrated.}

\section{Docking Experiments}

AITP advanced the capability to ground test the flight performance of an interceptor during "end-game" maneuvers or of a microsatellite during docking maneuvers. This ground test provides a unique, 5-degrees-of-freedom test that simulates a microgravity environment. This allows the performance evaluation of a microsatellite or interceptor against a target vehicle in a realistic test environment that enables the validation of simulation models for expected performance. Fig. 41 shows two ground-test microsatellite test vehicles that have been used to perform a series of docking experiments and demonstrations.
The lower vehicle maneuvers to the upper vehicle, docks with it, and then tows it back along the table. The value of this unique approach is reliable testing at a low cost, the dramatic increase in the number of tests that can be performed (hundreds of runs versus a few), and the high output of flight code. Essentially, three employees can develop the code that just ten years ago required the efforts of ten times that many. With respect to flight testing, a traditional "hover test" would cost $\$ 2$ to $\$ 3$ million. With the LLNLdeveloped approach, the cost is that of the six full-time employees necessary to operate the test capability. This facility has been so successful that the DoD is interested in expanding the Laboratory's experimental capability by developing an enlarged test table, outdoor rail, and associated instrumentation.

The ATKV program is developing a monopropellant, pump-fed test-bed vehicle that will be used as an end-to-end proof of concept of a highmass fraction interceptor system. Development of miniaturized pumps and propulsion system components (thrusters, valves, and tanks) will be integrated with upgraded control electronics for this test-bed vehicle. Integrated vehicle tests are planned to start in FY2002, including onsite, low-cost "hover tests" later in the year.
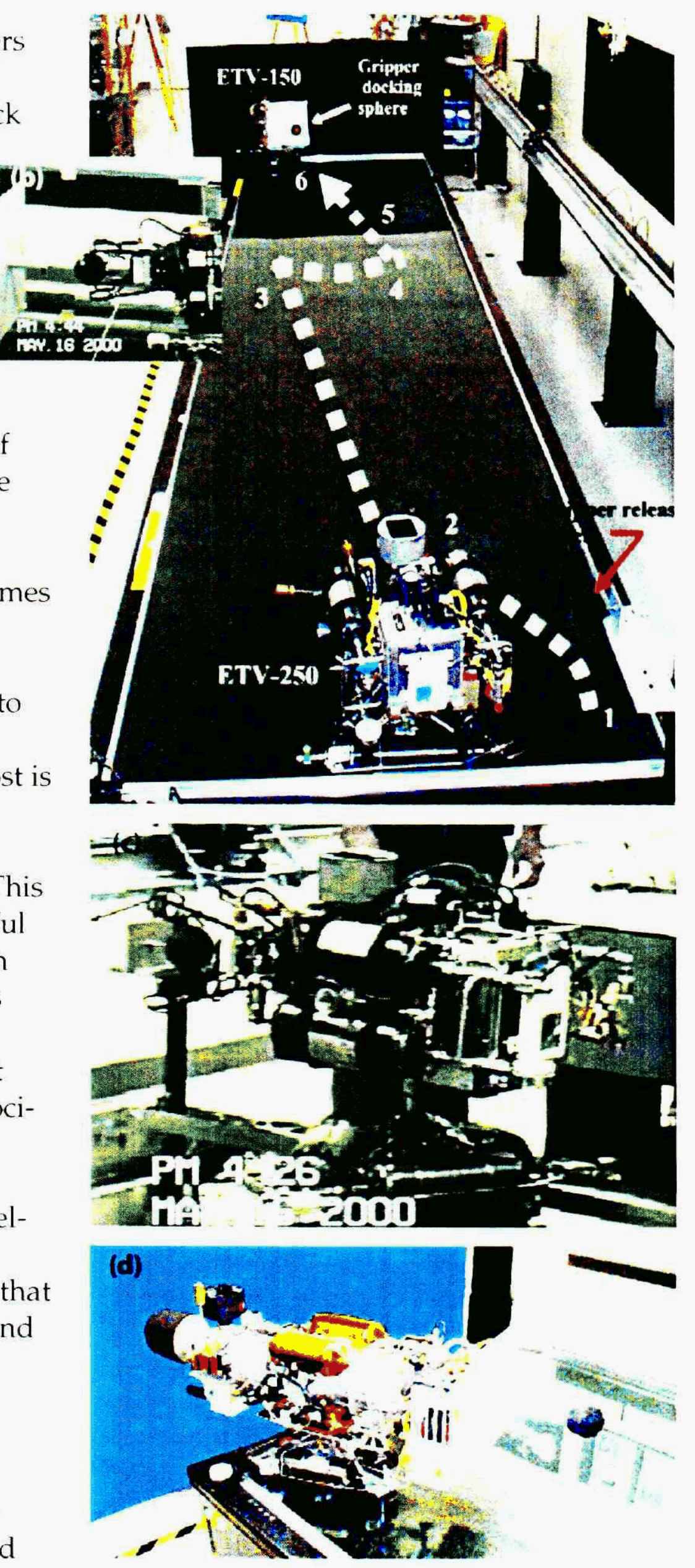

Fig. 41. Two ground-test micro-satellite test vehicles are used in a docking demonstration at AITP's indoor ground test facility. The bottom vehicle in photo (a) docks with and tows the upper vehicle. Insert (b) shows the docking. Photos (c) and (d) show the gripping and target vehicles, respectively. 


\section{Laser Science and Technology Progr}

The mission of the Laser Science and Technology (LS\&T) Program is to develop advanced solid-state lasers, optics, materials technologies, and applications to solve problems and create new capabilities of importance to the nation and the Laboratory. A top, near-term priority is to provide technical support and core competencies to the NIF Programs Directorate to ensure the successful commissioning of the facility. The LS\&T Program devotes special effort to building and maintaining its capabilities in three technology areas: highpower, solid-state lasers; high-power optical materials; and applications of advanced lasers.

The PAT Directorate exercised management oversight of several LS\&T technical efforts. Only those projects with close PAT involvement are described here. LS\&T Program activities for the NIF are more completely discussed in publications from the NIF Programs Directorate. For example, other LS\&T projects not covered here include:

- Extensive details on technology and optical component development to improve the lifetime of 3-omega ultraviolet (UV) optics and enhance the NIF's operations and performance capabilities

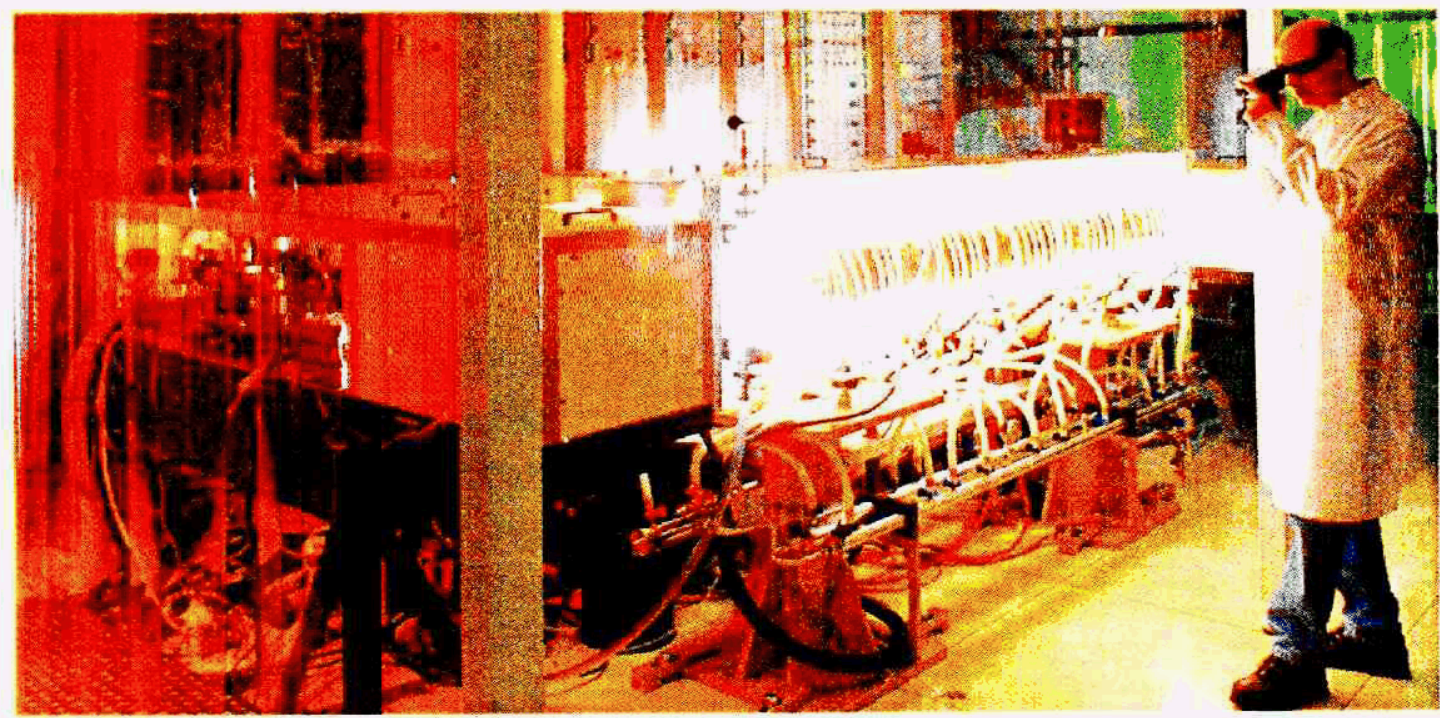

Fig. 42. The 10-kilowatt laser system, developed under U.S. Army funding for missile defense, was installed at the High Energy Laser System Test Facility (HELSTF) at the White Sands Missile Range, New Mexico.
- Complete construction and test of the Z-Beamlet and the Laser-Trigger Systems at SNL

- Kilowatt-to-megawatt-class, solid-state laser technology for inertial fusion energy

- Use of laser peening to increase the corrosion resistance of metal

- Short-pulse, high-average-power, solidstate lasers for micromachining

- Meter-scale diffractive optics for petawatt lasers

- New wet-etching process for figuring large-aperture and ultrathin optics

- Lightweight diffractive optics for space.

\section{Development of a Solid-state, Heat-capacity Laser for the Department of Defense}

Under the support of the U.S. Army's SMDC, and in collaboration with industrial partners (Raytheon, Litton, and others), the LS\&T Program is developing high-average-power (100-kilowatt-class), diode-pumped, solid-state, heat-capacity laser technology for application in tactical, short-range air defense missions. The ultimate vision is an electrically powered, diode-pumped, solid-state laser weapon that can be deployed on an electric vehicle. To establish a solid technical basis for the heat-capacity laser operation and risk reduction, two flashlamp-pumped, $\mathrm{Nd}$ :glass laser prototypes-a three-disk and a nine-disk module-were built and tested.

\section{Flashlamp-pumped, Nd:Glass Laser Prototypes}

In 2001, the construction and testing of the nine-disk module were completed. This module is designed to produce 10 kilowatts of average output power and deliver laser pulses with beam quality less than three times diffraction-limited and 500 joules of energy per pulse at a repetition rate of 20 hertz for 10 -second bursts. The laser was delivered to the Army's High Energy Laser System Test Facility 
(HELSTF) at the White Sands Missile Range, New Mexico, to support material interaction testing (Fig. 42).

In August 2001, heat-capacity operation for a full 10 seconds with an average power of 13 kilowatts (639 joules of energy per pulse at 20 hertz) was successfully demonstrated. Currently, the laser is operating with a stable resonator, which is ideally suited for the target interaction experiments. Using a stable resonator with an output coupling of $29 \%$, up to

1,000 joules of energy per pulse were extracted from the amplifier. A beam quality of approximately 3.5 times the diffraction limit was demonstrated with an unstable resonator and with no deformable mirror (DM) correction. Components that will improve beam quality - such as the use of a deformable mirror-include DM control electronics and laser-diagnostic sensor packages that have been assembled and tested. With the new DM and an unstable resonator, this laser can achieve the desired goal of two times the diffraction-limit beam quality over the entire 10 -second run time.

Using this nine-disk prototype heatcapacity laser, LS\&T researchers conducted a series of target interaction experiments, operating the heat-capacity laser at 3 hertz with an energy of 650 joules per pulse. The laser-beam footprint was roughly square with a 6-millimeter-by-6.5-millimeter spot dimensions at the target. The irradiation pulse had a temporal envelope of 300 to 400 microseconds and consisted of several relaxation-oscillation spikes with peak intensities near a megawatt-per-square centimeter. About 0.9 cubic centimeters of aluminum (11 millimeters deep) were successfully removed after irradiation with 38 laser pulses. For future target interaction experiments, development continued of numerical models to simulate the material-removal process using hydrodynamics codes to model the vaporization and material ejection process. During a ribbon-cutting ceremony at the HELSTF, the nine-disk laser was fired at a 1-inchthick aluminum target and successfully penetrated the target in 2 seconds (Fig. 43).

\section{Diode-pumped, Nd:GGG Crystalline Laser}

The development of a 100-kilowatt-class, diode-pumped, $\mathrm{Nd}$ :gadolinium gallium garnet (GGG) crystalline laser is proceeding rapidly. The diode-pumped Nd:GGG heat-capacity laser will have better thermal characteristics and operating efficiency, allowing the output power to be scaled to 100 kilowatts (500 joules of energy per pulse at 200 hertz). Significant progress was made toward the growth of highquality Nd:GGG boules. Litton-

Airtron Synoptics is now routinely growing Nd:GGG boules measuring 15 centimeters in diameter with high optical quality.

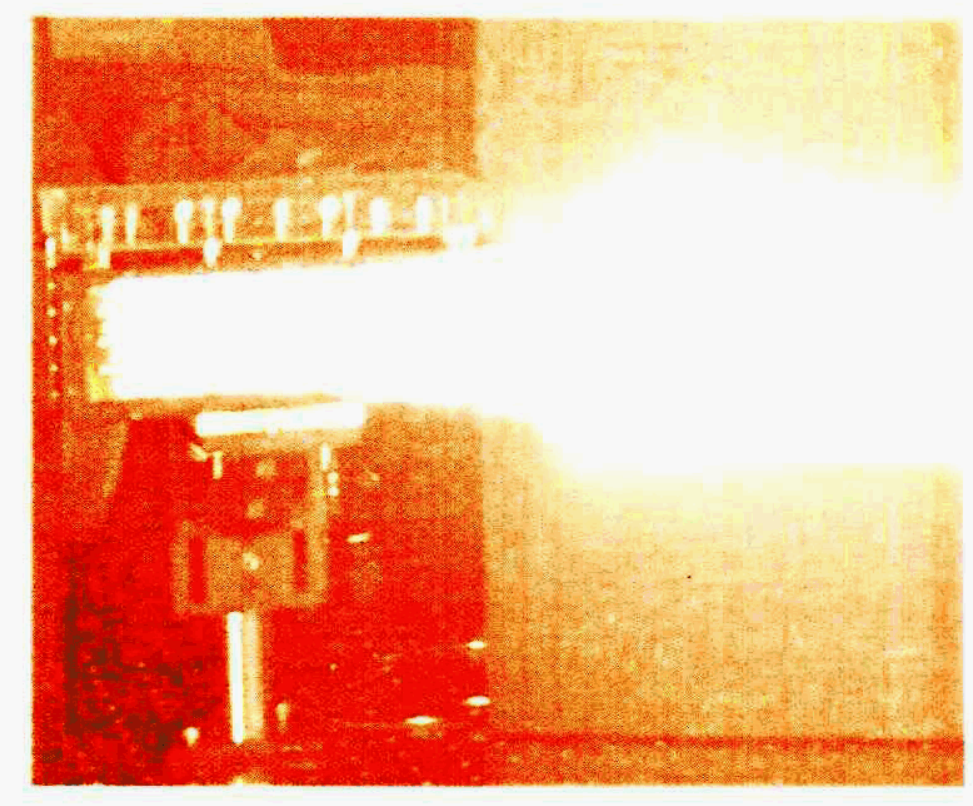

Also completed were the fabrication and the testing of a 42-kilowatt laser-diode array for pumping Nd:GGG slabs. Shown in Fig. 44, this 100-kilowatt-class diode module is constructed from 280 edge-emitting laser-diodes mounted on 28 closely packed, silicon microchannel, monolithic (SiMM) tiles arranged in $4 \times 7$ configuration. Each individual tile holds ten diode bars. The brightness of the array is approximately 1 kilowatt per square centimeter at a $10 \%$ duty factor. In order to fully utilize the light emitted from laser-diode bars, microlenses were designed to collect radiation along the fast axis and were able to reduce the beam divergence angle from 30 degrees to less than 1 degree.

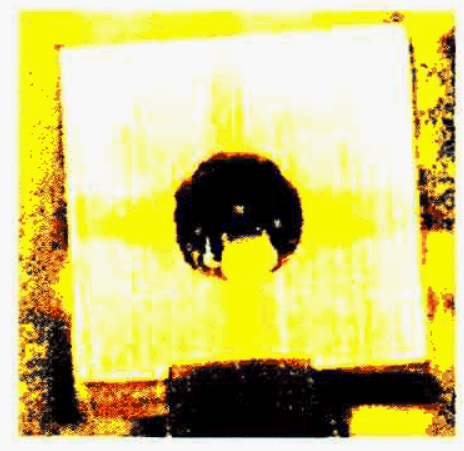

Fig. 43. A 1-inch-thick aluminum target with a hole drilled by a 2-second laser burst from the HELSTF laser.
Fig. 44. A 100-kilowattclass diode array module in operation. It was developed for pumping high-averagepower, solid-state lasers. 


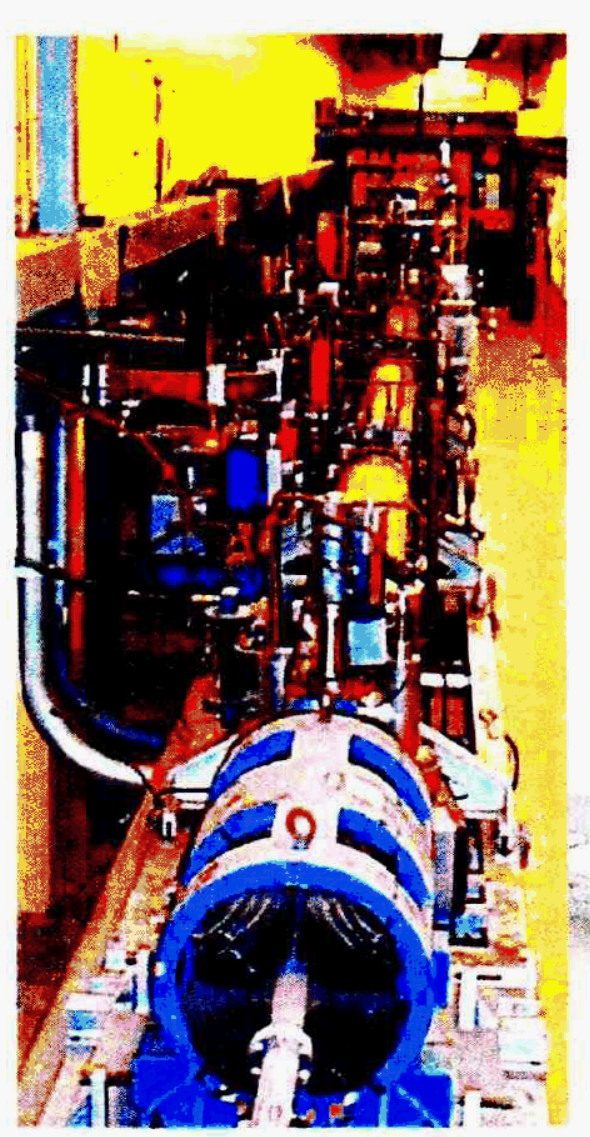

\section{High-energy, Ultrashort-pulse Laser Technology Development}

With support from the LDRD program, LS\&T recently developed a new frontend technology that utilizes opticalparametric, chirped-pulse amplification (OPCPA) to replace the regenerative amplifier used in a variety of chirpedpulse amplifiers, including the Nova Petawatt Laser, Falcon Laser, and other short-pulse laser systems. The wavelength

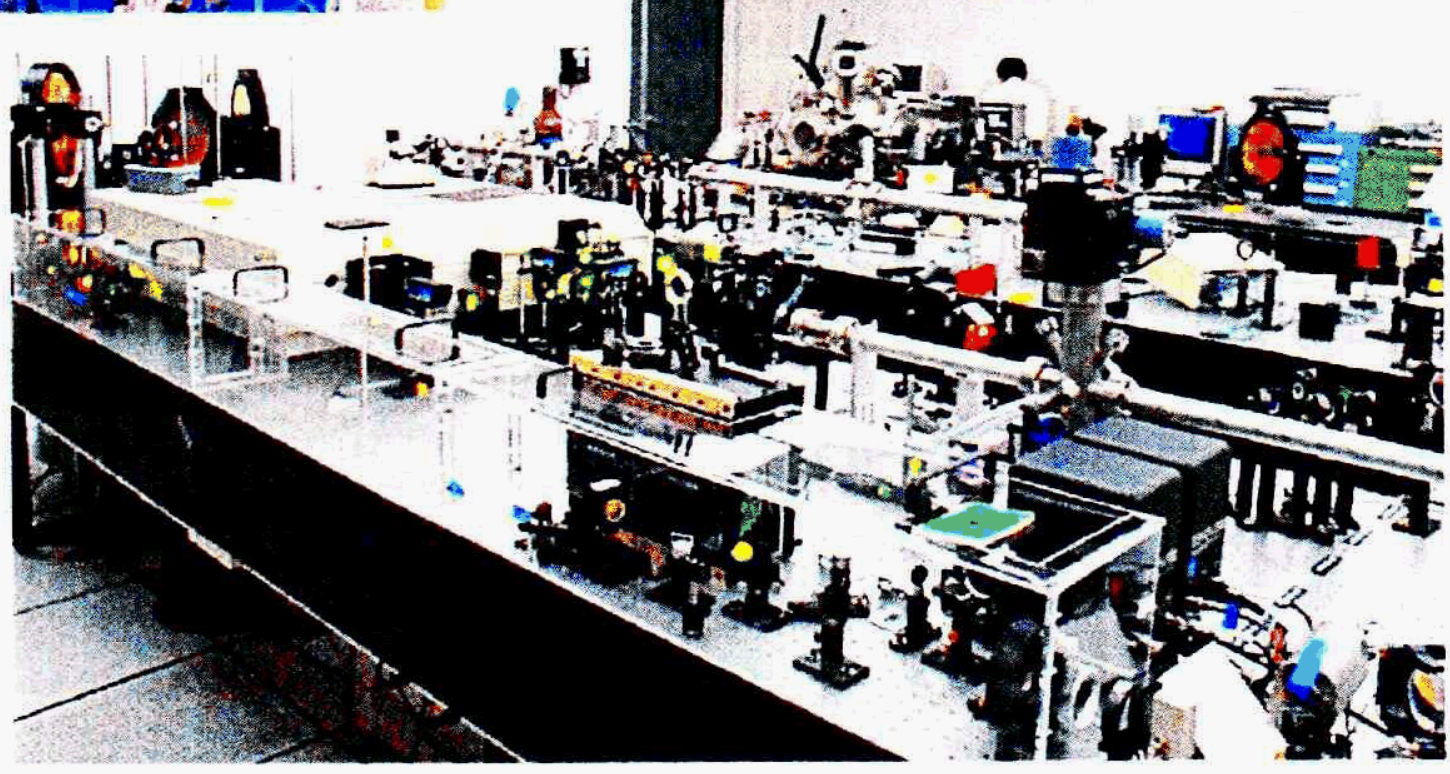

Fig. 45. The Falcon ultrashort pulse laser (above) and the LINAC (above left) are being integrated to produce a unique ultrafast $\mathrm{x}$-ray source for Stockpile Stewardship Program experiments. and pulse-energy levels obtained from this OPCPA are ideal for seeding kilojoule-class $\mathrm{Nd}$ :glass amplifiers, such as the NIF and other high-energy, ultrashort-pulse laser systems.

Previously, high-energy lasers employing chirped-pulse amplification (CPA) were built on a hybrid (Ti:Sapphire-Nd:glass) laser technology. Two difficulties with the hybrid laser system are its low pulse contrast and the generation of prepulses by the regenerative amplifier. With a regenerative amplifier, a tiny amount of energy leaks out with each round trip of the laser pulse. If this leak, or prepulse, is not attenuated, it may cause a preplasma, which changes the coupling of the laser to the target. Single-pass OPCPA eliminates prepulses originating from regenerative amplifiers and reduces the amplified spontaneous emission (ASE) by approximately one order of magnitude compared to Ti:Sapphire. OPCPA offers additional advantages when compared to regenerative CPA, including low thermal aberration, greater wavelength flexibility, higher gain, high-beam quality, and lower B-integral as a result of the short beam path through the gain medium.

In 2001, the LS\&T Program built a compact OPCPA using a commercial, frequency-doubled, Q-switched Nd:YAG laser as the pump source. Three betabarium borate (BBO) crystals were used as the gain medium. Under the type I angular phase-matching condition, two crystals were configured as preamplifiers; and one was used as the power amplifier. LS\&T investigators successfully demonstrated high-gain (approximately $10^{7}$ ), high-conversion efficiency (more than $20 \%$ ), good amplified pulse beam quality $\left(M^{2}<2\right)$, and high-bandwidth fidelity in the OPCPA (30 femtoseconds).

Widespread use of the OPCPA is anticipated as the frontend technology for glass-based, petawatt-laser systems and high-average-power systems. In the future, it may be possible to exploit the full band width capability of OPCPA to produce kilojoule-class pulses with a 
duration of several tens of femtoseconds. This would potentially allow focused intensities of $10^{24}$ watts per square centimeter or higher for experiments at extreme fields.

For the Stockpile Stewardship Program, LS\&T researchers continue to study the ultrafast response of materials. An ultrafast $x$-ray source was developed, based on Thomson scattering of femtosecond laser pulses (i.e., the Falcon Laser) from 100-megaelectronvolt relativistic electron bunches. During 2001, the multiterawatt, ultrashort-pulse Falcon Laser system and the Linac were integrated (Fig. 45).

Several techniques were tested for timing synchronization between the electron bunch and laser pulse to approximately 2 -picosecond accuracy. The Linac was reconfigured for installation of a 5-megaelectronvolt photoinjector.

Considerable advances were also made to the Falcon Laser and the photoinjector laser systems. LS\&T researchers succeeded in demonstrating Thomson scattering with the photoinjector and generated 600-electronvolt $x$-ray photons by scattering off the 5-megaelectronvolt electron beam with the 400-femtosecond laser pulses from the Falcon. The installation of a new final power amplifier increased the Falcon Laser output fourfold.

A new front end was developed for the Falcon Laser, based upon a hybrid system using both OPCPA and Ti:Sapphire lasers. The hybrid design enabled the pump efficiency and the energy of the compressed pulses to be increased. Integration activities are planned for completion in 2002.

When finished, this ultrafast $x$-ray source will be used to probe the dynamics of shock propagation and phase transitions in high- $Z$ metals.

\section{Laser Guide Star Systems for Ground- based Observatories}

The LS\&T Program continues to collaborate with other Laboratory programs to develop fieldable, sodium-layer laser guide star systems for use on large astro- nomical telescopes in the Lick Observatory near San Jose, California, and at the Keck Observatory in Hawaii.

The use of a laser guide star at 589 nanometers, makes possible the creation of artificial stars in the direction of observation by utilizing laser-induced fluorescence in the mesospheric sodium layer 90 kilometers above the earth's surface. Using laser guide star beacons allows diffraction-limited images to be collected from more than $60 \%$ of the sky. During the past year, LS\&T researchers developed, installed, and activated compact, robust, and reliable laser guide star and adaptive optic systems at the Lick and the Keck Observatories. These laser guide stars - using frequencydoubled Nd:YAG lasers to pump a dye oscillator, preamplifier, and amplifierroutinely produce up to 20 watts at 11 kilohertz with $1.5 \%$ energy stability and a Strehl ratio of 0.647 . The laser guide star system is currently being used for regular and routine science observing at the Lick Observatory. The Keck system has completed performance and atmospheric propagation tests. (See Fig. 46 showing the sytem in operation at the

Fig. 46. The LLNL-built orange laser beam of the virtual guide star emerges from the dome of the Keck II telescope atop 14,000foot Mauna Kea volcano in Hawaii. (photo by John McDonald, CanadaFrance-Hawaii Telescope Corporation)

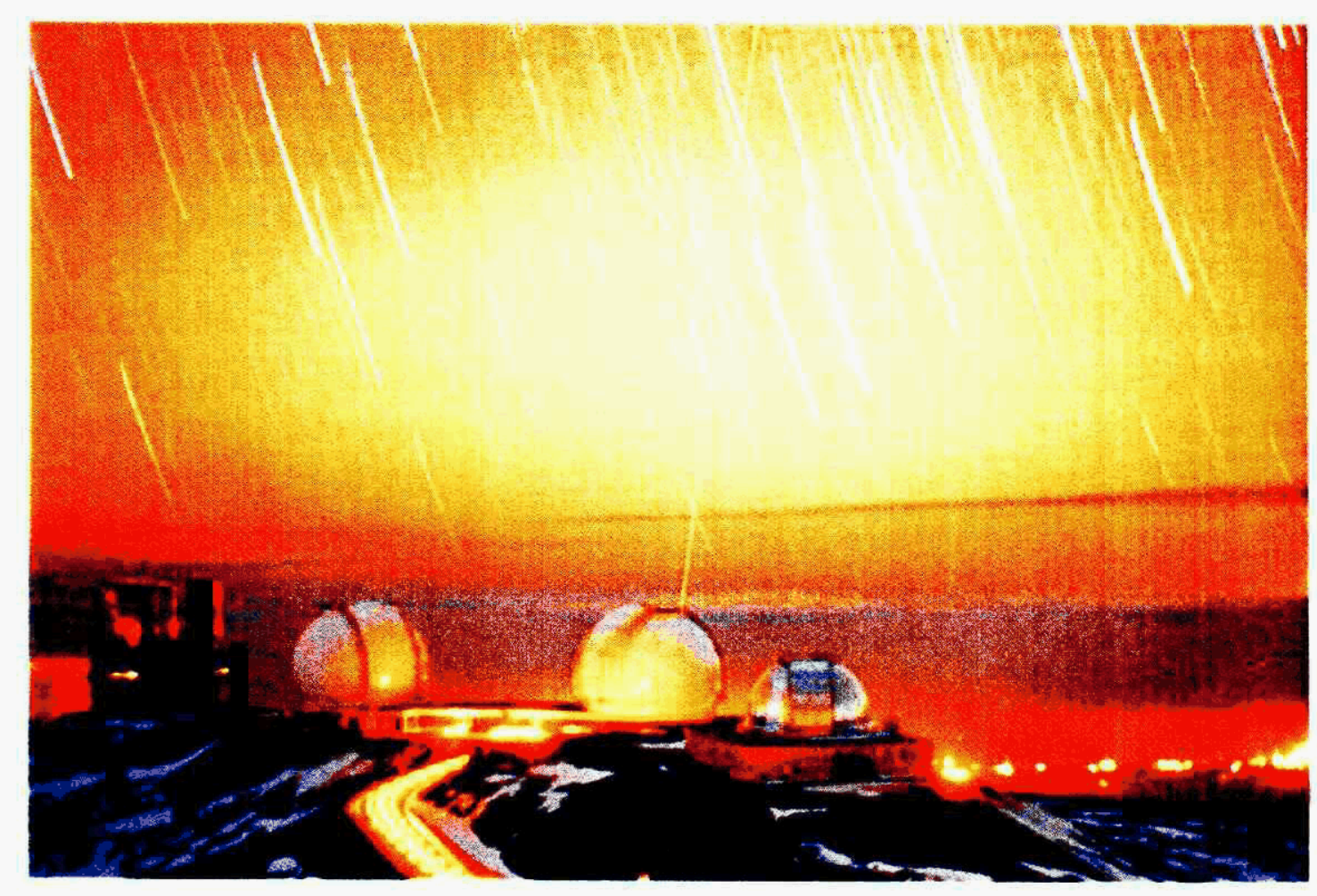

Keck Observatory.) An all-solid-state fiber guide star laser for next-generation telescopes is being developed. 


\section{Institute for Laser Science and Applic}

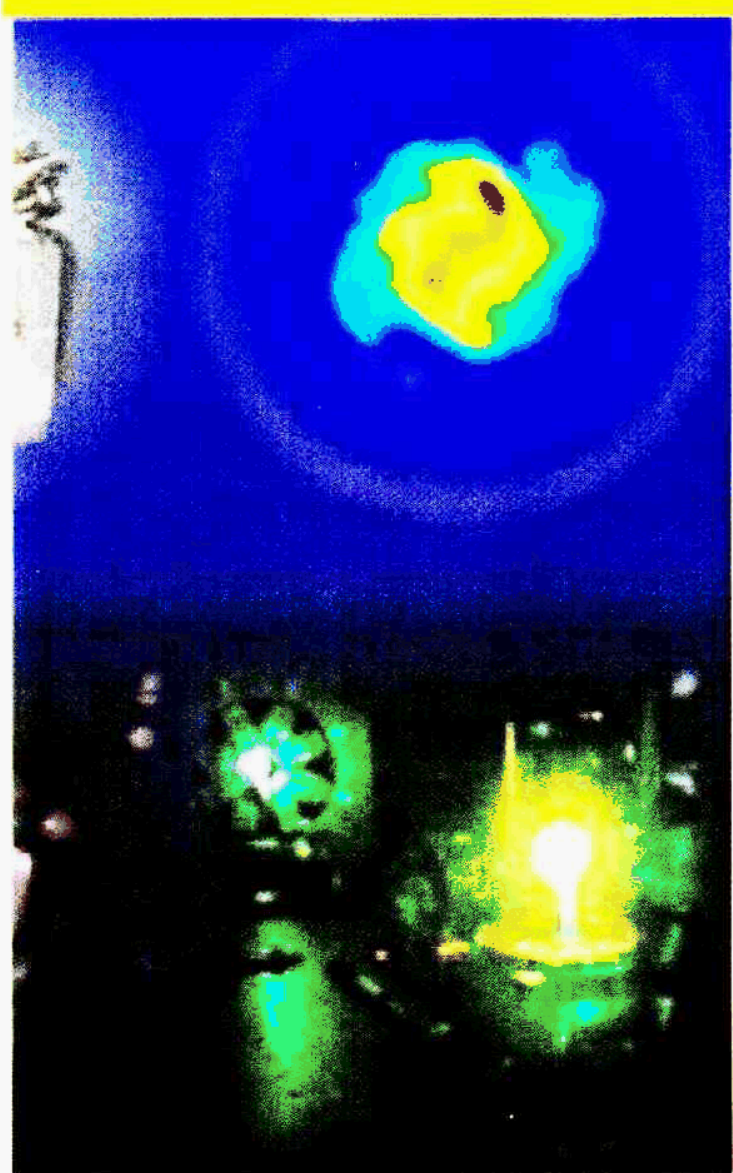

Shown in this composite photo are an angular map of $x$-rays produced by Compton scattering (top) and an 8-pass titanium: sapphire amplifier (bottom).
The Institute for Laser Science and Applications (ILSA) supports projects on the application of high-intensity, highenergy lasers in areas that include laserplasma physics, high-brightness and high-energy particle beam applications to novel x-ray sources, ultrashort-laser-pulse interaction physics, and the study of matter under extreme conditions.

Created in 1996 under the University Relations Program (URP), the Institute has had the mission to facilitate training and research for university students and faculty in areas important to DOE in laser-interaction-with-matter science and advanced laser technologies. As part of this mission, the Institute oversees access for students and faculty to existing LLNL experimental facilities. This activity assists and enhances the scientific environment at LLNL and creates a forward-looking research effort that provides strategic benefit to both the scientific and the university communities. ILSA's solid program of outreach to the university community includes the University Collaborative Research Program (UCRP) and the organization of conferences and workshops in topical areas of common interest.

Plasma-induced Laser Incoherence An important focus for plasma physics is high-intensity laser plasma interactions under a number of plasma conditions, including those for Inertial Confinement Fusion (ICF) plasmas. The propagation of intense laser pulses through underdense, fully ionized plasmas can be affected by a number of nonlinear processes, including filamentation, self-focusing, or parametric instabilities, such as stimulated Brillouin and Raman scattering. Understanding these processes is important because they can be detrimental to the coupling of laser energy to targets and the modifying of propagation characteristics. The problem of laser coupling to a target or plasma is a concern in many projects related to the Stockpile Stewardship Program, including ICF.

Last year, ILSA started a comprehensive program in collaboration with colleagues, both experimental and theoretical, to study how these nonlinear processes modify the laser propagation itself. The induced spatial and temporal incoherence of the laser beam - the so-called selfinduced plasma smoothing (SIPS) - has to be considered if parametric processes in laser plasmas are to be understood. Recent theoretical studies have suggested that the propagation of the laser beam through the underdense plasma could also induce spatial and temporal incoherence upon an already spatially randomized laser beam that utilizes a random phase plate (RPP). The SIPS process would develop through the nonlinear coupling between stimulated Brillouin forward scattering (SBFS) and the dynamically evolving hot spots of the beam. SIPS could not only influence laserplasma interactions but also interfere with the externally applied smoothing techniques, thus modifying their expected performances.

Experimental results demonstrated that SIPS does significantly modify the properties of an intense propagating beam. Fig. 47 shows the modification to the transmitted laser light in the presence of a preformed plasma. Filamentation and 


\section{ations}

SBFS are the main processes that can couple the incident laser light with the plasma and induce large-angle spreading and large red spectral shifts. Numerical simulations that have a similar selffocusing-filamentation initial stage display, at later propagation times, an unsteady behavior of the plasma response with strongly deflected filaments oscillating in and out of existence. This oscillation is primarily caused by the mixing between the density wells dug by neighboring filaments. Such a rapidly bifurcating motion of the filaments decorrelates the light from the plasma response and smoothes the spatial intensity distribution. In addition, at high intensity, these simulations also display large red shifts and spectral broadening for the forward scattered light.

This work was carried out in collaboration with other LLNL colleagues, the Laboratory for Laser Energetics of the University of Rochester, the University of Alberta, and École Polytechnique in Palaiseau, France.

\section{Fluctuations and Nonlinear Waves in Laser-produced Plasmas}

Thomson scattering (TS) from stable electron density fluctuations is a powerful diagnostic tool that is used to measure local plasma parameters, distribution functions, and transport processes. In laser-produced plasmas, TS has become a standard technique for studying enhanced fluctuations and related instabilities. The TS cross section is determined by the dynamical form factor for electron density fluctuations $S(k, w)$. The evaluation of the dynamical form factor requires the solution of equations of motion for the density fluctuations in the plasma. An ongoing development of the particle code, which includes particle collisions and the interaction with an oscillatory electric field, resulted in a new,

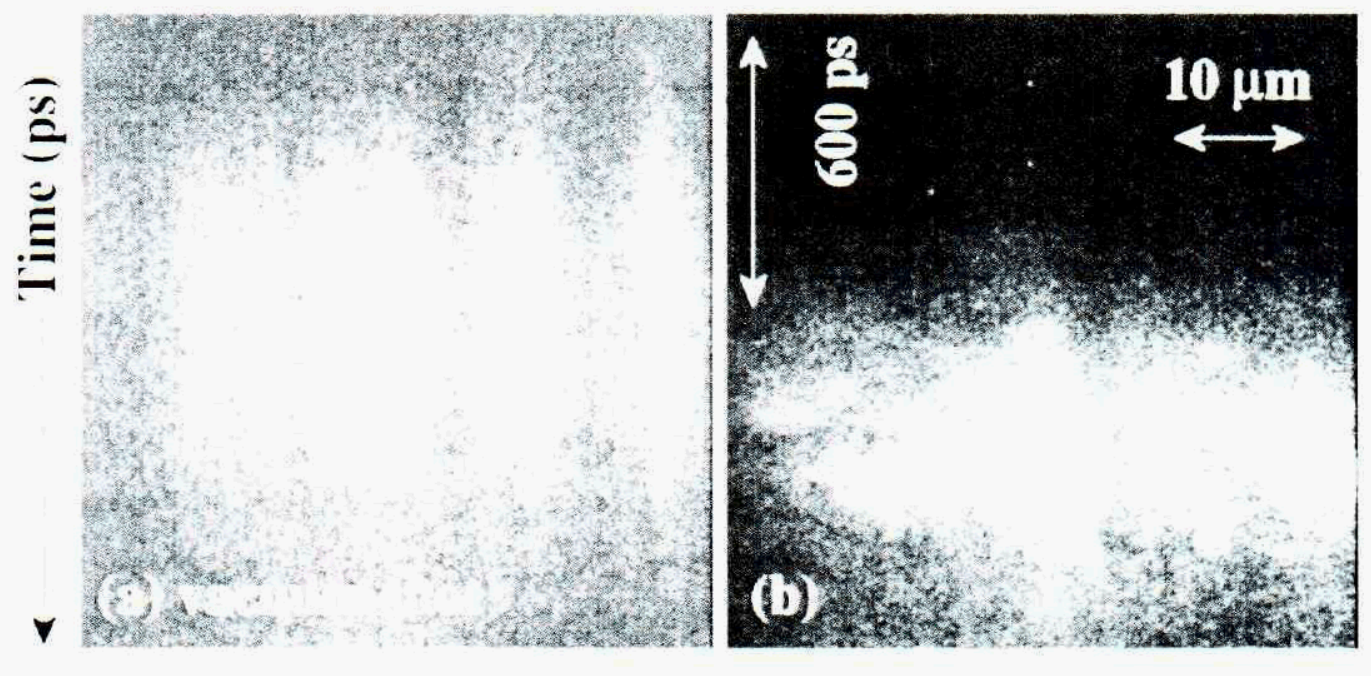

nonMaxwellian distribution function and in preliminary results involving nonlinear thermal transport properties.

In this project, ILSA developed a theoretical framework that combined calculations of $S(k, w)$ with hydrodynamic evolution of laser-produced plasmas and that accounted for the experimental resolutions of measuring devices. The theory leads to accurate predictions for the TS cross section that, after comparison with experimentally measured scattered power, has elucidated new kinetic processes in high-Z gold plasmas.

Studies of high-Z plasmas improve understanding of radiative properties of hot dense matter and have many applications in ICF, laboratory astrophysics, and $x$-ray lasers. This theory has been applied to the interpretation of TS data in gold plasmas. In this experiment, ion acoustic and Langmuir wave fluctuation spectra were simultaneously observed for the first time, allowing accurate measurements of plasma parameters and, in particular, an average ionization stage.

\section{Plasma Focusing of 30-gigaelec- tronvolt Electrons and Positrons}

An important contribution last year in the area of laser plasmas was the use of a laser-produced plasma as a lens to focus gigaelectronvolt electrons and positrons. The plasma lens has been proposed as a
Fig. 47. One-dimensional, time-resolved, near-field images of the forward scattered light at $0 \pm 10^{\circ}$. (a) Stationary speckle pat tern of $f 13$ incident light (vacuum shot). (b) Through a plasma of electron density $\sim 20 \%$ critical and with an intensity $I=3 \times 10^{14}$ watts per square centimeter. 
Fig. 48. Plasma focusing data for positron beams provided an important contribution to the study of laser plasma.

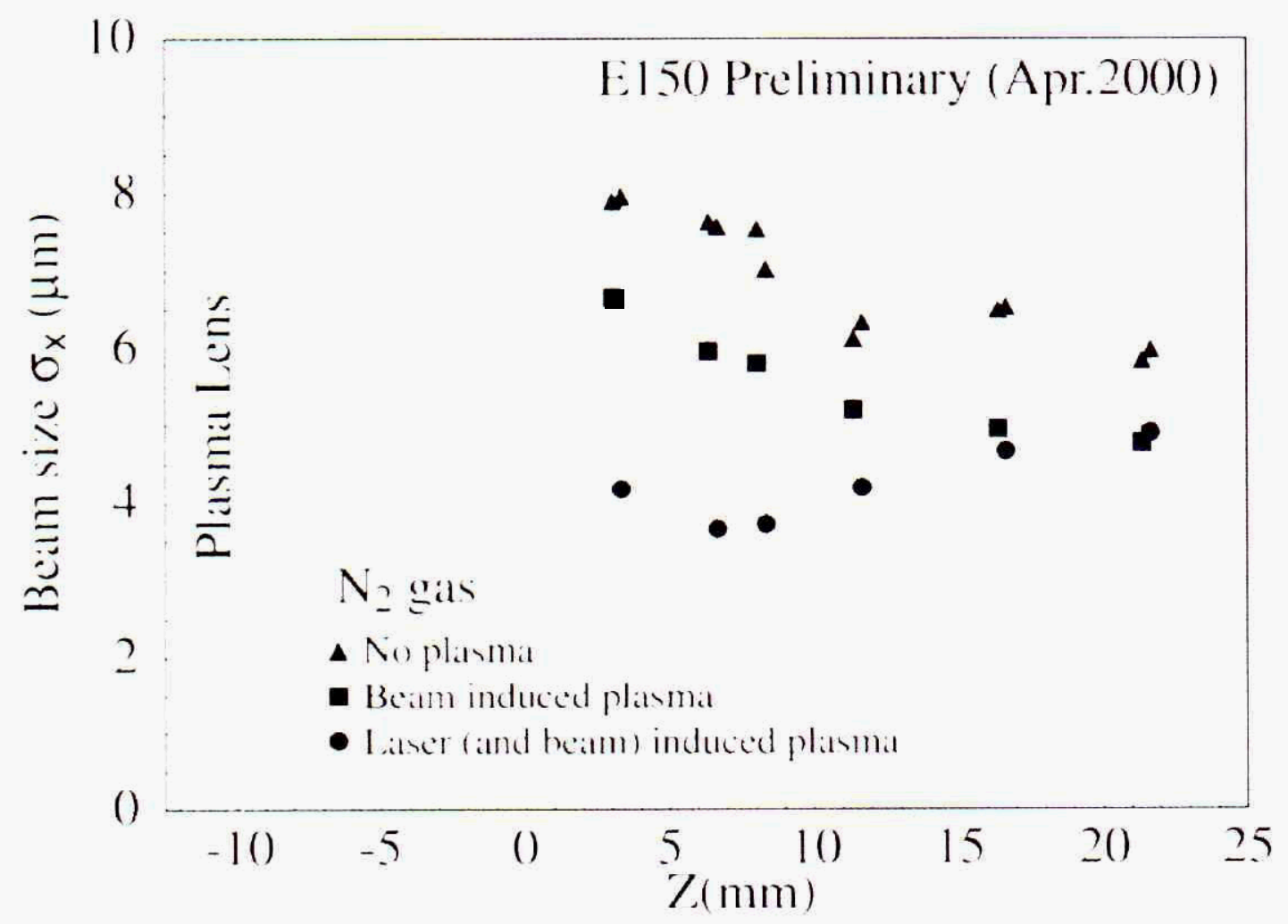
including those encountered in the Stockpile Stewardship Program. ILSAbased calculations and theoretical analysis first demonstrated the potential use of an existing $x$-ray laser for probing highdensity plasmas, including those approaching solid densities. colliders. The experiment was carried out at the SLAC on the Final Focus Test Beam facility. The experiment clearly demonstrated the focusing of 30 -gigaelectronvolt electrons and positrons. Fig. 48 shows the focusing of the positron beam by a plasma, formed either with the aid of a laser beam and/or by self-ionization. This experiment was the first time that positrons have been focused. The plasma lens was produced by laser and beam ionization of a neutral nitrogen gas jet injected into the plasma chamber by a fast-pulsing nozzle. The beam size was measured using a carbon-fiber wire scanner system and confirmed by the forward scattered synchrotron radiation.

\section{Thompson Scattering with X-ray lasers}

Another area of activity for ILSA last year was the use of $x$-ray lasers in TS to serve as a diagnostic of solid-density plasmas. This application has great potential to probe matter under extreme conditions,

\section{X-ray Protein Crystallography and Compton Source Development}

In the area of x-ray sources and applications, ILSA researchers used Compton scattering as a source of tunable x-rays for application to protein crystallography. The central theme of the research program is the detailed theoretical and experimental study of the interaction of relativistic electrons and coherent photon fields in vacuum. Electrons, the lightest of the charged leptons, are point-like particles without any internal structure. Photons are the quanta of the electromagnetic field. A good example of a coherent photon field is a laser pulse. Its spectrum (color content) is well defined, and a simple mathematical expression encapsulates all its characteristics.

The field of research involving $x$-ray sources and the interaction with charged particles contains a wealth of exciting phenomena. For example, ultrahigh-intensity laser pulses can coherently accelerate electrons in vacuum by generating very high electric field gradients (that is, the electrons gain much energy - thousands of electronvolts - over a short distance as measured in microns). The same laser pulses can also be used in collision with the relativistic electrons to produce $x$-ray flashes by way of the Compton scattering mechanism.

One of the most exciting applications of this research is in x-ray protein crystallography. Together with recombinant DNA technology, x-ray protein crystallography establishes a new paradigm for drug design and synthesis. One of the primary purposes of this work is to establish a theoretical formalism capable of fully describing the 3-D nature of the interaction as well as the influence of the electron and laser beam phase-space 
topologies upon the x-ray spectral brightness. This is the first detailed analysis of its kind. The new radiation theorem demonstrated and used in this work is general in nature, and it may represent a useful contribution to the field of classical electrodynamics. The key characteristics of an $x$-ray source useful for protein crystallography are small size, low angular divergence, good transverse coherence, and high-average spectral brightness. In turn, these requirements determine the necessary electron and laser-beam qualities.

In 2002, ILSA will start a research program that will use a compact, highquality, integrated electron linac in conjunction with a tabletop terawatt laser to produce high-brightness $x$-ray flashes at a repetition rate of 10 hertz. This system will demonstrate proof-of-principle Compton x-ray generation, and it will also allow the detailed benchmarking of ILSA's 3-D x-ray code. Another important component of this program is system scalability to higher repetition rates.

\section{Warm Dense Matter}

ILSA is initiating both experimental and computational studies in the area of warm dense matter. The construction of a shortpulse, tunable x-ray laser source will be a watershed for plasma-based research and will extend the ability of plasma physicists to study the regime of warm dense matter. Two separate areas of investigation are the laser probing of near-solid density plasmas, and the laser-plasma spectroscopy of ions in plasmas.

Warm dense matter refers to that part of the density-temperature phase space where the standard theories of condensed matter physics and/or plasma statistical physics become invalid. Warm dense matter, therefore, defines an intermediate region of temperature and density between solids and plasmas. This is a regime found in planetary interiors, in cool dense stars, and in every plasma device that starts from a solid, such as laser-solid-matter-produced plasma, as well as all inertial fusion schemes. In this state of matter, atomic, molecular, and ionic interactions may all be important. The properties of this state of matter are important to astrophysics and relevant to the production of inertially confined fusion reactions.

Previously, the study of dense plasmas has been severely hampered by the unavailability of laser-based methods. The single most useful diagnostic of local plasma conditions - the temperature $\left(T_{e}\right)$, the density $\left(n_{e}\right)$, and the ionization $(Z)-$ has been Thomson scattering. However, because visible light will not propagate at electron densities, $\geq 10^{22} \mathrm{~cm}^{-3}$ indicates that dense plasmas cannot be probed. The fourth-generation free-electron-laser(FEL-) based sources, such as the Linac Coherent Light Source (LCLS) and TESLA, will remove these restrictions. In other words, these FELs will do for plasma research what the visible laser did for physics starting in the late 1960s.

Laser-based plasma spectroscopic techniques have been used with great success to determine the line shapes of atomic transitions in plasmas, study the population kinetics of atomic systems embedded in plasmas, and look at the redistribution of radiation. However, the possibilities end for plasmas with $\mathrm{n}_{\mathrm{e}} \geq 10^{22} \mathrm{~cm}^{-3}$ because light propagation through the medium is severely altered by the plasma. Therefore, the development of the shortwavelength FELs will make available the entire field of high-Z plasma kinetics of importance to beam machines, laserproduced plasmas, astrophysical studies, and inertial fusion-related science. 


\section{Integrated Optical Waveguide Devices}

In the area of integrated optical waveguide devices, ILSA is exploring new ways of making integrated waveguide devices in glass. An example of such a device is the integrated frequencydoubled waveguide laser, schematically shown below in Fig. 49.

This device combines a waveguide laseremitting light at some frequency omega $(\omega-)$ with a frequency-doubling element, which converts the laser light to

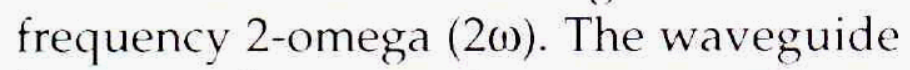
laser uses Bragg gratings as its cavity mirrors, and the $\chi^{(2)}$ grating is used to double the frequency of the fundamental laser beam. The successful fabrication of this kind of device requires the proper

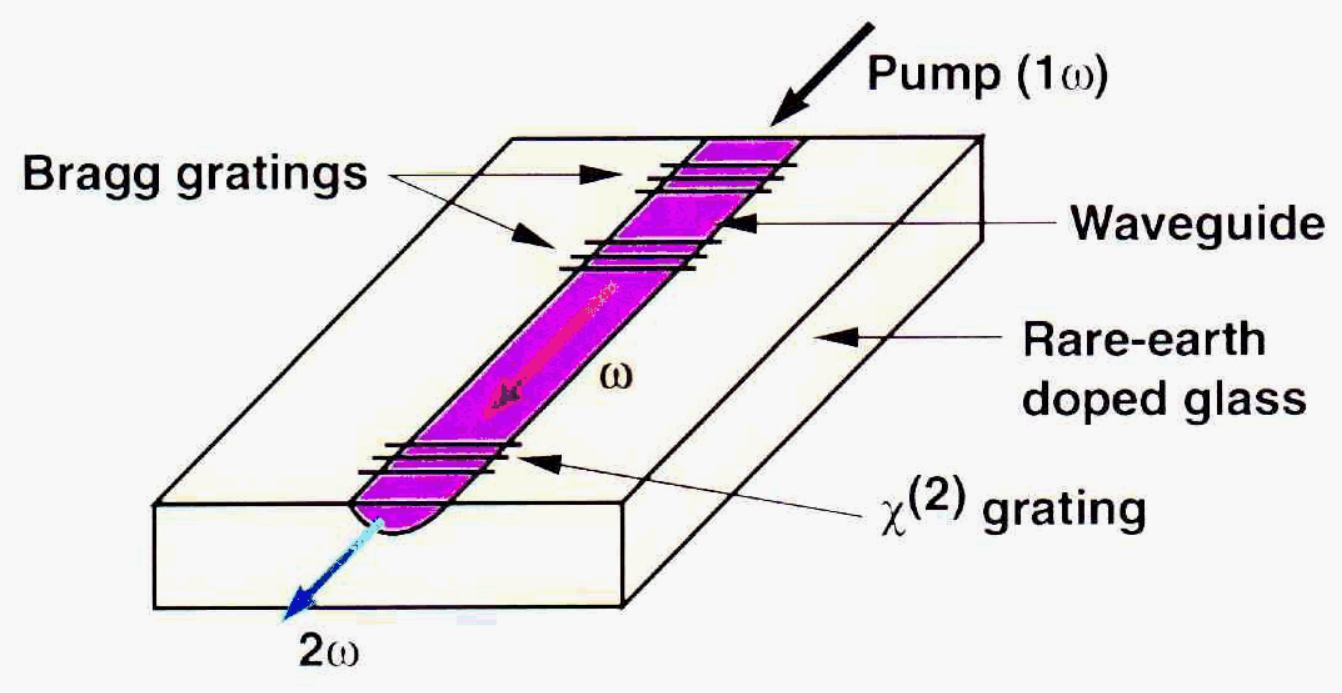

Fig. 49. This schematic diagram shows an integrated frequency-doubled waveguide laser under investigation by ILSA in the PAT Directorate. choice of host material and the subsequent addition of new functionalities to the glass through a number of different processing techniques.

The first required processing step is to fabricate a waveguide in the host glass through an ion-exchange process or the femtosecond-laser writing technique. In this latter technique, refractive index changes are induced inside a glass by using femtosecond-laser pulses tightly focused inside the material. Waveguides can be fabricated inside the glass by scanning the glass with respect to the focal point of the laser beam.

ILSA researchers are studying the properties of waveguides that are fabricated using this technique. Their investigation is focusing on the magnitude of the induced index change as well as the mode structure and optical loss of the waveguide. Researchers are also interested in understanding the atomic-scale structural changes that occur in the glass as a result of femtosecond-laser modification.

Recently, waveguides have been fabricated in fused silica using tightly focused, 800-nanometer, 130-femtosecond laser pulses at fluences between 5 and 200 joules per square centimeter. Other more complex, 3-D structures have also been fabricated, including curved waveguides, splitters, and interferometers. 


\section{PAT Awards 2000-2001}

\section{Fellowships}

\section{American Physical Society}

The American Physical Society (APS)

Fellowship recognizes investigators who have made advances in knowledge through original research or who have made significant, innovative contributions in the application of physics to science and technology. Each year, no more than one-half of 1 percent of the current APS membership is elected to the status of Fellow. During 2000 and 2001, four physicists from LLNL's Physics and Advanced Technologies (PAT) Directorate were named APS Fellows for their outstanding contributions to physics.

Physicist Ann Orel Woodin was elected a Fellow of the APS in November 2000. Woodin was honored for "pioneering the understanding and development of theoretical methods for studying excitation, ionization, and dissociation of polyatomic molecules." Woodin divides her time between the Laboratory and the University of California at Davis, where she is a professor in the Department of Applied Sciences and a graduate advisor. Her research is currently focused on what happens to low-energy electrons when they interact with molecules.

Robert Cauble, the PAT Condensed Matter Physics Division (H Division) leader, was also named an APS Fellow in November 2000. Cauble was cited by the APS for his "important contributions to the understanding of the equation of state (EOS) of dense, strongly coupled plasmas." Cauble's work included shocking matter to a million atmospheres' pressure to measure the EOS-how pressure varies with temperature and density —of that matter.
Peter Beiersdorfer, a Laboratory physicist in the High-Energy-Density Physics and Astrophysics Division, was elected to the status of APS Fellow in December 2001. Beiersdorfer, the group leader for Atomic Spectroscopy, was honored for his "many contributions to precision $\mathrm{x}$-ray spectroscopy of highly charged systems and application of this spectroscopy to plasma and astrophysical problems." Beiersdorfer, who joined the Laboratory in 1988, earned his bachelor's and master's degrees in physics at Auburn University, and his master's degree and Ph.D. in plasma physics at Princeton University.

Karl van Bibber, chief scientist in the PAT Directorate, was also named an APS Fellow in December 2001. Van Bibber was cited for his "leadership role in an ultrasensitive search for dark-matter axions and the conception of other elegant experiments for detection of the axion." Van Bibber received his bachelor's degree and Ph.D. at the Massachusetts Institute of Technology. He came to the Laboratory in 1985 from Stanford University, where he had been an assistant professor of physics. He started the High-Energy Physics and the Accelerator Technology Groups in 1991 and was the Laboratory's project leader for the construction of the B Factory.

\section{American Association for the Advancement of Science and Fusion Power Associates Leadership Award The American Association for the Advancement of Science (AAAS) Fellowship recognizes scientists for their efforts toward advancing science or fostering applications that are deemed scientifically or socially distinguished. In December 2000, LLNL physicist}

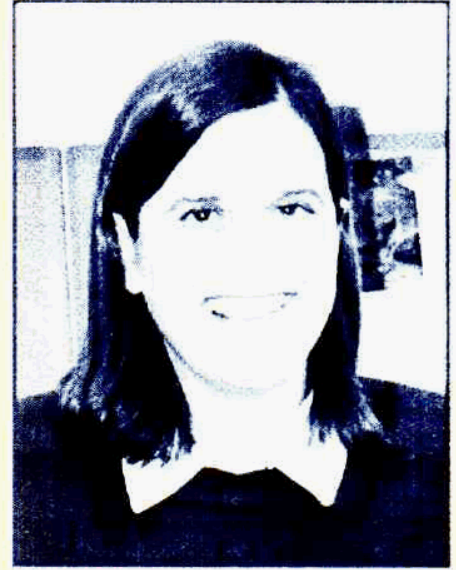

Ann Orel Woodin

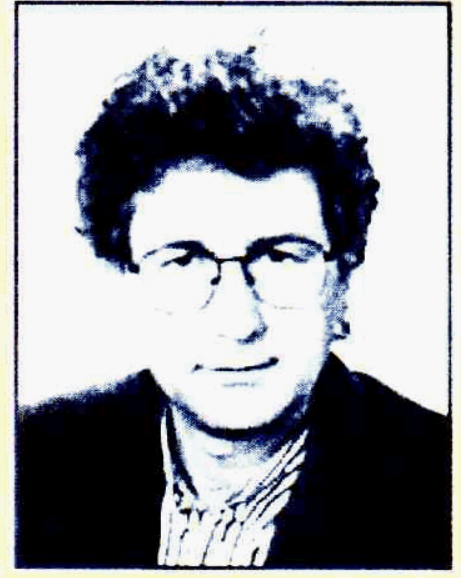

Robert Cauble

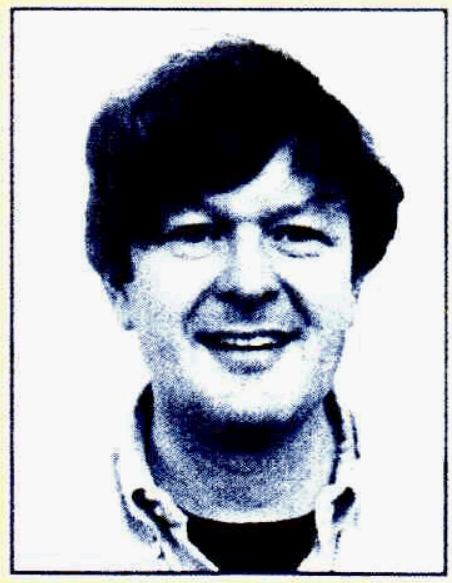

Peter Beiersdorfer

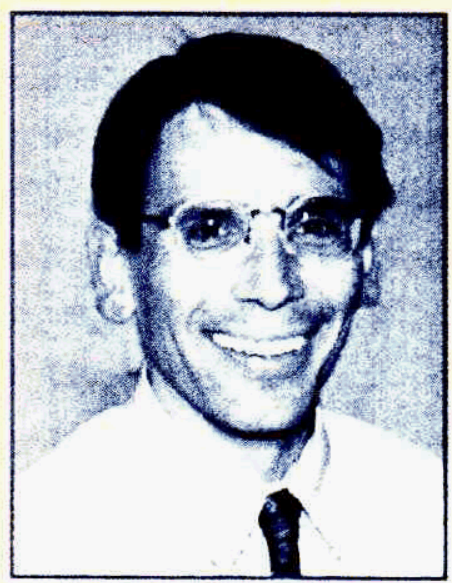

Karl van Bibber 


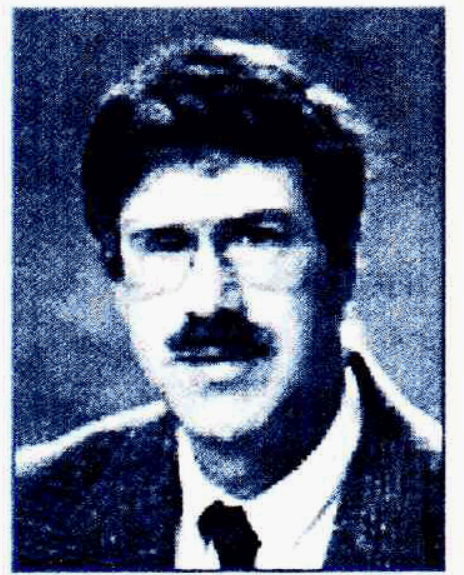

John Lindl

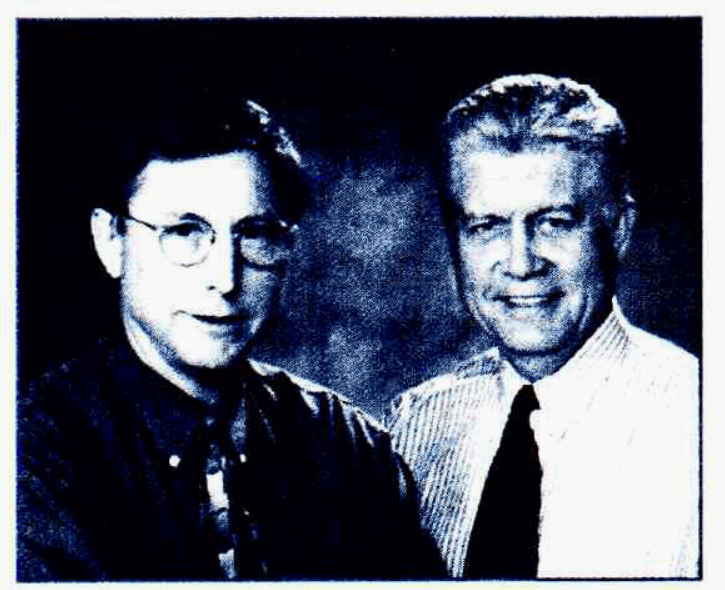

Lloyd Hackel and Ralph Jacobs
John Lindl was named an AAAS Fellow. Lindl was cited for his "distinguishing contributions to the understanding of high-energy-density matter and to the development of inertial confinement fusion."

In 2000, Lindl also received a Leadership Award from the Fusion Power Associates for his outstanding leadership qualities in accelerating the development of fusion. The Fusion Power Associates is a research and educational foundation that provides information on the status of fusion development and other applications of plasma science and fusion research.

Lindl, the head of the PAT Directorate's Fusion Energy Program, first joined the Laboratory in 1972 as a physicist in A Division-Laser Target Design. He served as associate program leader for theory and design in the Inertial Confinement Fusion (ICF) Program, division leader for $X$ Division-ICF Target Design, and acting ICF program leader before becoming the ICF Program's scientific director in 1994.

Lindl earned his bachelor's degree in engineering physics at Cornell University, and his master's degree and Ph.D. in astrophysics at Princeton University. Lindl was elected as an APS Fellow in 1984. Some of his other honors include the 1993 Edward Teller Medal in Inertial Fusion and the 1994 E. O. Lawrence Award. In 1998, Springer-Verlag published a book written by Lindl on the physics of inertial confinement fusion.

\section{Other Awards}

\section{Federal Laboratory Consortium for Technology Transfer-Excellence in Technology Transfer Award}

The Federal Laboratory Consortium (FLC) in Technology Transfer recognizes individuals and teams at federal laboratories with Excellence for Technology Transfer Awards for uncommon creativity and initiative in transferring to the private sector an advanced technology that significantly benefits industry, state and local governments, and/or the general public. Nominations are judged by representatives from industry, state and local governments, academia, and the federal laboratories.

An FLC Excellence in Technology Transfer Award was presented to physicist Steve Lane, associate program leader in the PAT Directorate's Medical Technology Program (MTP), and Kevin O'Brien, Connie Pitcock, Tom Peyser, Chris Darrow, and Joe Satcher. The MTP team has been working for more than five years on glucose monitoring technology that would be integrated with an insulin delivery device to form a biomechanical pancreas. Through MTP's work and in partnership with MiniMed, Inc., of Northridge, California, the biomechanical pancreas is being developed for both Types-I and II diabetes patients. LLNL's design encompasses a sensor that would be embedded under the skin of patients and that would monitor glucose levels and signal an insulin pump to administer insulin when needed. The technology is featured in the section on the MTP.

Lloyd Hackel and Ralph Jacobs of the NIF Programs and the PAT Directorates, respectively, received an FLC Award for their submittal, "LaserShot Peening System." Two other recipients were John Woolridge from the Office of Legal Counsel and Curt Thiesen from the Industrial Partnerships and Collaboration Office. This technology is designed to extend the life of critical metal partsfrom aircraft engine fan blades to artificial knee and hip joints. The technique involves repeatedly striking materials with intense shock waves generated by a pulsed laser beam, thereby inducing compressive stress that reduces metal failure and corrosion cracking. LaserShot peening will augment conventional shot peening, in which materials are bombarded with very tiny metal balls. The LaserShot Peening technique will be used in areas where greater depths of compressive stress are required to keep high-value parts, such as jet engines, from failing. The LaserShot Peening System is the subject of a cooperative research and development agreement between LLNL and Metal Improvement Co., Inc., (MIC) of Paramus, New Jersey. 
An FLC Award was given to the LLNL team that included PAT researchers for "PEREGRINE, a 3-D Monte Carlo Radiation Dose Calculation Therapy System," a revolutionary tool for analyzing and planning radiation treatment for cancer patients. PEREGRINE combines the Laboratory's expertise in nuclear physics with its proficiency in computer modeling to deliver a system that more exactly estimates the radiation dose being delivered to a specific tumor and nearby tissue. Led by the principal investigator/program leader, PAT's Christine HartmannSiantar, the PEREGRINE team includes Brian Guidry, Don Jong, Rosemary Walling, Ed Moses, Tom Daly, Sarita May, Paul Bergstrom, Ralph Patterson, Don Fujino, Ron House, Jim Rathkopf, Clark Powell, Dave Knapp, Larry Cox, Lila Chase, Dewey Garrett, Steve Hornstein, Bill Chandler, and Alexis Schach von Wittenau. Also named in the award were Jim Sommercorn of the Industrial Partnerships and Commercialization Office, and John Woolridge of the Laboratory Counsel's Office. The PEREGRINE technology has been licensed to NOMOS Corporation of Sewickley, Pennsylvania.

\section{U.S. Department of Energy Bright Light Award}

The Bright Light Award honors discoveries and innovations from the DOE complex that benefit the American public, contribute to U.S. competitiveness in the global marketplace, and have the potential for significant growth. A panel of DOE citizen judges, who had to choose five innovations from among 23 discoveries or innovations developed between 1999 and 2000, selected the recipients of the Bright Light Awards.

The MTP-led team was one of the research groups honored nationwide for its work on a recent consumer-oriented innovation. The team members have been working for more than five years on the glucose monitoring technology that will be integrated with an insulin delivery device developed by MiniMed, Inc. Led by physicist Stephen Lane of MTP, the LLNL team is shown in

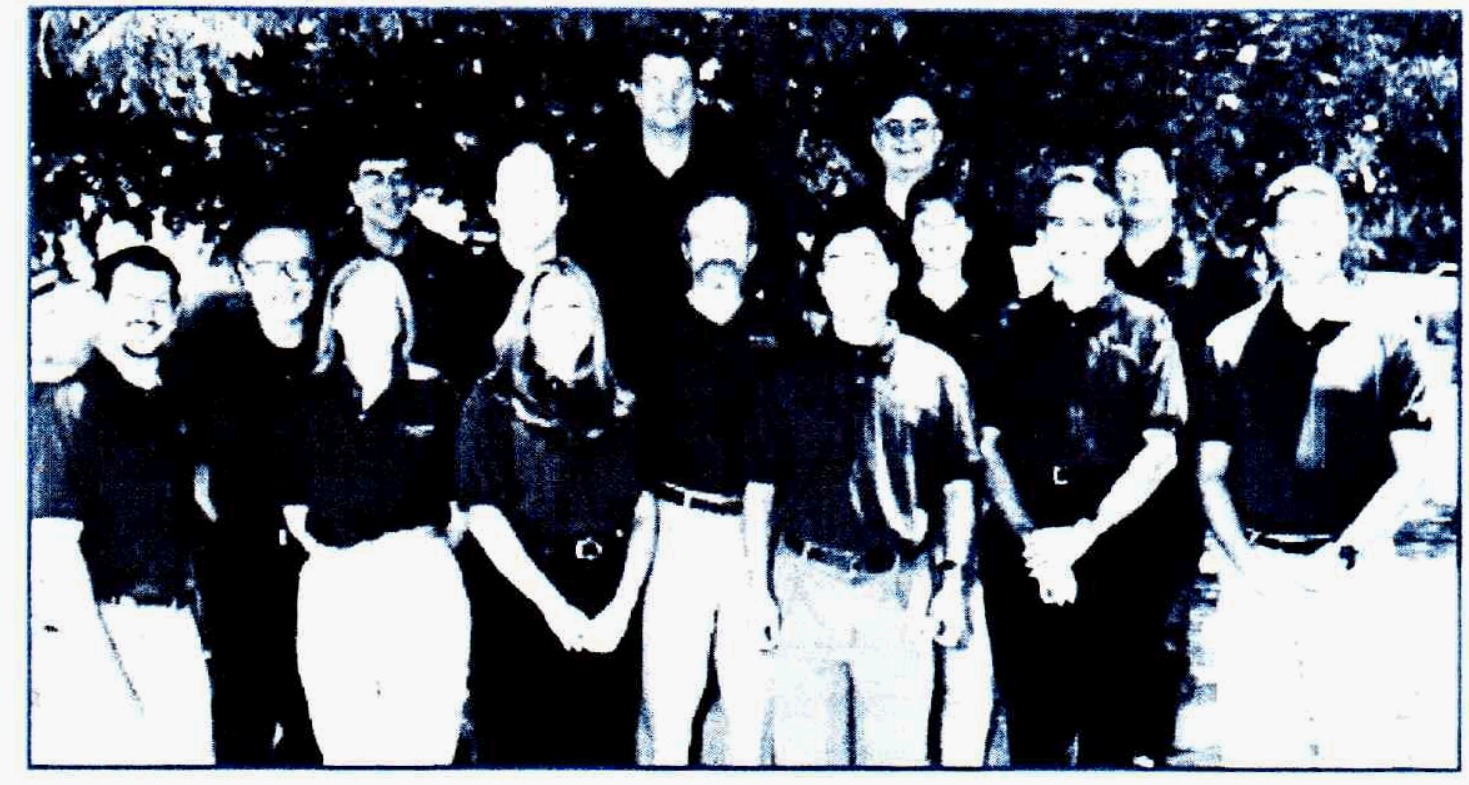

Members of the PEREGRINE team are (left to right): Brian Guidry, Don Jong, Rosemary Walling, Ed Moses, Tom Daly, Sarita May, Paul Bergstrom, Ralph Patterson, Don Fujino, Ron House, Christine Hartmann-Siantar, Jim Rathkopf, Clark Powell, and Dave Knapp. Not pictured are Larry Cox, Lila Chase, Dewey Garrett, Steve Hornstein, Bill Chandler, and Alexis Schach von Wittenau.

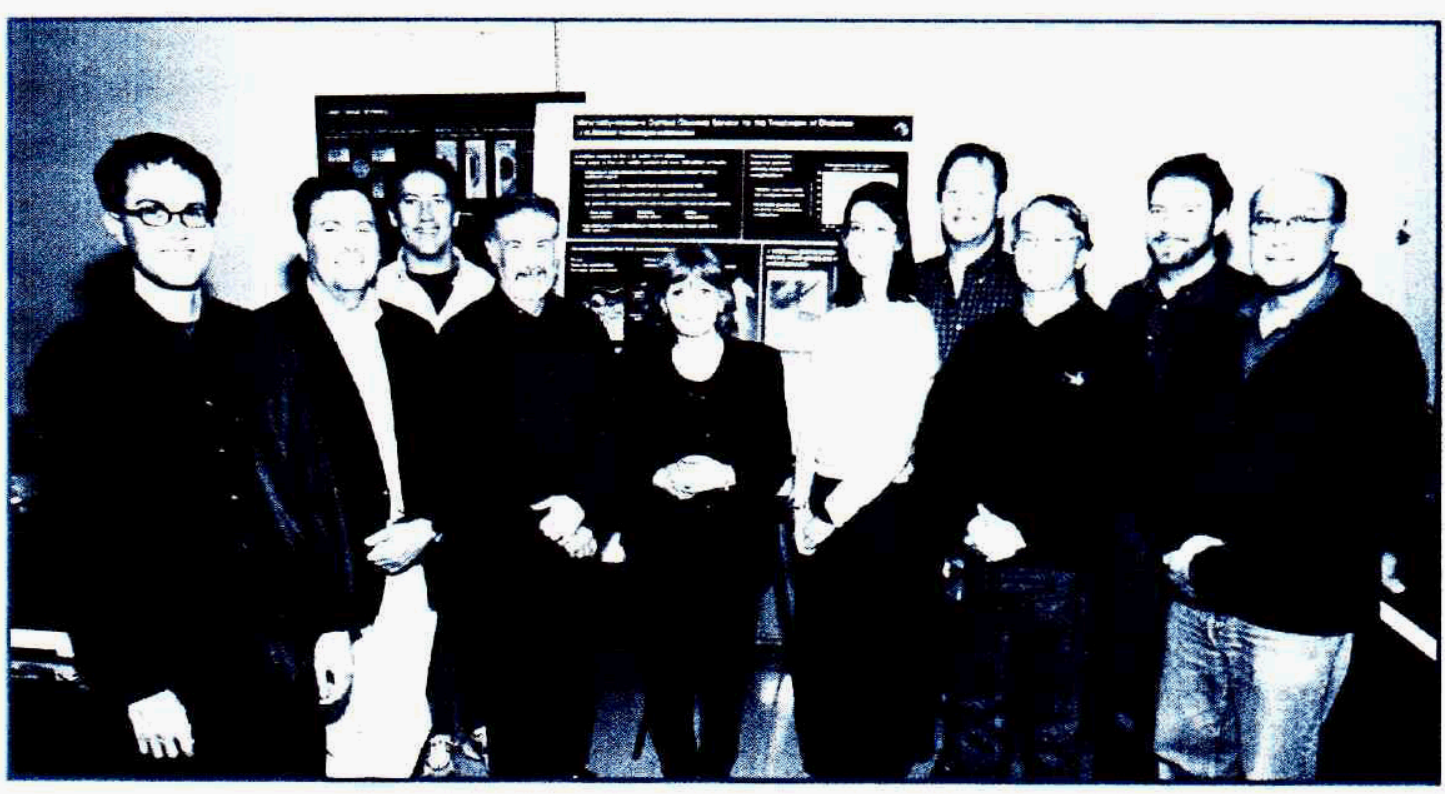

The DOE Bright Light winners, from left:Doug Cary, Tom Peyser, Robert Sanner, Steve Lane, Natasha Zaitseva, Anne Escaron, Robert Glass, Joe Satcher, Robert Maxwell, and Bob Reibold. Not pictured are Glenn Fox, Chris Darrow, and Karen Lauer. This team also received a FLC Excellence in Technology Transfer Award.

the photo above. A Bright Light Award was presented to the research team at a White House ceremony in January 2001.

\section{American Astronomical Society- Beatrice M. Tinsley Prize}

In 2000, the American Astronomical Society awarded the Beatrice M. Tinsley Prize to Charles Alcock, former head of PAT's Institute for Geophysics and Planetary Physics. The Beatrice Tinsley Prize recognizes outstanding research contributions to astronomy or astrophysics of an exceptionally creative or innovative character. The LLNL-led international project team tested the hypothesis that a significant fraction of the dark

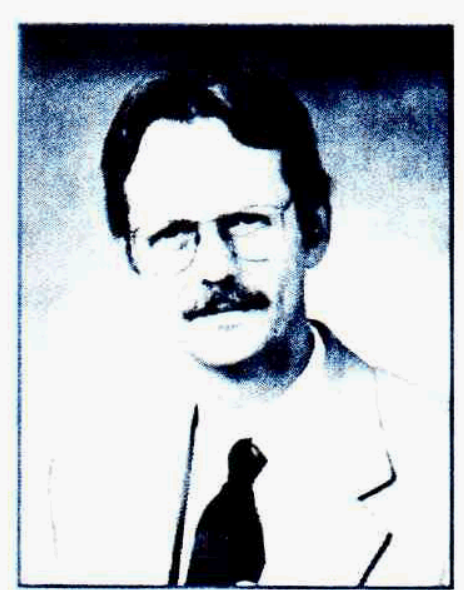

Charles Alcock 


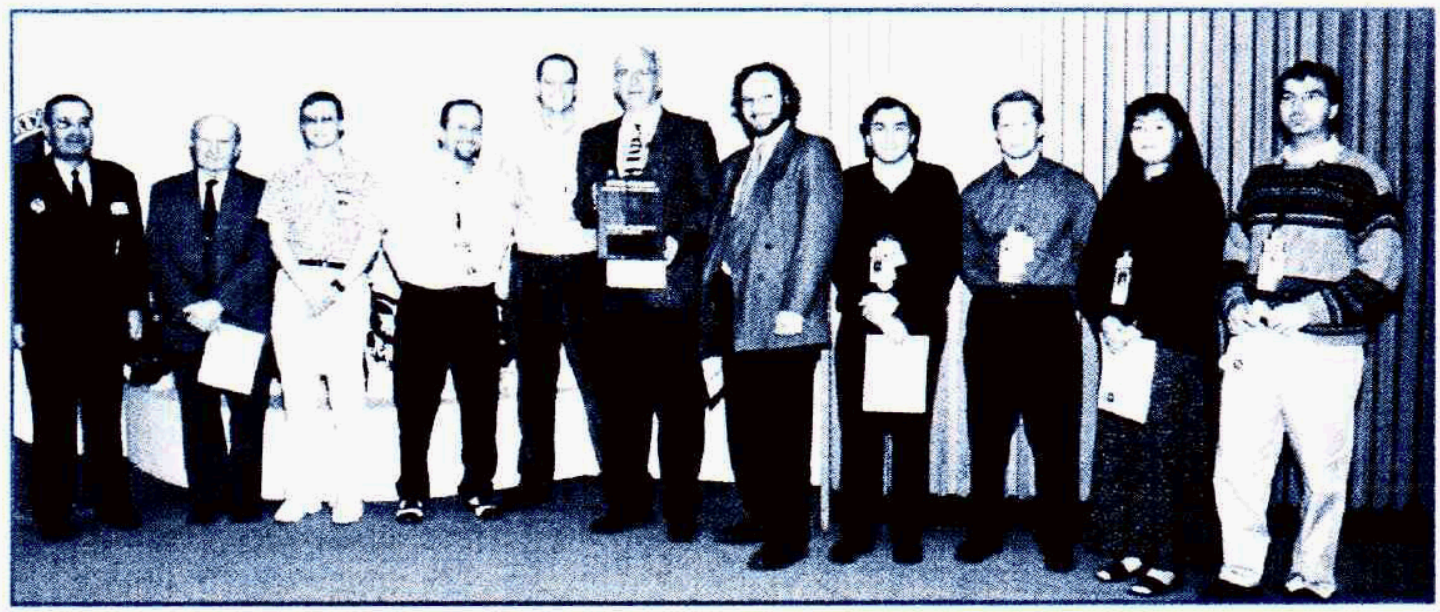

Pictured with Brig. Gen. Thomas Giaconda (left) are members of the GEANIE Cross Section Measurement team, from left: Rudolf Bauer, Mark Stoyer, Daniel Archer, Dennis McNabb, John Becker, Lee Bernstein, Walid Younes, Paul Garrett, Hong Chen, and Petr Navratil. Not pictured are Christopher McGrath, W. Erich Ormand, John Anderson, and Frank Dietrich.

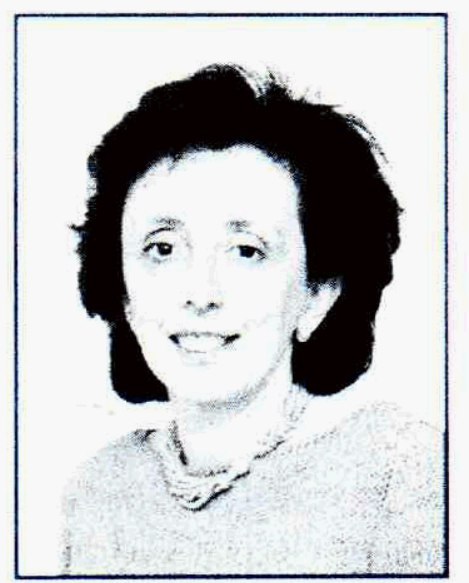

Giulia Galli

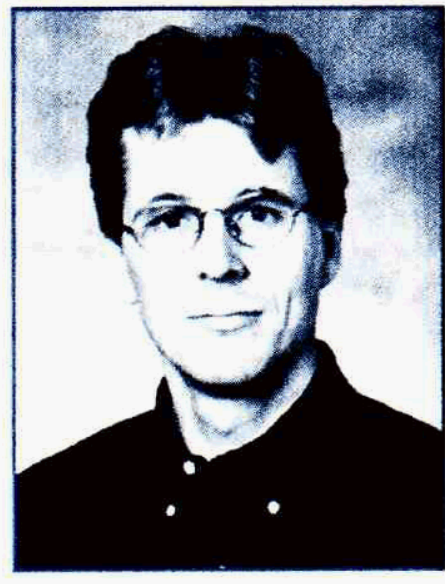

François Gygi

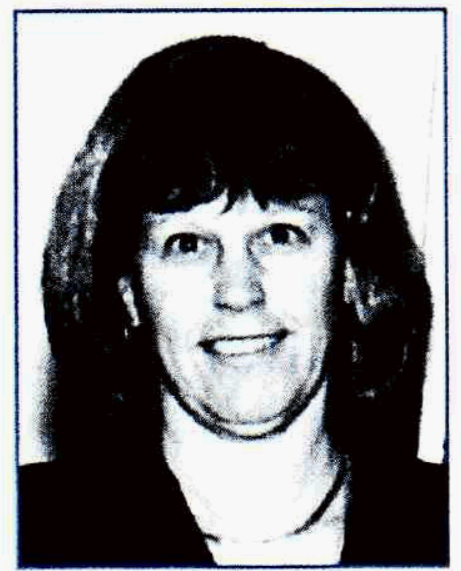

Ellen Corey matter in the halo of the Milky Way galaxy is made up of objects like brown dwarfs or planets, which have come to be known as Massive Compact Halo Objects or MACHOs.

\section{Weapons Recognition of Excellence Awards (1998-2000)}

The LLNL Defense and Nuclear Technologies Directorate, the National Nuclear Security Administration, and DOE handed out 17 awards for weapons excellence to 127 Laboratory employees in 2001. The Weapons Recognition of Excellence Awards are given annually to recognize people whose career achievements often go unnoticed, in part because of security reasons. Four teams from the PAT Directorate were honored with the Weapons Recognition of Excellence Awards.

One award was given to the GEANIE Cross Section Measurements Team at LLNL, which includes John Becker, Lee Bernstein, Walid Younes, Dennis McNabb, Paul Garrett, Daniel Archer, Christopher McGrath, Mark Stoyer, Hong Chen, W. Erich Ormand, Petr Navratil, John Anderson, Frank Dietrich, and Rudolf Bauer. This team collaborated with a team from Los Alamos National Laboratory (LANL) to achieve a definitive measurement of the production of plutonium-238 from neutron bombardment of plutonium-239 over a wide neutron energy range. This work was performed with the new Geranium Array for Neutron-Induced Excitations (GEANIE) at the Los Alamos Neutron Science Center-Weapons Neutron Research (LANSC/WNR) Facility neutron source. To establish the absolute cross section, the teams used a new analysis technique that combined measurements of known gamma-ray transitions from the product nucleus and state-of-the-art reaction modeling to calculate and correct for unobserved decays. This project has provided important data for the weapons program that were difficult, or even impossible, to obtain in other ways. The apparatus and techniques that were developed are being applied to other priority data needs for the Stockpile Stewardship Program.

Giulia Galli and François Gygi also received a Weapons Recognition of Excellence Award. Galli and Gygi have exploited Advanced Simulation and Computing (ASCI) computing to pioneer the development of a fundamental, new method of materials simulation. Their terascale calculations have demonstrated that first-principles quantum molecular dynamics simulations can provide profound insight into the behavior of shocked, high-pressure molecular fluids, which are relevant to the detonation and performance of high explosives and to the behavior of hydrogen.

Another award was given to the Livermore Equation of State (LEOS) team of Ellen Corey and David Young. Corey and Young have devoted many years to the development of EOS models and to the representation of these models in libraries of data tables and access routines. This effort came to fruition with the release of LEOS, a new EOS library containing data for more than 150 materials in denser tables with greatly improved interpolation 
routines. The timely release of these tables and collaborative efforts among the awardees and developers of an ASCI burn code were essential to the successful demonstration of the 3-D, full-system simulation prototype.

A Weapons Recognition of Excellence Award was also awarded to the Proton Radiography Advanced Technology Team, which includes Peter Barnes, Anthony Chargin, Edward Hartouni, Jeff Hockman, Mike Kreisler, Lloyd Multhauf, and Ron Soltz. This team led the LLNL effort to investigate the use of high-energy protons as a probe for weapons-related dynamic radiography. Using facilities at LLNL and LANL, the team developed and executed a suite of critical experiments that proved protons could be used to measure the energy lost traversing a thick target, enabling information to be extracted about target density. This required not only careful experimentation but also the design and implementation of an innovative detector. An additional series of experiments at Brookhaven National Laboratory produced more than 3,000 detailed, highresolution radiographs that have been used to answer many questions about the utility of this new radiographic probe.

\section{Edward Teller Fellowships}

The Edward Teller Fellowships recognize and encourage scientific accomplishments and provide Fellows with the flexibility to explore new areas of interest by allowing each recipient to do self-directed work for the Laboratory over the next year. The Fellowships have been awarded for two consecutive years, 2000 and 2001. In each year of the Fellowship's existence, scientists from the PAT Directorate have received two of the four Fellowships.

Bill Nellis was one of the recipients of the 2000 Edward Teller Fellowship. Nellis, who discovered a method for achieving metallized fluid hydrogen, was recognized for his "contributions of long-standing

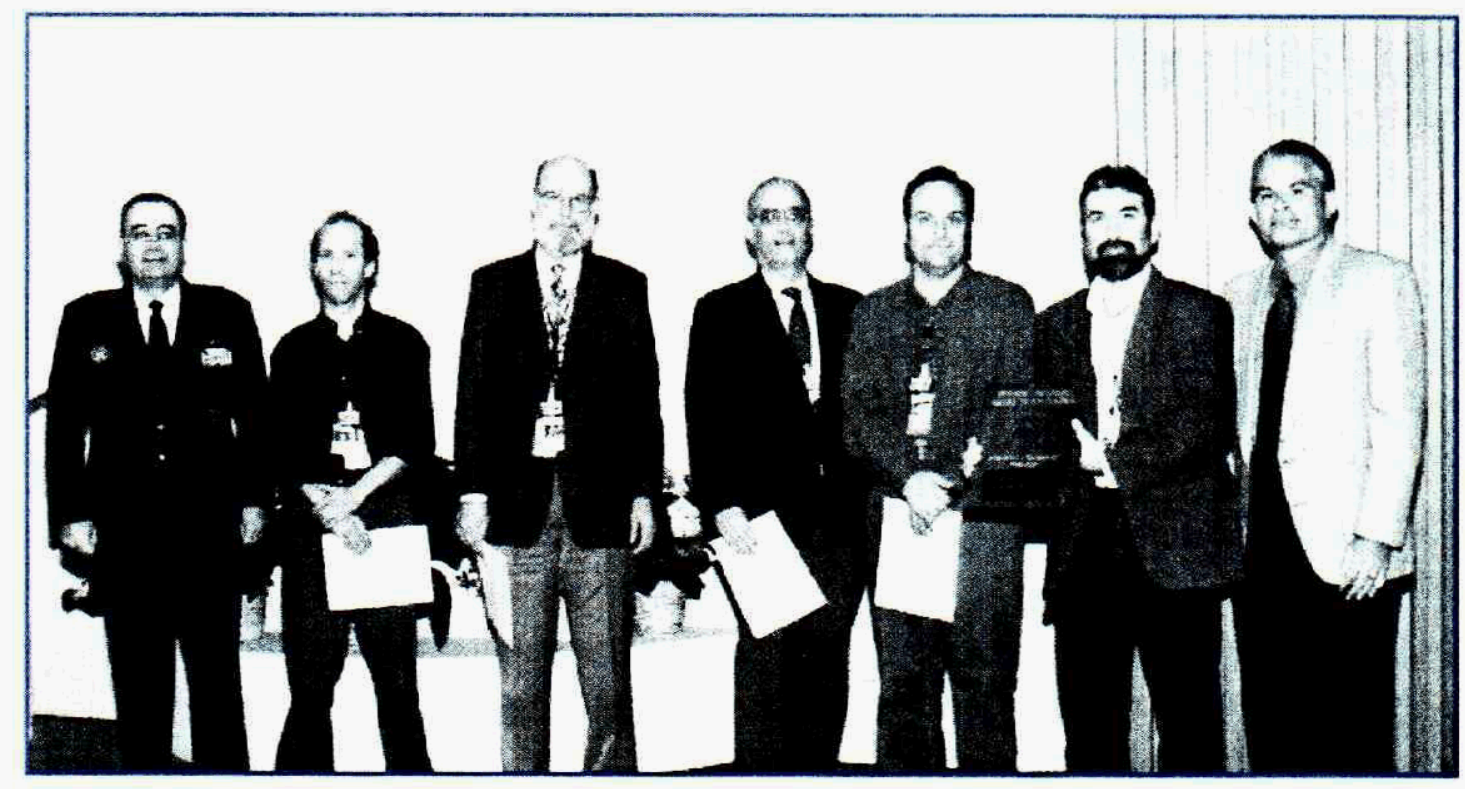

Pictured with Brig. Gen. Thomas Gioconda (at left) are members of the Proton Radiography Advanced Technology Team, from left: Ron Soltz, Lloyd Multhauf, Mike Kreisler, Peter Barnes, Ed Hartouni, and Jeff Hockman. Not pictured is Anthony Chargin

scientific excellence and impact in and on the field of shock physics." Most noteworthy, according to his award, are his "innovative research efforts in the use of impact-generated shocks to measure the properties of dense, warm molecular and atomic fluids." The Edward Teller Fellowship enabled Nellis to work as a visiting Fellow at Trinity College of Oxford University and to start a book on fluids at high pressures and temperatures.

A second recipient of the 2000 Edward Teller Fellowship was Christine Hartmann-Siantar, the PAT principal investigator for the PEREGRINE program. PEREGRINE uses Monte Carlo computer methods to help control x-ray cancer treatments. Hartmann-Siantar was honored for her "unique and exemplary record of important scientific discoveries and leadership in the PEREGRINE program." Hartmann-Siantar, who holds a Ph.D. in medical physics from the University of Wisconsin, joined the Laboratory in 1993. She has received many honors, including the DOE Young Independent Scientist Award in 1996, the Presidential Early Career Award for Scientists and Engineers in 1996, and an R\&D 100 Award in 1999. Hartmann-

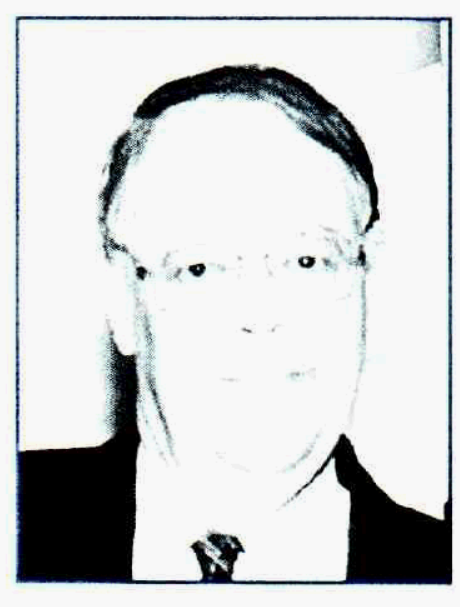

David Young

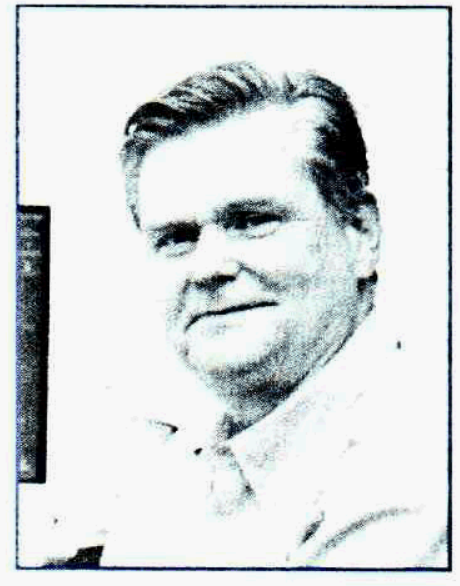

Bill Nellis

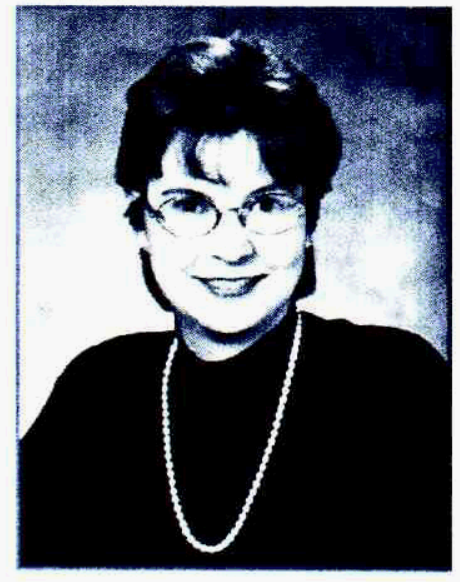

Christine Hartmann-Siantar 


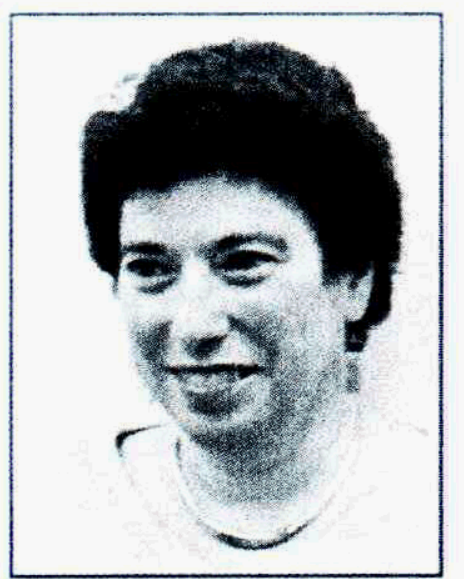

Claire Max

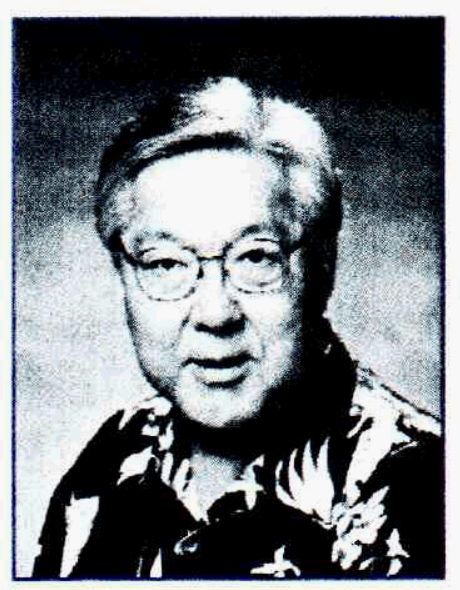

George Kwei

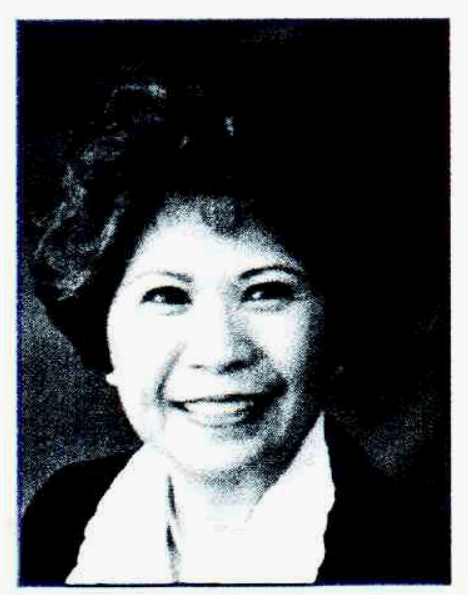

Chung Bothwell
Siantar used her Edward Teller Fellowship to study how radiation damages DNA.

Claire Max, a recipient of the Edward Teller Fellowship in 2001, heads the Laboratory's Laser Guide Star Project. Central to this project are adaptive optics systems that improve the resolution of ground-based astronomical telescopes. Max was also instrumental in creating the new Center for Adaptive Optics, which is headquartered at the University of California at Santa Cruz. The Teller Fellowship enabled her to devote herself to a program of intensive, cutting-edge exploration in adaptive optics and its emerging applications. Max also planned to continue her mentoring of young researchers supporting this work and to define a strong role for LLNL within the Center for Adaptive Optics and related communities.

Physicist George Kwei also received a 2001 Edward Teller Fellowship. Kwei is a leader in neutron-scattering research relevant to the Stockpile Stewardship Program. He planned to use his Fellowship to write a book on science policy in the White House. His proposed book would address how policy advisers work with the President and Congress to set policy. Kwei would like to use this book to explain to readers in the general public how they benefit from science.

\section{Lybrand Certificate of Merit}

Chung Bothwell, Resource Manger for PAT's Fusion Energy Program at LLNL and director for the UNCLE Credit Union since 1994, received a Lybrand Certificate of Merit in June 2001 in the annual manuscript competition sponsored by Strategic Finance Magazine. Bothwell's manuscript, "How a Small Credit Union Skyrocketed to More than \$100 Million in Assets," described the UNCLE Credit Union's growth over a 20-year span and the strategic steps taken to achieve this growth.

\section{Recognition for PAT Research \& Development}

\section{Laboratory's 2000 Physics Research among the Best}

Three PAT-led research projects at the Laboratory were noted among the top physics breakthroughs, according to Physics News in 2000. The publication is the most recent in a series of annual surveys of important physics stories worldwide prepared by the American Institute of Physics (AIP). It includes the most important discoveries of the year 2000 in the fields of astrophysics; atomic, molecular, and optical physics; biologica! and medical physics; condensed matter and materials physics; particle and nuclear physics; and plasma physics.

Among the important physics stories included in Physics Newes in 2000) are the following: (1) the metallization of liquid molecular deuterium performed on the Nova laser at LLNL, led by Peter Celliers; (2) the demonstration of a plasma lens for gigaelectron-volt electrons and positrons at LLNL, led by Hector Baldis; and (3) high-performance, first-principles simulations of collisionally ionized hydrogenthe simplest, nontrivial, three-body Coulomb scattering problem in quantum mechanics-performed as a collaboration between LLNL and LBNL, led by Tom Rescigno.

\section{Extreme Ultraviolet Lithography Named Technology of the Year}

MicroDesign Resources' Microprocessor Report, a respected computer industry analysis newsletter, has named Extreme Ultraviolet Lithography (EUVL) its "Technology of the Year" in 2001. The award recognizes the importance of EUVL technology to the rapidly increasing speed at which supercomputers can process increasingly large amounts of data.

According to Don Sweeney, leader of L.L.NL's participation in the Virtual National Laboratory (VNL), "This award is very important within the industry. There is only one given per year." VNL is 
the consortium of LLNL, Law rence Berkeley National Laboratory (LBNL), and Sandia National Laboratories (SNL) that developed this next-generation method of microchip lithography.

Funding for developing EUVL technology has come from the private sector. Intel, Advanced Micro Devices, Motorola, Micron Technology, and Germany's Infineon Techologie AG created the limited liability company, EUV LLC, a cooperative research and development agreement, to back the project. IBM joined the consortium in March 2001. Other integratedcircuit manufacturing companies, whose markets depend on emerging technologies, are expected to add their support.

\section{PAT Awarded Two of LLNL's Eight University of California Campus-Laboratory Collaborative Projects and Exchanges}

Laboratory researchers have been awarded eight of the 11 research projects and exchanges that will be carried out collaboratively among the laboratories and the University of California (UC) campuses. The projects and exchanges were chosen competitively and will be funded at a level of $\$ 1.5$ million total per year. Researchers in the PAT Directorate are collaborating with researchers from UC campuses in two of the selected projects.

PAT researcher Stavros Demos will work with Robert Brasch from UC San Francisco to develop noninvasive techniques for the diagnosis of breast cancer using optical lasers. The project will be funded with $\$ 270,000$ during the first year and $\$ 160,000$ during the second year.

In a separate project, PAT scientist William Craig will work with S. E. Boggs from UC Berkeley to develop new capabilities in medical imaging, using gamma-ray detectors that were originally developed for astronomy. This project will be funded with $\$ 240,000$ during the first year and $\$ 160,000$ during the following year.

\section{Additional Noteworthy Items from PAT}

\section{"Smart Probe" Development and Testing}

In January 2001, the PAT-developed

"Smart Probe," a new minimally invasive diagnostic tool in the fight against breast cancer, became the subject of a cooperative research and development agreement with BioLuminate, of San Jose, California. In October 2001, BioLuminate announced it would begin testing the new diagnostic on human volunteer patients at the UC Davis Medical Center.

\section{Massive Compact Halo Objects Collaboration Findings}

In January 2001, Laboratory researchers with the Massive Compact Halo Objects (MACHO) collaboration, a project of DOE and the National Science Foundation, presented findings of strong evidence that microlensing events are caused by compact dark matter in the halo of the Milky Way. In a related finding, MACHO researchers reported finding for the first time 154 stars rapidly moving toward the center of the galaxy. In September 2001, the MACHO project yielded a treasure trove of data on 73 million stars. In December 2001, PAT astronomers, in collaboration with an international team of researchers, made the first detection and measurement of the properties of a dark-matter object in the Milky Way. The observation of a gravitational microlensing event - a temporary increase in the brightness of a background star during the time it takes dark matter to pass in front of the star-was detailed in the December 6, 2001, issue of Nature. 


\section{Guide Star Installed at Keck Observatory}

PAT scientists played key roles in creating a "virtual" guide star over Hawaii. The virtual guide star will be used with adaptive optics on the Keck II telescope to greatly increase the resolution of fine details of astronomical objects. For more than two years, Deanna Pennington, Curtis Brown, Pam Danforth, and Holger Jones worked on the Keck telescope, redesigning, demonstrating, and commissioning the laser system on the telescope so that the virtual guide star could achieve "first light." Claire Max, principal investigator on the project, was honored with a Teller Fellowship for her contributions. Adaptive optics is featured in the section on the Laser Science and Technology Program.

\section{New Defense Advanced Research Projects Agency Initiative}

In June 2001, under a new Defense Advanced Research Projects Agency (DARPA) initiative, PAT teamed with academic institutions and industry to develop powerful new capabilities for (1) multigigabits-per-second, secure, freespace communication links, and (2) aberration-free, 3-D imaging and targeting at ranges longer than 1,000 kilometers.

\section{Publication of First Results from the B Factory}

In June 2001, the search to resolve a longstanding mystery of particle physics reached a milestone with the publication of the first results from the B Factory experiment at the Stanford Linear Accelerator Center (SLAC). PAT scientists played a key role in analyzing the data from the experiment, which was designed to resolve the mystery of why there is more matter than antimatter in the universe. The B Factory project was honored with a DOE Program and Project Management Award in November 2000.

\section{Use of Superconducting Tunnel Junction Detectors Featured in Synchrotron Radiation News}

The high-spectral resolution of the superconducting tunnel junction detectors provides a way to measure the weak fluorescence from transition-metal $\mathrm{L}$ lines that would otherwise be buried in the wings of much stronger neighboring lines. The results of this work generated much interest in the scientific community and were featured in the March/April 2001 issue of Synchrotron Radiation News as noted in the section on the Imaging and Advanced Detectors Division.

\section{New Director of the Glenn T. Seaborg Institute \\ In June 2001, Christine Hartmann- \\ Siantar, principal investigator of the PEREGRINE Program, was selected as director of the Glenn T. Seaborg Institute.}

\section{Unveiling of 10-Kilowatt Laser}

In September 2001, representatives from the NIF Programs and the PAT Directorates, along with U.S. Army officials, unveiled a 10-kilowatt, solidstate, heat-capacity laser that could demonstrate a new short-range air- and missile-defense capability.

\section{Three-Year Extension of EUVL}

In October 2001, the Laboratory's largest ever cooperative research and development agreement, centered in the PAT Directorate- to develop Extreme Ultraviolet Lithography — was extended for another three years. 


\section{PAT Directorate's Striving for Excellence}

The PAT Directorate strives for excellence. Its successes since its inception in 2000 are demonstrated, in part, by the following metrics.

\begin{tabular}{|c|c|c|}
\hline Category & $2000 *$ & 2001 \\
\hline $\begin{array}{l}\text { Major Awards } \\
\text { (e.g., E. O. Lawrence, Edward Teller, Fusion Power Associates, } \\
\text { APS, OSA, IEEE, ANS, Defense Weapons Award of Excellence) }\end{array}$ & 12 & 10 \\
\hline R\&D 100 Awards and Federal Laboratory Consortium Award & 3 & 1 \\
\hline Society Fellowships & 2 & 3 \\
\hline Patent Disclosures & 28 & 11 \\
\hline Patent Applications & 24 & 12 \\
\hline Patents Issued & 20 & 19 \\
\hline Newly Executed Licenses & 4 & 5 \\
\hline Total Licenses & 40 & 38 \\
\hline Licensing Income & $\$ 325 k$ & $\$ 669 k$ \\
\hline Journal Publications & 185 & 193 \\
\hline Conference Presentations and Proceedings & 364 & 427 \\
\hline U.C. Science and Technology Assessment & Outstanding ${ }^{* *}$ & Outstanding ${ }^{* *}$ \\
\hline
\end{tabular}

* PAT Directorate formed on 7/1/2000. Totals include results for predecessor groups for first half of 2000.

${ }^{* *}$ Highest rating 


\section{Research Retrospectives: Fusion Ene}

Lawrence Livermore National Laboratory is celebrating its 50th Anniversary in 2002. These three retrospectives are presented by the PAT Directorate and its predecessor organizations to show the impact of physics activities at the Laboratory throughout its history.

\section{Highlights of Fusion Energy Research at LLNL}

Bick Hooper and John Lindl developed this timeline of achievements in fusion energy research at LLNL.

\section{0s}

- Originally known as Project Sherwood, the Magnetic Fusion Energy (MFE) program began in 1952 with the founding of the Laboratory. The MFE program pioneered magnetic fusion research with such devices as the Astron, Levitron, Table Top, Toy Top, and ALICE.

- With the Gibson-Lauer experiment, LLNL scientists verified how a particle moves in a magnetically confined plasma, thus demonstrating the feasibility of the magnetic "mirror" device.

- Project Sherwood was declassified, allowing researchers to publicly share ideas and achievements. Declassification allowed LLNL researchers to attend the Geneva "Atoms for Peace" Conference in 1958. This conference brought together fusion researchers from the United States, Europe, the Soviet Union, and Japan, and established a pattern of international collaboration that has continued and grown.

\section{0s}

- Fusion neutrons were produced in the Toy Top machine with pulsed fields greater than 20 tesla.

- Astron, LLNL's first large-scale magnetic fusion experiment, began operation. High-current induction accelerator technology invented for this project is used today in x-ray production for radiography, a millimeter-wave free-electron laser (FEL), and heavy-ion fusion research.

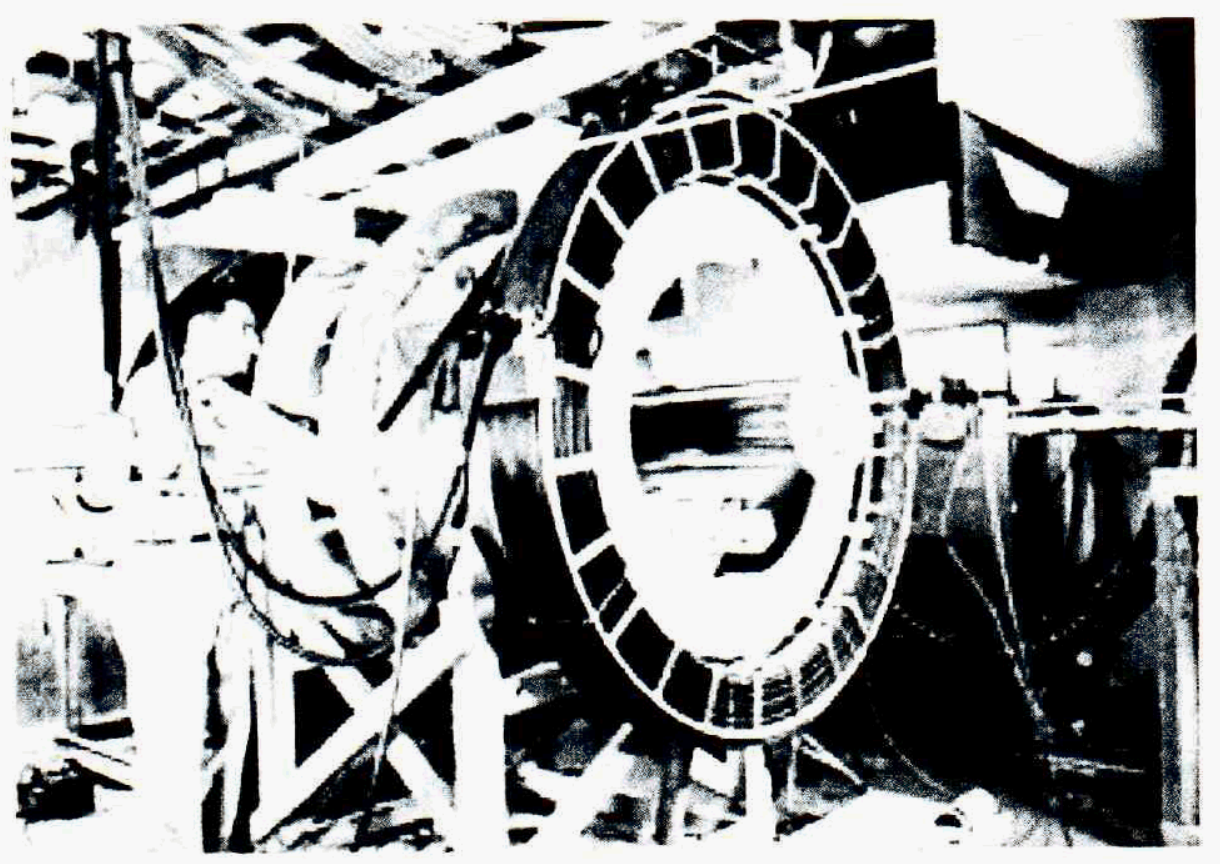

Part of Project Sherwood in the 1950s, Table Top was an early machine that LLNL researchers used to explore the use of magnetic mirrors to confine hot plasmas for fusion energy.
- Magnetohydrodynamic (MHD) plasma instabilities were successfully eliminated by containing the plasma in a magnetic well. LLNL researchers pioneered new magneticwell configurations, starting with ALICE and Baseball I, which used a magnet coil shaped like a baseball seam.

1970s

- The 2XII device was LLNL's first magnetic fusion machine to achieve densities and temperatures in the fusion reaction range.

- The Baseball II's superconducting magnet became operational. Baseball II was the first large superconducting magnet machine to be used in fusion research. Development of the magnet led to significant gains in superconducting technology.

- The Rotating Target Neutron Source Facility (RTNS-II) became operational. RTNS-II provided the world's only intense source for studying $14-\mathrm{MeV}$ neutrons and their effect on materials used in fusion reactors.

- The first users at the National Magnetic Fusion Energy Computer Center (NMFECC) logged on to LLNL's \$8-million G-machine. NMFECC later installed the world's first Cray-2 computer. Now known as the National Energy Research Supercomputer Center (NERSC), this facility serves fusion and other researchers nationwide.

- With the 2 XIIB machine, LLNL researchers achieved a stable plasma state at high temperature and density. By injecting neutral beams, they achieved beta (ratio of plasma pressure to magnetic field pressure) values of approximately 2 --still the most efficient use of a magnetic field to confine plasma.

- The first use of a tandem mirror to confine the fusion plasma occurred in the Tandem Mirror Experiment (TMX).

- The first in a series of heavy-ion-fusion workshops were organized (with Lawrence Berkeley Laboratory [LBL]) to explore the concept of heavy-ion-driven fusion and develop it in detail.

- The first liquid wall concept (using Li) was developed for a fusion reactor chamber, first for Astron and then a few years later in much more detail for inertial fusion energy (HYLIFE-I).

\section{0s}

- TMX-Upgrade tested the thermal barrier concept and improved the tandem mirror's ability to confine the plasma.

- Construction of the Mirror Fusion Test Facility-B (MFTF-B), a tandem mirror machine benefiting from the TMX-Upgrade experiments, was completed; and the MFTF-B successfully passed engineering testing.

- Construction began on the Microwave Tokamak Experiment (MTX). In collaboration with the Japan Atomic Energy Research Institute (JAERI), MTX investigated electron plasma heating and control in a tokamak device. Microwaves from a FEL or gyrotron heated the plasma.

- The United States, the European Community, Japan, and the former Soviet Union agreed to collaborate on Conceptual Design Activities for the International Thermonuclear Experimental Reactor (ITER). LLNL coordinated the initial U.S. technical effort. 


\section{rgy, Astrophysics, X-Ray Lasers}

- Development and analysis of the first heavy-ion fusion targets (both directly and indirectly driven) occurred with gain curves as function of intensity, range, and pulse energy.

- A definitive study of heavy-ion stopping in hot dense matter was published.

- Initial studies completed of heavy-ion beam propagation through the chamber, including such effects as space charge nonlinearities, filamentation, stripping, neutralization, photo-ionization, and target charging

- A chamber concept using granular wall material (CASCADE) with low chamber pressures developed.

1990s

- "Study of Recirculating Induction Accelerators as Drivers for Heavy Ion Fusion" published.

- First 3-D Particle in Cell Simulations (using the WARP code) produced of space charge dominated heavy-ion beams through acceleration, confinement and bending elements, and detailed comparison with several heavy-ion beam experiments, including the Lawrence Berkeley National Laboratory's (LBNL) 2-MV injector and MBE4.

- A design published of a distributed radiator target for inertial fusion, driven from two sides with heavy-ion beams (at a level of detail comparable to the designs used for the NIF).

- Publication of studies of intense heavy-ion beam transport through electric and magnetic quadrupoles in LLNL's 80-keV heavy-ion beam transport experiment, including the use of nonintercepting capacitive probe centroid measurements and detailed phase-space diagnostics using a gated-beam imager.

- Inertial Fusion Energy (IFE) power plant concept (HYLIFE-II) developed, using molten salt liquid-walls (FLiBe) and driven by a heavy-ion accelerator with high availability and low wall activation.

- A major systems code study (IBEAM) published systematizing the design of heavy-ion drivers.

- The formation of two national IFE organizations initiated: (1) the Heavy-Ion Fusion Virtual National Laboratory (HIF$\mathrm{VNL}$ ) which manages the heavy-ion fusion accelerator research at LLNL, LBNL, and the Princeton Plasma Physics Laboratory (PPPL); and (2) the IFE area of the Fusion Energy Virtual Laboratory of Technology, which coordinates all IFE technology research at the national laboratories and universities.

- LLNL researchers led a national effort to design the next Tokamak Plasma Experiment--a steady-state advanced tokamak that, if built, would have tested whether smaller, less expensive fusion reactors could be constructed.

- LLNL's Fusion Engineering International Experimental (FENIX) magnet facility tested, at 14 tesla, prototype ITER superconductors that can carry currents up to 46 kiloamperes.

- In a collaborative project with General Atomics (GA) and other researchers around the world, LLNL researchers using the DIII-D tokamak in San Diego, investigated the physics of tokamaks that incorporate a divertor. Also with GA, LLNL researchers established a Remote Experimental Site at LLNL to demonstrate offsite control of fusion experiments via fiber-optic transmission lines.

- In the Interaction of Magnetized Plasmas from Accelerated Compact Tori (IMPACT) experiments, LLNL researchers study shock-heated plasmas as a driver for IFE and as a potential fuel source for tokamaks.

\section{Inertial Confinement Fusion}

LLNL has also had a major, 30-year-program in the physics of inertial fusion targets, funded by Defense Programs as part of the Stockpile Stewardship Program in the NIF Programs Directorate. This work forms the necessary physics basis for Inertial Fusion Energy. Major highlights from this program are listed below in order to provide a more complete view of work on fusion at LLNL.

\section{0s}

- The first inertial confinement fusion (ICF) direct drive implosions and fusion neutrons produced on the JANUS Laser.

- First ICF radiation-driven implosions and fusion neutrons produced on the Cyclops Laser.

- The Shiva Laser completed, and 100 times liquid density compression of DT fuel demonstrated.

\section{0s}

- Improvements in coupling at $0.53 \mathrm{~nm}$ and $0.35 \mathrm{~nm}$ demonstrated on the Argus Laser that would be required for the success of the Nova target physics program.

- The Novette and Nova Lasers completed. Early experiments included achievement of adequate drive temperature, initial radiation symmetry control, and reduced hydrodynamic instability in the presence of ablation as required for ICF ignition and high gain with radiation-driven targets.

\section{0s}

- Completion of a wide range of experiments in the ICF Program on Nova developed the target physics basis for proceeding with the NIF.

- Beamlet constructed as a single-beam scientific prototype for the NIF.

- The NIF construction project initiated.

\section{0s}

- A program in high-average-power lasers for IFE initiated, using diode-pumped solid-state lasers with the 100-joule Mercury Laser as the technical focus. 
- On MTX, LLNL scientists and engineers injected pulsed microwave radiation of 2 billion watts into the plasma, demonstrating, for the first time, nonlinear absorption at the cyclotron resonance of the electrons.

- The Sustained Spheromak Physics Experiment was constructed to study energy confinement in the spheromak. The spheromak is a self-organized plasma, with many physics connections to magnetized plasmas in space and near the sun, and is potentially a good reactor configuration if confinement is good enough.

\section{0s}

- Magnetic Fusion Energy and Inertial Fusion Energy joined together to form the Fusion Energy Program.

- LLNL researchers participated in the successful demonstration of ITER magnets in Japan, together with the international community.

- Publication of results of experimental studies at LLNL of acceleration and bending (through 90 degrees) of a $80-\mathrm{kV}$, $\mathrm{K}+$ heavy-ion beam with implications for a recirculating induction accelerator.

- Commissioning of the 500-kV ion source and low-energy beam transport test stand at LLNL.

- Publication of a design of a close-coupled target for heavyion fusion.

\section{Highlights of Astrophysics in Physics at LLNL}

Kem Cook developed this timeline of achievements in astrophysics research in the PAT Directorate and predecessor organizations.

\section{0s}

- LLNL scientists discovered the basic mechanism of supernova explosions.

- LLNL researchers developed the numerical hydrodynamic calculations for general-relativistic collapse involved in the creation of a black hole.

- Astrophysical observations of a supernova remnant, an external galaxy, and the cosmic $x$-ray background radiation achieved using $x$-ray sounding rockets originally developed for test-ban treaty monitoring.

PAT scientists have often been in the forefront of astrophysical research. Almost a decade ago, seminal work on dark matter by LLNL's astrophysicists was featured in the October 14, 1993, issue of Nature. Work on the Massive Compact Halo Objects (MACHO) project was featured.

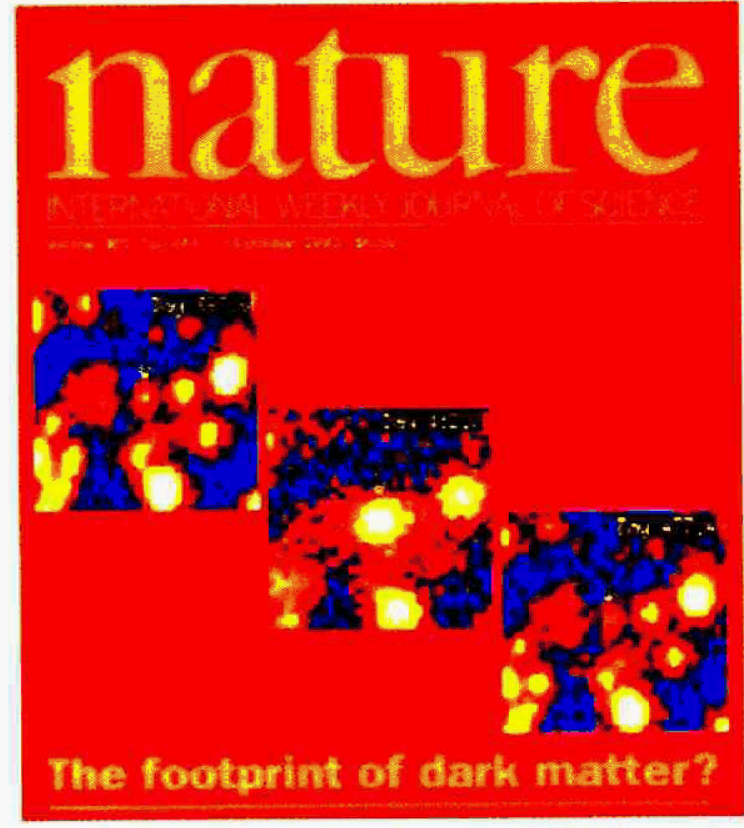

reprinted by permission from Nature (365:6447) copyrigh 1993 Macmillan Publishers I td. 1970s

- From modeling supernovae explosions, LLNL researchers developed accurate, general relativistic models describing the explosion mechanism in detail.

- The first hydrodynamic stellar evolution models were generated, and explorations of stellar evolution processes leading to supernovae explosions were begun.

- LLNL researchers predicted the existence of quark stars that were subsequently discovered in 2002.

\section{0s}

- LLNL researchers developed hydrodynamic supernova models. They explained element abundances and why the progenitor of SN 1987a, the nearest Type la supernova in modern times, was a blue supergiant star.

- An equation of state and an opacity code which become the standard for modeling stellar physics were developed, leading to a series of papers which are among the most cited in astrophysics.

1990s

- LLNL's Large Optics Diamond Turning Machine was used to figure (i.e., geometrically shape) the Keck Observatory infrared secondary mirror out of beryllium.

- LLNL led the development of astronomical adaptive optics systems and the laser guide star, resulting in the first functioning laser guide star system, and the construction of the Keck adaptive optics system that provides ground-based observations with greater spatial resolution than the Hubble Space Telescope.

- The Massive Compact Halo Objects (MACHO) collaboration among LLNL researchers and many university collaborators began, leading to the discovery of the first gravitational microlens and launching the field of microlensing surveys.

\section{$2000 s$}

- A large, LLNL-led team developed the first fully 3-D, massively parallel hydrodynamic stellar evolution and stellar structure code which is based upon LLNL-developed hydrodynamic codes.

\section{Highlights of X-ray Laser Research at LLNL}

Dennis Matthews, Jim Dunn, and Franz Weber developed this timeline of achievements in $x$-ray laser research at LLNL.

$X$-ray lasers are an invaluable tool for studying the expansion of high-density plasmas, particularly laser-produced plasmas, making them useful for LLNL's fusion and physics programs. This work was conducted in conjunction with personnel from the predecessor organization to the NIF Programs Directorate, i.e., the Laser Programs Directorate.

\section{2-1983}

- Early attempts to produce a resonantly photopumped x-ray laser using the Shiva and Novette Laser Facilities occurred.

\section{Friday the 13th, July 1984}

- The first laboratory $x$-ray laser, Ne-like Se, demonstrated at the Novette Laser Facility. 


\section{4-1985}

- The Nova 2 Beam Facility, especially designed for $x$-ray laser research, was constructed.

\section{5-1997}

- Continuous research led to further development and demonstration of $x$-ray lasers and their applications using the Nova 2 Beam Facility, including the following:

Ne-like Lasers up to Ar demonstrated, achieving lasing at $10 \mathrm{~nm}$

- Ni-like Lasers up to Au demonstrated, achieving lasing at $3.3 \mathrm{~nm}$.

- First multipass amplifier demonstrated.

- First x-ray laser hologram demonstrated.

- Dark field image of sperm cell and resolution of $50 \mathrm{~nm}$ achieved.

- X-ray laser probed plasmas demonstrated, including:

$\checkmark$ Hole boring in over- and under-dense plasmas

$\checkmark$ Radiography of laser imprinting in thin foils.

$X$-ray laser interferometry, Moiré deflectometry, demonstrated.

- High-efficiency $x$-ray lasers, leading to so-called tabletop designs, demonstrated.

\section{7-present}

- Research performed on high-efficiency, transient gain, tabletop $\mathrm{x}$-ray lasers at the Compact Multipulse Terawatt (COMET) Laser Facility in the PAT Directorate.

\section{June 3, 1997}

- Tabletop x-ray laser for Ne-like Ti at $32.5 \mathrm{~nm}$ observed at LLNL JANUS picosecond facility.

\section{8}

- COMET tabletop with multiple beams was constructed as a dedicated $x$-ray laser facility.

Gain saturation regime for Ni-like Pd at $14.7 \mathrm{~nm}$ demonstrated for the first time.

Pictured bottom right is the 2-beam Novette Laser, which was used to stimulate the soft $x$-ray lasing in the mid-1980s. The soft $x$-ray experiments were conducted inside the target chamber (in the background) using a 2,000-joule pulse of green light from each of the arms directed onto a small metal foil that was held in a postage-stamp-sized target. Although the Novette laser system served primarily as a test bed for advanced concepts of targets and lasers for the subsequent Nova system, its use led to the world's first demonstration of $x$-ray lasing. The experiments were undertaken in conjunction with researchers from the predecessor directorate to the current NIF Programs Directorate.

Shown on the cover of LLNL's Energy and Technology Review for November 1985 is a x-ray laser target. Irradiation with the two beams of the Novette Laser exploded and ionized the selenium foil in the center of the target assembly. This 1985 E\&TR issue was devoted to LLNL's work on laboratory $x$-ray lasing.
First qain measurements made on photo-pumped Ni-like Mo $4 \mathrm{f}-4 \mathrm{~d}$ line at $22.6 \mathrm{~nm}$, including:

0 First picosecond $x$-ray laser interferometry of plasmas (in collaboration with researchers from Colorado State University)

- Multistage injector-amplifier Ne-like $\mathrm{Cr}$ x-ray laser at $28.5 \mathrm{~nm}$ (in collaboration with researchers from the Multicharged lons Spectra Data Center in Moscow, Russia).

$\checkmark$ First demonstration of tabletop, picosecond laser-driven gas puff $\mathrm{x}$-ray laser for $\mathrm{Ne}$-like $\mathrm{Ar}$ at $46.9 \mathrm{~nm}$ (in collaboration with researchers from the Military University of Technology, Warsaw, Poland).

$\diamond$ First x-ray laser-induced photoelectron spectroscopy for material surface analysis (in collaboration with researchers in LLNL's Chemistry and Materials Science Directorate).

\section{9 - present}

- Inner-Shell Photo-lonization X-Ray Laser at $45 \AA$ developed, including the following:

An absorber/filter target package designed for high backside emission, 60-fs gain duration in lasant.

$10^{-4}$ conversion efficiency measured.

- Traveling wave pumping system in-line focus geometry suitable for ultrashort pulse laser systems ( $50 \mathrm{fs}$ ) designed.

Pump-Probe scheme to measure ultrafast rise time of $x$ ray source ( $50 \mathrm{fs}$ ) designed.

- Successful proposal,"X-ray Laser Based Imaging of Single Cells in Fully Hydrated State," submitted as part of National Science Foundation's Center For Biophotonics.
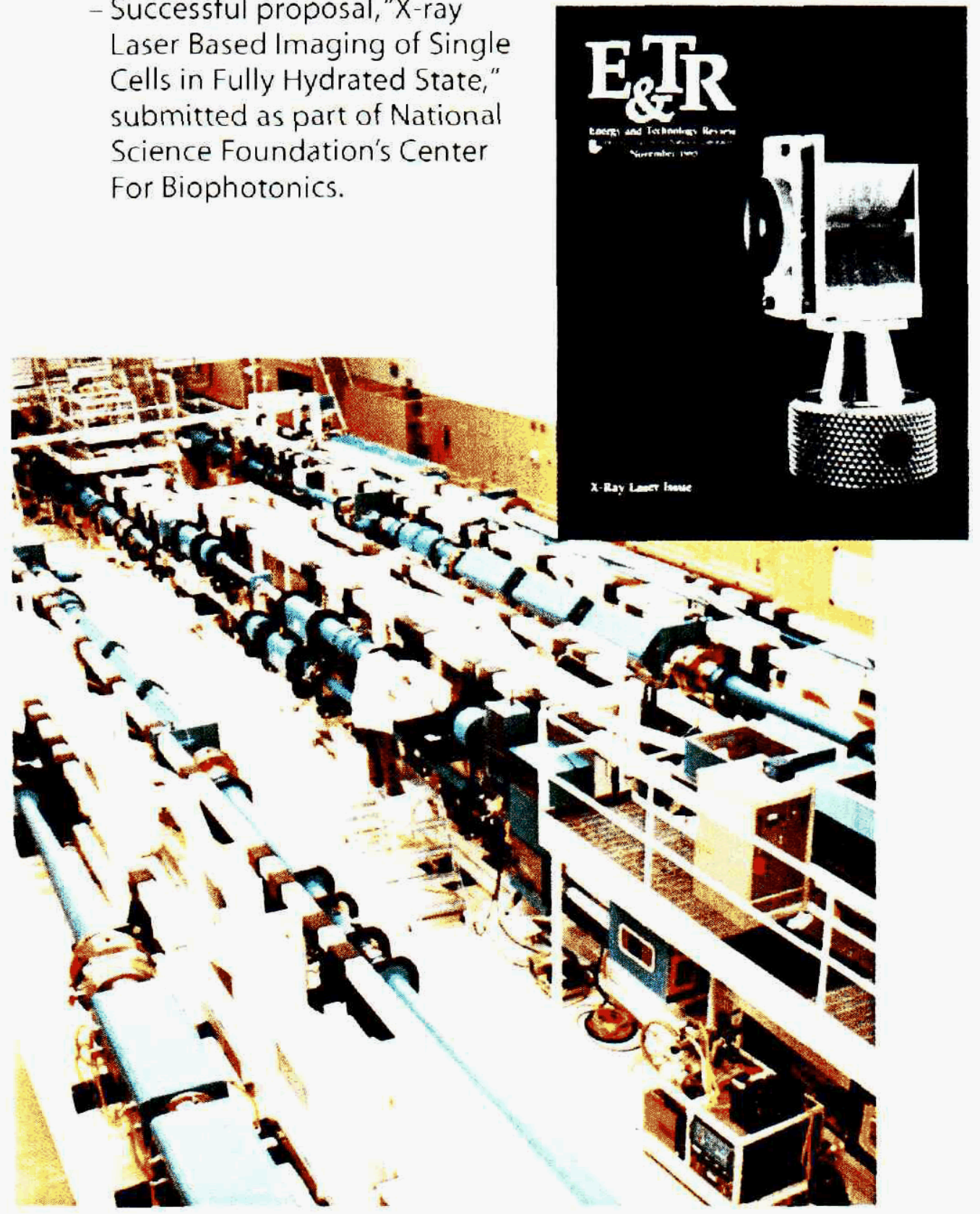


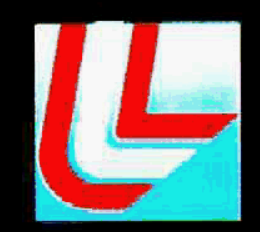

Lawrence Livermore National Laboratory PO Box 808,

Livermore, CA 94551

Visit the Laboratotory's

website at

http://www.llnl.gov
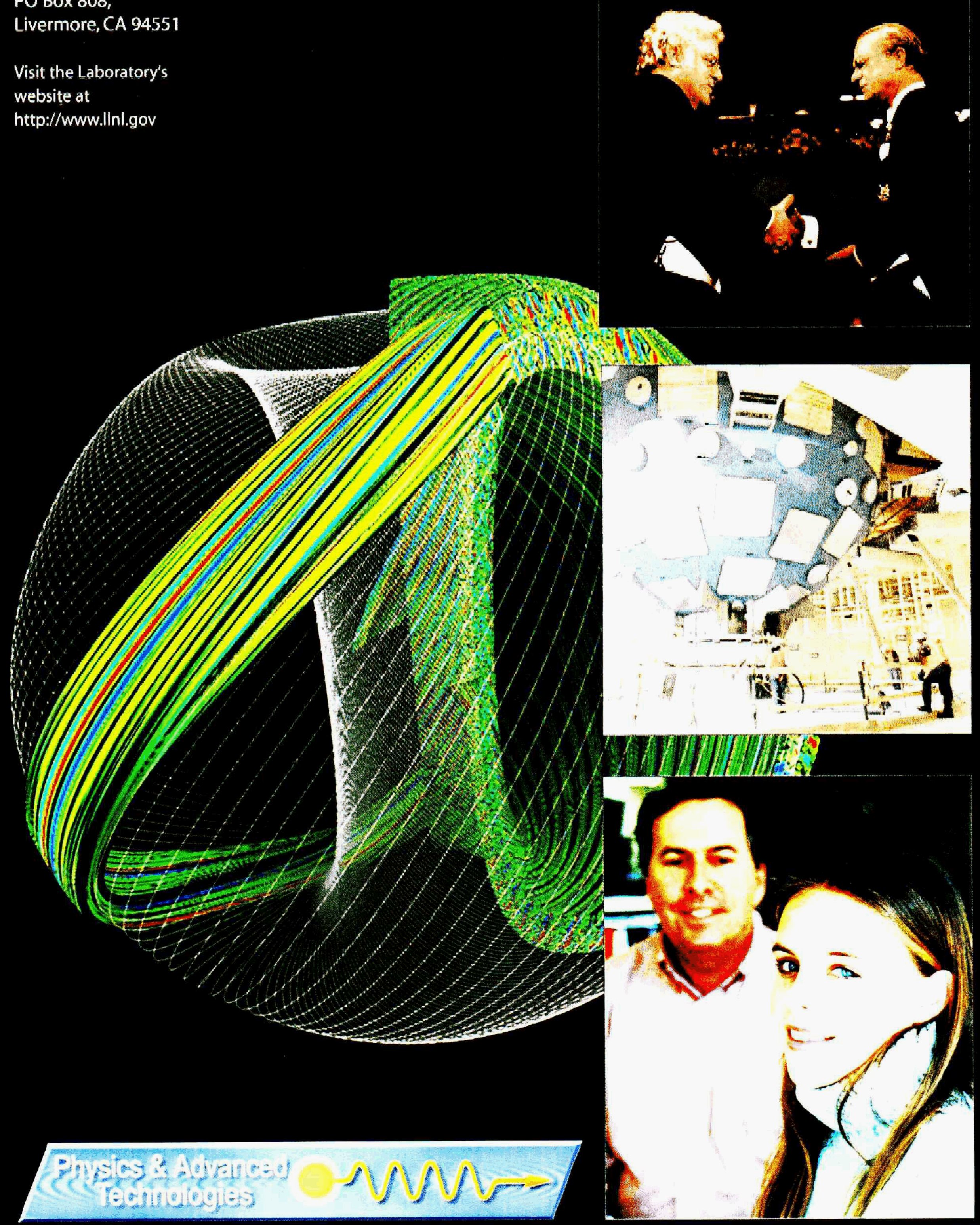